\title{
Decomposição de Grafos em Caminhos
}

Fábio Happ Botler

TESE APRESENTADA

$\mathrm{AO}$

Instituto De MatemáticA E EstatísticA

DA

UNIVERSIDADE DE SÃo PAUlO

PARA

OBTENÇÃO DO TÍTULO

$\mathrm{DE}$

DOUTOR EM CIÊNCIAS

\author{
Programa: Ciência da Computação \\ Orientadora: Prof. ${ }^{a}$ Dr. ${ }^{\text {a }}$ Yoshiko Wakabayashi
}

Durante o desenvolvimento deste trabalho o autor recebeu auxílio financeiro do CNPq e da FAPESP Proc. 2011/08033-0 e 2014/01460-8

São Paulo, janeiro de 2016 


\section{Decomposição de Grafos em Caminhos}

Esta é a versão original da tese elaborada pelo candidato Fábio Happ Botler, tal como submetida à Comissão Julgadora. 


\section{Resumo}

BOTLER, F. Decomposição de Grafos em Caminhos. 2016. 60 f. Tese (Doutorado) - Instituto de Matemática e Estatística, Universidade de São Paulo, São Paulo, 2016.

Uma decomposição de um grafo $G$ é um conjunto $\mathcal{D}=\left\{H_{1}, \cdots, H_{k}\right\}$ de subgrafos de $G$ dois-adois aresta-disjuntos que cobre o conjunto das arestas de $G$. Se $H_{i}$ é isomorfo a um grafo fixo $H$, para $1 \leq i \leq k$, então dizemos que $\mathcal{D}$ é uma $H$-decomposição de $G$. Neste trabalho, estudamos o caso em que $H$ é um caminho de comprimento fixo. Para isso, primeiramente decompomos o grafo dado em trilhas, e depois fazemos uso de um lema de desemaranhamento, que nos permite transformar essa decomposição em trilhas numa decomposição somente em caminhos. Com isso, obtemos resultados para três conjecturas sobre $H$-decomposição de grafos no caso em que $H=P_{\ell}$ é o caminho de comprimento $\ell$. Dois desses resultados resolvem versões fracas das Conjecturas de Kouider e Lonc (1999) e de Favaron, Genest e Kouider (2010), ambas para grafos regulares. Provamos que, para todo inteiro positivo $\ell$, (i) existe um inteiro positivo $m_{0}$ tal que se $G$ é um grafo $2 m \ell$-regular com $m \geq m_{0}$, então $G$ admite uma $P_{\ell}$-decomposição; (ii) se $\ell$ é ímpar, existe um inteiro positivo $m_{0}$ tal que se $G$ é um grafo $m \ell$-regular com $m \geq m_{0}$, e $G$ contém um $m$-fator, então $G$ admite uma $P_{\ell}$-decomposição. O terceiro resultado diz respeito a grafos altamente aresta-conexos: existe um inteiro positivo $k_{\ell}$ tal que se $G$ é um grafo $k_{\ell}$-aresta-conexo cujo número de arestas é divisível

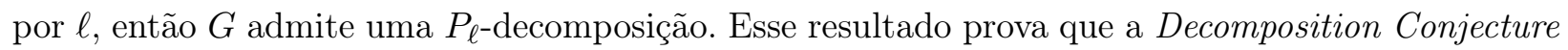
de Barát e Thomassen (2006), formulada para árvores, é verdadeira para caminhos.

Palavras-chave: grafo, decomposição em caminhos, alta aresta-conexidade, grafo regular. 


\section{Abstract}

Botler, F. Decomposition of Graphs into Paths. 2016. 60 f. Tese (Doutorado) - Instituto de Matemática e Estatística, Universidade de São Paulo, São Paulo, 2016.

A decomposition of a graph $G$ is a set $\mathcal{D}=\left\{H_{1}, \cdots, H_{k}\right\}$ of pairwise edge-disjoint subgraphs of $G$ that cover the set of edges of $G$. If $H_{i}$ is isomorphic to a fixed graph $H$, for $1 \leq i \leq k$, then we say that $\mathcal{D}$ is an $H$-decomposition of $G$. In this work, we study the case where $H$ is a path of fixed length. For that, we first decompose the given graph into trails, and then we use a disentangling lemma, that allows us to transform this decomposition into one consisting only of paths. With this approach, we tackle three conjectures on $H$-decomposition of graphs and obtain results for the case $H=P_{\ell}$ is the path of length $\ell$. Two of these results solve weakenings of a conjecture of Kouider and Lonc (1999) and a conjecture of Favaron, Genest and Kouider (2010), both for regular graphs. We prove that, for every positive integer $\ell$, (i) there is a positive integer $m_{0}$ such that, if $G$ is a $2 m \ell$-regular graph with $m \geq m_{0}$, then $G$ admits a $P_{\ell}$-decomposition; (ii) if $\ell$ is odd, there is a positive integer $m_{0}$ such that, if $G$ is an $m \ell$-regular graph with $m \geq m_{0}$ containing an $m$-factor, then $G$ admits a $P_{\ell}$-decomposition. The third result concerns highly edge-connected graphs: there is a positive integer $k_{\ell}$ such that if $G$ is a $k_{\ell}$-edge-connected graph whose number of edges is divisible by $\ell$, then $G$ admits a $P_{\ell}$-decomposition. This result verifies for paths the Decomposition Conjecture of Barát and Thomassen (2006), on trees.

Keywords: graph, path decomposition, highly edge-connected, regular graph. 


\section{Sumário}

Lista de Figuras $\quad$ vii

1 Introdução 1

2 Notação e conceitos básicos sobre grafos $\quad 5$

$\begin{array}{lll}3 & \text { Um lema de desemaranhamento } & 7\end{array}$

3.1 Trilhas, rastros, e sequências aumentantes . . . . . . . . . . . . . 8

3.2 Arestas pendentes e rastro-decomposições completas . . . . . . . . . . . . . . . 12

4 Decomposição de grafos regulares em caminhos de comprimento fixo 19

4.1 Decomposição de grafos regulares com cintura prescrita . . . . . . . . . . . . . 22

4.1 .1 Decomposições de grafos regulares . . . . . . . . . . . . . . . . 24

4.2 Grafos regulares ímpares com cintura pelo menos $\ell-1$ e grafos bipartidos . . . . . 26

4.2.1 Decomposições de grafos $m \ell$-regulares com cintura pelo menos $\ell-1 \ldots 26$

4.2 .2 Decomposições de grafos bipartidos . . . . . . . . . . . . . . . . . . . 29

5 Grafos altamente conexos $\quad 31$

5.1 Resultados preliminares . . . . . . . . . . . . . . . . . . . 32

5.1 .1 Splitting de vértices . . . . . . . . . . . . . . . . . . . 32

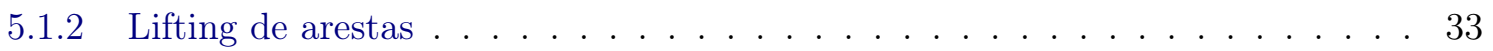

5.1 .3 Alta aresta-conexidade . . . . . . . . . . . . . . . . . . 34

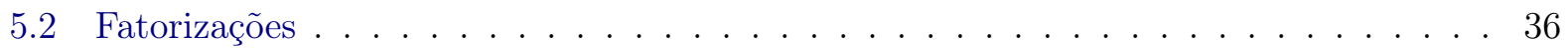

5.2 .1 Fatorizações fracionárias . . . . . . . . . . . . . . 36

5.2 .2 Bifatorizações . . . . . . . . . . . . . . . . . . . . . . 38

5.3 Decomposições de grafos bifatoráveis em $\ell$-caminhos . . . . . . . . . . . . . . 39

5.4 Decomposição de grafos altamente aresta-conexos em $\ell$-caminhos . . . . . . . . . . 43

6 Considerações finais $\quad 45$

$\begin{array}{ll}\text { Referências Bibliográficas } & 47\end{array}$ 


\section{Lista de Figuras}

3.1 Trilhas com comprimento 5 em grafos livres de triângulos. . . . . . . . . . . . 7

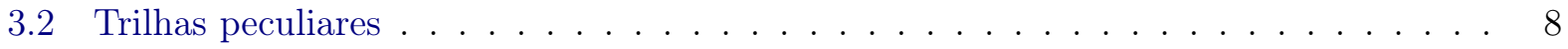

3.3 Sequência aumentante . . . . . . . . . . . . . . . . . . . . 10

3.4 Troca de arestas em sequências aumentantes perfeitas passo-a-passo . . . . . . . . . . 11

4.1 Teorema de Kotzig, e Bouchet e Fouquet. . . . . . . . . . . . . . . . . . . . . 19

4.2 Decomposições de um grafo 5 -regular. . . . . . . . . . . . . . . . . . . . 20

4.3 Grafo 5-regular que não admite $P_{5}$-decomposição. . . . . . . . . . . . . . . . . 20

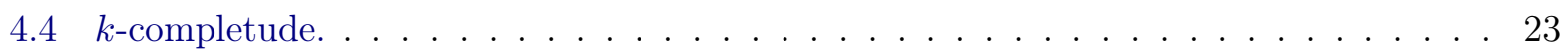

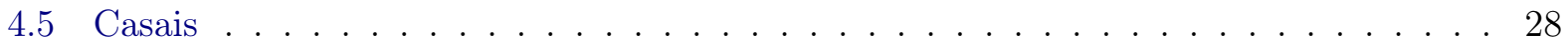

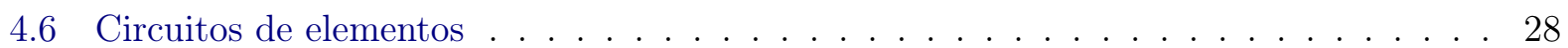

5.1 Exemplo de destacamento . . . . . . . . . . . . . . . . . . . 33

5.2 Exemplo de lifting . . . . . . . . . . . . . . . . . . . . . . . 33 


\section{Capítulo 1}

\section{Introdução}

Uma decomposição $\mathcal{D}=\left\{H_{1}, \ldots, H_{k}\right\}$ de um grafo $G$ é um conjunto de subgrafos arestadisjuntos de $G$ que cobre o conjunto das arestas de $G$. Uma implicação do Teorema de Hall (1935) diz que grafos regulares bipartidos admitem uma decomposição em emparelhamentos perfeitos. Uma consequência disso, já conhecida por Petersen em 1891, é que grafos regulares pares admitem uma decomposição em 2-fatores. Aqui, um grafo é dito par (resp. impar) se todos os seus vértices têm grau par (resp. ímpar).

Desde então, diversos resultados sobre decomposições surgiram na literatura. Por exemplo, Pyber [Pyb92] provou que todo grafo simples pode ser decomposto em no máximo quatro grafos ímpares. Jünger, Reinelt e Pulleyblank [JRP85] consideraram decomposições em subgrafos conexos com $k$ arestas (admitindo no máximo um subgrafo com menos que $k$ arestas), chamadas de $k$-partições. Eles provaram que todo grafo $k$-aresta-conexo admite uma $(k+1)$-partição, para $k=1,2,3$, e todo grafo 4 -aresta-conexo admite uma $s$-partição, para todo inteiro positivo $s$. Em particular, eles conjecturaram que grafos planares 2-aresta-conexos admitem 3-partições compostas apenas por trilhas.

Neste trabalho estamos interessados em decomposições $\mathcal{D}=\left\{H_{1}, \ldots, H_{k}\right\}$ de um grafo $G$ nas quais $H_{i}$ é isomorfo a um grafo fixo $H$, para todo $1 \leq i \leq k$. Tal decomposição é chamada de $H$ decomposição. Como observado por Häggkvist [Häg89], uma questão natural é, dados dois grafos $G$ e $H$, decidir quando $G$ admite uma $H$-decomposição.

"Given two graphs $G$ and $H$ and an inquisitive mind we may ask whether or not $G$ is the edge-disjoint union of copies of $H . "$ (HÄGGKVIST, 1989)

Uma condição obviamente necessária é que $|E(G)|$ seja divisível por $|E(H)|$. O resultado de Jünger, Reinelt e Pulleyblank enunciado acima afirma que se $G$ é conexo e tem um número par de arestas, então $G$ admite uma $P_{2}$-decomposição, onde $P_{2}$ é o caminho com duas arestas. Por outro lado, Dor e Tarsi [DT97] provaram que sempre que $H$ é conexo e contém pelo menos 3 arestas, o problema de decidir se $G$ admite uma $H$-decomposição é NP-completo. Assim, é natural procurar condições suficientes para obter tais decomposições. Para decomposições em triângulos, por exemplo, Nash-Williams [NW70] conjecturou que grau mínimo $3 n / 4$ seja suficiente, i.e, se $G$ é um grafo par com $n$ vértices e grau mínimo $\delta(G) \geq 3 n / 4$, (e $|E(G)|$ é divisível por 3), então $G$ admite uma $K_{3}$-decomposição. Com o objetivo de atacar a Conjectura de Nash-Williams, Barber, Kühn, Lo e Osthus [BKLO16] provaram que grafos pares com grau mínimo $\delta(G) \geq 9 n / 10+o(n)$ e tais que $|E(G)|$ é divisível por 3 admitem uma $K_{3}$-decomposição. 
No caso em que $H=T$ é uma árvore, Barát e Thomassen [BT06] conjecturaram que alta aresta-conexidade é suficiente, i.e, eles propuseram a seguinte conjectura.

Conjectura (Barát-Thomassen, 2006). Para cada árvore $T$, existe um inteiro positivo $k_{T}$ tal que, se $G$ é um grafo $k_{T}$-aresta-conexo e $|E(G)|$ é divisivel por $|E(T)|$, então $G$ admite uma T-decomposição.

A Conjectura de Barát e Thomassen, conhecida como Decomposition Conjecture, é o foco principal deste trabalho. Thomassen [Tho08a, Tho08b, Tho12, Tho13a, Tho13b] verificou a validade dessa conjectura para estrelas, algumas biestrelas, caminhos de comprimento 3, e caminhos de comprimento $2^{k}$, para todo inteiro positivo $k$. Neste trabalho, provamos que essa conjectura é verdadeira para caminhos de qualquer comprimento fixo.

Para atacar a Conjectura de Barát e Thomassen, investigamos primeiramente $T$-decomposições de grafos regulares. Em 1964, Ringel [Rin64] conjecturou que o grafo completo $K_{2 \ell+1}$ admite uma $T$-decomposição para qualquer árvore $T$ com $\ell$ arestas. A Conjectura de Ringel é comumente confundida com a Graceful Tree Conjecture que diz que toda árvore $T$ com $n$ vértices admite uma rotulação $f: V(T) \rightarrow\{0, \ldots, n-1\}$ tal que $\{1, \ldots, n-1\} \subseteq\{|f(x)-f(y)|: x y \in E(T)\}$. Uma vez que a Graceful Tree Conjecture implica a Conjectura de Ringel [Ros67], ambas são válidas para diversas classes, como estrelas, caminhos, biestrelas, lagartas e lagostas (veja [Gol72, EH06]). Häggkvist [Häg89] generalizou a Conjectura de Ringel para grafos regulares.

Conjectura 1.1 (Graham-Häggkvist, 1989). Para cada árvore $T$ com $\ell$ arestas, se $G$ é um grafo

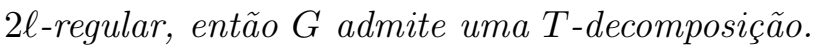

Em particular, Häggkvist [Häg89] provou que a Conjectura 1.1 vale quando a cintura de $G$ é maior ou igual ao diâmetro de $T$. Para mais resultados sobre decomposições de grafos regulares em árvores, veja [Fin90, JTT91, Sne91, JKW13, Erd14]. No caso particular em que $H=P_{\ell}$ é o caminho com $\ell$ arestas e $G$ é um grafo regular, Kouider e Lonc [KL99] melhoram o resultado de Häggkvist, provando que grafos $2 \ell$-regulares $G$ com cintura $g \geq(\ell+3) / 2$ admitem uma $P_{\ell}$-decomposição $\mathcal{D}$ tal que cada vértice de $G$ é vértice final de exatamente dois caminhos de $\mathcal{D}$. Eles também conjecturaram que esse fato deve valer para todo grafo $2 \ell$-regular, i.e, que todo grafo $2 \ell$-regular $G$ admite uma

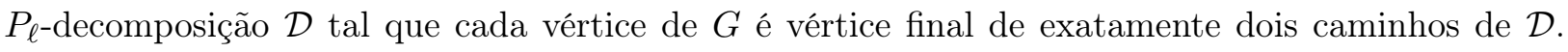
No Capítulo 4, provamos uma versão mais fraca da Conjectura de Kouider e Lonc. Nós provamos que, para cada $\ell$ e $g$ inteiros positivos tais que $g \geq 3$, existe $m_{0}=m_{0}(\ell, g)$ tal que, se $G$ é um grafo

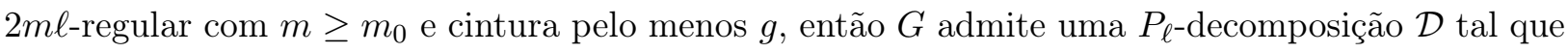
cada vértice de $G$ é vértice final de exatamente $2 m$ caminhos de $\mathcal{D}$.

Um outro resultado relacionado aos resultados citados acima é devido a Kotzig [Kot57], que provou que um grafo 3-regular $G$ admite uma $P_{3}$-decomposição se e somente se $G$ contém um emparelhamento perfeito. Favaron, Genest, e Kouider [FGK10] conjecturaram que esse resultado pode ser generalizado, i.e, que grafos $\ell$-regulares ímpares que contêm um emparelhamento perfeito admitem $P_{\ell^{-}}$decomposições. Nesse caso diminuímos o grau dos vértices do grafo pela metade, porém introduzimos um emparelhamento perfeito. No Capítulo 4, provamos uma versão mais fraca da Conjectura de Favaron, Genest, e Kouider. Provamos que, para cada $\ell$ e $g$ inteiros positivos tais que $\ell$ é ímpar e $g \geq 3$, existe $m_{0}=m_{0}(\ell, g)$ tal que, se $G$ é um grafo $m \ell$-regular com $m \geq m_{0}$,

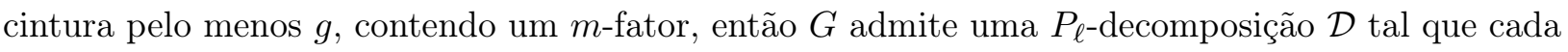


vértice de $G$ é vértice final de exatamente $m$ caminhos de $\mathcal{D}$. Por fim, como sugerido por Häggkvist, conjecturamos que esse fato deve valer de forma mais geral, com $m_{0}=1$.

"It is a trend in modern mathematics that if you can not solve a particular problem you can at least formulate a more general one, thus increasing the amount of frustration in the world and keeping your collegues on their toes." (HÄGGKVIST, 1989)

A ferramenta central desenvolvida neste trabalho é um lema de desemaranhamento (veja Capítulo 3), que se mostrou útil para atacar tanto as Conjecturas de Graham e Häggkvist, e de Favaron, Genest, e Kouider no Capítulo 4, quanto a Conjectura de Barát e Thomassen no Capítulo 5. Outro conceito importante é o de decomposição completa, introduzido primeiramente em [BMW15], e que será usado frequentemente neste trabalho. Grosseiramente, aqui a completude (de uma decomposição) representa um conjunto especial de propriedades de uma decomposição. Ao longo de nossas provas, desenvolvemos um algoritmo que recebe uma decomposição completa $\mathcal{D}$ e devolve uma decomposição completa "melhor" $\mathcal{D}^{\prime}$ (no sentido de $\mathcal{D}^{\prime}$ estar mais próxima da decomposição desejada), que mantém a propriedade de completude. Dessa forma, dado um grafo $G$ construímos inicialmente uma decomposição $\mathcal{D}$ para $G$, provamos que $\mathcal{D}$ é uma decomposição completa, e usamos o algoritmo para obter a decomposição desejada.

No Capítulo 2, apresentamos brevemente os conceitos sobre grafos que são mais relevantes neste trabalho. Nos capítulos seguintes reorganizamos os resultados em [BMOWb, BMOWc] como segue. No Capítulo 3, provamos um lema de desemaranhamento (Lema 3.9), que permite que, sob algumas condições, troquemos arestas entre elementos de uma decomposição de um grafo $G$ em trilhas para obter uma nova decomposição de $G$ em caminhos. No Capítulo 4, usamos o Lema 3.9 para obter decomposições em caminhos de comprimento fixo de uma grande família de grafos regulares. No Capítulo 5, generalizamos uma parte da técnica desenvolvida por Thomassen que lida com decomposições de grafos altamente aresta-conexos. Essa técnica nos dá a estrutura necessária para aplicarmos alguns lemas obtidos no Capítulo 4, e provar a Conjectura de Barát e Thomassen para caminhos de qualquer comprimento fixo. 


\section{Capítulo 2}

\section{Notação e conceitos básicos sobre grafos}

A terminologia básica e a notação usadas neste trabalho são padrões [Bol98, Die10]. Um multigrafo é um par $G=(V, E)$. Os conjuntos $V$ e $E$, também denotados por $V(G)$ e $E(G)$, são, respectivamente, os conjuntos dos vértices e das arestas de $G$. Um multigrafo é dito simples ou, simplesmente, um grafo se não contém arestas paralelas. Todos os grafos e multigrafos considerados aqui são finitos e não têm laços. Neste trabalho utilizamos predominantemente grafos, exceto apenas no Capítulo 5, mais especificamente, na prova do Lemma 5.17. Assim, as seguintes definições são apresentadas para grafos, mas podem ser facilmente estendidas para multigrafos.

Denotamos por $d_{G}(v)$ o grau de um vértice $v \in V$ e, quando $G$ é claro pelo contexto, escrevemos apenas $d(v)$. Se $d(v)=0$, então dizemos que $v$ é um vértice isolado em $G$. Se $d(v)=r$ para todo vértice $v$ de $G$, então dizemos que $G$ é $r$-regular. Dizemos que um grafo $H$ é um subgrafo de $G$ se $V(H) \subseteq V(G)$ e $E(H) \subseteq E(G)$, e usamos frequentemente os operadores aritméticos + e - da seguinte forma. Se $F$ é um conjunto de arestas, e $e$ é uma aresta, então denotamos por $F+e$ e $F-e$ os conjuntos $F \cup\{e\}$ e $F \backslash\{e\}$, respectivamente. Se $H$ é um subgrafo de $G$, e $e$ é uma aresta de $G$, então denotamos por $H+e$ o grafo obtido de $(V(H), E(H)+e)$ pela adição dos vértices de $e$ que não estão presentes em $V(H)$; e por $H-e$ o grafo obtido de $(V(H), E(H)-e)$ pela remoção dos vértices incidentes a $e$ que ficaram isolados. Dado $F \subseteq E$, denotamos por $G[F]$ o subgrafo de $G$ induzido por $F$, i.e, o grafo obtido de $(V(G), F)$ pela remoção de seus vértices isolados. Também denotamos por $d_{F}(v)$ o número de arestas em $F$ que são incidentes a $v$ ou, equivalentemente, o grau de $v$ em $G[F]$.

Seja $G=(V, E)$ um grafo. Uma trilha em $G$ é um subgrafo de $G$ que admite uma sequência de vértices $T=v_{0} v_{1} \cdots v_{\ell}$ tal que $v_{i} v_{i+1} \in E$, para $0 \leq i \leq \ell-1$, e $v_{i} v_{i+1} \neq v_{j} v_{j+1}$, para $0 \leq i<j \leq \ell-1$. Também é conveniente nos referirmos a uma trilha $T=v_{0} v_{1} \cdots v_{\ell}$ como o subgrafo de $G$ induzido pelas arestas $v_{i} v_{i+1}$ para $i=0, \ldots, \ell-1$. O comprimento de uma trilha é sua quantidade de arestas. Também dizemos que uma trilha de comprimento $\ell$ é uma $\ell$-trilha. Um caminho em $G$ é uma trilha que admite uma sequência $P=v_{0} v_{1} \cdots v_{\ell}$ tal que $v_{i} \neq v_{j}$, para $0 \leq i<j \leq \ell$. Dizemos também que $v_{0}$ e $v_{\ell}$ são os vértices finais de $P$, e que $P$ liga $v_{0}$ a $v_{\ell}$. Um circuito é uma trilha que admite uma sequência $C=v_{0} v_{1} \cdots v_{\ell-1} v_{0}$ tal que $v_{i} \neq v_{j}$, para $0 \leq i<j \leq \ell-1$. O caminho de comprimento $\ell$, também chamado de $\ell$-caminho, é denotado por $P_{\ell}$. Dados dois vértices, $u$ e $v$ em $G$, a distância entre $u$ e $v$ é o comprimento de um caminho mais curto ligando $u$ e $v$ em $G$; o diâmetro de $G$ é a maior distância entre dois vértices de $G$; e a cintura de $G$ é o comprimento de um circuito mais curto em $G$.

Uma orientação $O$ de um subconjunto $F \subseteq E$, é uma atribuição de uma direção (de um vértice 
para o outro) a cada aresta em $F$. Se uma aresta $e=u v$ em $F$ é direcionada de $u$ para $v$, dizemos que $e$ sai de $u$ e entra em $v$. Dado um vértice $v$ de $G$, denotamos por $d_{O}^{+}(v)$ (resp. $d_{O}^{-}(v)$ ) o número de arestas de $F$ que saem de (resp. entram em) $v$ com respeito a $O$. Neste trabalho usamos o termo grafo Euleriano como sinônimo de grafo par, i.e, um grafo Euleriano é um grafo que contém apenas vértices de grau par. Em outras palavras, aqui permitimos que um grafo Euleriano seja desconexo, e evitamos usar o termo par para se referir a grafos. Uma orientação Euleriana de um grafo Euleriano $G$ é uma orientação $O$ de $E(G)$ tal que $d_{O}^{+}(v)=d_{O}^{-}(v)$ para todo vértice $v$ em $V$. Além disso, dizemos que um subconjunto $F \subseteq E$ é Euleriano se $G[F]$ é Euleriano. Denotamos por $G=(A, B ; E)$ um grafo bipartido $G$ com bipartição $A, B$ do seu conjunto de vértices.

Dizemos que um conjunto $\left\{H_{1}, \ldots, H_{k}\right\}$ de subgrafos de $G$ é uma decomposição de $G$ se $\bigcup_{i=1}^{k} E\left(H_{i}\right)=E$ e $E\left(H_{i}\right) \cap E\left(H_{j}\right)=\emptyset$ para todo $1 \leq i<j \leq k$. Seja $\mathcal{H}$ uma família de grafos. Uma $\mathcal{H}$-decomposição $\mathcal{D}$ de $G$ é uma decomposição de $G$ tal que cada elemento de $\mathcal{D}$ é isomorfo a um elemento de $\mathcal{H}$. Além disso, se $\mathcal{H}=\{H\}$, então dizemos que $\mathcal{D}$ é uma $H$-decomposição.

Um subgrafo $H$ de $G$ é dito gerador ou fator de $G$ se $V(H)=V(G)$. Uma fatorização de um grafo $G$ é uma decomposição de $G$ em fatores. Um $k$-fator $H$ é um fator $k$-regular de $G$, e uma $k$-fatorização de $G$ é uma decomposição de $G$ em $k$-fatores. O seguinte resultado, o clássico Teorema de Fatorização de Petersen [Pet91], é essencial para os passos indutivos em nossas provas, e é usado frequentemente.

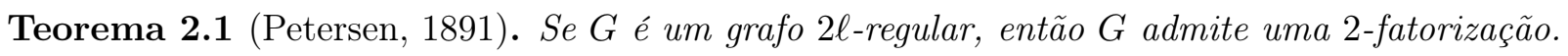

Seja $G$ um grafo conexo. A aresta-conexidade de $G$ é o menor inteiro $k$ tal que existe um conjunto $F \subseteq E(G)$ com $|F|=k$ e tal que $G-F$ é desconexo. Se $G$ tem aresta-conexidade $k$, então $G$ é dito $k$-aresta-conexo. O célebre Teorema de Menger [Men27] diz que dado um inteiro positivo $k$, um grafo $G$ é $k$-aresta-conexo se e somente se para todo par de vértices $u$ e $v$ existem pelo menos $k$ caminhos dois-a-dois aresta-disjuntos ligando $u$ a $v$ em $G$.

Os demais conceitos que serão utilizados neste trabalho serão apresentados ao longo do texto. No Capítulo 3 definimos rastros, que são utilizados para fixar ordens específicas dos vértices das trilhas de uma decomposição. No Capítulo 5 definimos fatorizações fracionárias e bifatorizações que estendem o conceito de fatorização para lidar convenientemente com grafos bipartidos altamente aresta-conexos. 


\section{Capítulo 3}

\section{Um lema de desemaranhamento}

Para decompor um grafo $G$ em cópias de um dado grafo $H$, uma estratégia consiste em obter num primeiro passo uma decomposição de $G$ em elementos que se assemelham a $H$. Por exemplo, para decompor um grafo em caminhos de comprimento $\ell$, podemos primeiramente encontrar uma decomposição $\mathcal{D}$ em trilhas de comprimento $\ell$. Se as trilhas em $\mathcal{D}$ não contêm circuitos, então $\mathcal{D}$ é uma decomposição em caminhos. Isso ocorre, por exemplo, no caso de grafos com cintura alta, mais especificamente, cintura maior que $\ell$. Se em $\mathcal{D}$ há trilhas que não são caminhos, usamos técnicas de desemaranhamento, isso é, procedimentos de trocas de arestas entre os elementos de $\mathcal{D}$, com o objetivo de encontrar uma nova decomposição $\mathcal{D}^{\prime}$ com menos elementos que contenham circuitos.

Em [HLY99], Heinrich, Liu, e Yu mostram como decompor grafos $3 m$-regulares que contêm um $m$-fator em caminhos de comprimento 3. Para isso, eles constroem uma decomposição do grafo em trilhas com 3 arestas, i.e, 3-caminhos e triângulos, e depois mostram que se nessa decomposição existe algum triângulo $X$, então existe um outro elemento $Y$ tal que $X \cup Y$ pode ser decomposto em dois caminhos de comprimento 3. Em [BMW15], decompomos um grafo 5-regular, que contém um emparelhamento perfeito e é livre de triângulos, em trilhas de comprimento 5 que podem conter um $C_{4}$ (veja Figura 3.1). Depois mostramos que se trocarmos arestas entre os elementos dessa decomposição numa determinada ordem, obtemos uma decomposição em 5-caminhos. Em [KL99], Kouider e Lonc decompõem grafos $2 \ell$-regulares com cintura pelo menos $(\ell+3) / 2$ em trilhas de comprimento $\ell$, e mostram que para cada trilha $X_{0}$ que não é um caminho existe uma sequência $X_{0} X_{1} \cdots X_{k}$ de elementos dessa decomposição tal que é possível trocar uma aresta de $X_{i} \operatorname{com} X_{i+1}$ $(i=0, \ldots, k-1)$ e obter apenas caminhos de comprimento $\ell$.
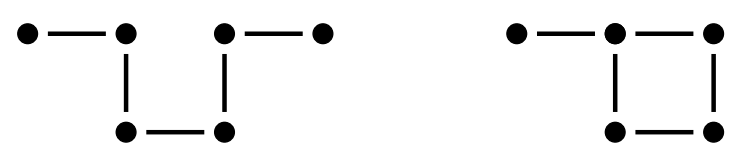

Figura 3.1: Trilhas com comprimento 5 em grafos livres de triângulos.

Neste capítulo apresentamos uma técnica de desemaranhamento que desenvolvemos [BMOWb] para caminhos de qualquer comprimento fixo. Mais precisamente, provamos um resultado, Lema 3.9, que garante que, dada uma decomposição de um grafo em certas trilhas especiais, é possível trocar arestas dos elementos dessa decomposição e construir uma decomposição com mais caminhos (que preserva algumas propriedades). Nossa técnica se assemelha à técnica introduzida por Kouider e Lonc [KL99], entretanto aqui fazemos uso de um grau mínimo suficientemente alto para compensar a exigência de cintura alta. 


\subsection{Trilhas, rastros, e sequências aumentantes}

Lembramos que uma trilha é um grafo $T$ para o qual existe uma sequência $B=x_{0} \cdots x_{\ell}$ de seus vértices (possivelmente com repetições) tal que $E(T)=\left\{x_{i} x_{i+1}: 0 \leq i \leq \ell-1\right\}$ e $x_{i} x_{i+1} \neq x_{j} x_{j+1}$, para $i \neq j$. Chamamos tal sequência de vértices $B$ de rastro de $T$, e dizemos que $T$ é a trilha induzida pelo rastro $B$. Os vértices $x_{0}$ e $x_{\ell}$ são chamados de vértices finais de $B$. Note que um caminho admite apenas dois possíveis rastros, enquanto um circuito de comprimento $\ell$ admite $2 \ell$ rastros.

Dado um rastro $B=x_{0} \cdots x_{\ell}$, denotamos por $B^{-}$o rastro $x_{\ell} \cdots x_{0}$, e, para facilitar a notação, denotamos por $V(B)$ e $E(B)$ os conjuntos $\left\{x_{0}, \ldots, x_{\ell}\right\}$ de vértices e $\left\{x_{i} x_{i+1}: 0 \leq i \leq \ell-1\right\}$ de arestas de $B$, respectivamente. Além disso, denotamos por $\bar{B}$ a trilha $(V(B), E(B))$. Será conveniente dizer que um rastro $B=x_{0} \cdots x_{\ell}$ visita os vértices $x_{0}, \ldots, x_{\ell}$ e as arestas $x_{0} x_{1}, \ldots, x_{\ell-1} x_{\ell}$ (nessas ordens), e que $x_{0} x_{1}$ é a aresta inicial de $B$ e $x_{\ell-1} x_{\ell}$ é a aresta final de $B$, ou que $B$ começa com $x_{0} x_{1}$ e acaba com $x_{\ell-1} x_{\ell}$. Um conjunto $\mathcal{B}$ de rastros aresta-disjuntos de um grafo $G$ é uma rastro-decomposição (ou decomposição em rastros) de $G$ se $\bigcup_{B \in \mathcal{B}} E(B)=E(G$ ). Note que o conjunto $\overline{\mathcal{B}}=\{\bar{B}: B \in \mathcal{B}\}$, chamado de decomposição subjacente a $\mathcal{B}$, é uma decomposição de $G$ em trilhas. Notamos também que se $B_{i}$ e $B_{j}$ são rastros de uma rastro-decomposição $\mathcal{B}$ tal que $E\left(B_{i}\right) \cap E\left(B_{j}\right) \neq \emptyset$, então $B_{i}=B_{j}$ e, consequentemente, $\bar{B}_{i}=\bar{B}_{j}$ (isso é, $B_{i}$ e $B_{j}$ induzem a mesma trilha).

Dizemos que um rastro $x_{0} x_{1} \cdots x_{\ell}$ é peculiar se o rastro $x_{1} \cdots x_{\ell-1}$ induz um caminho em $G$, e uma trilha $T$ é peculiar se existe um rastro peculiar de $T$ (veja Figura 3.2). Se um rastro peculiar (resp. trilha peculiar) contém $\ell$ arestas, então dizemos que ele é um $\ell$-rastro peculiar (resp. $\ell$ trilha peculiar). Aqui fixamos o objeto central deste trabalho: uma rastro-decomposição $\mathcal{B}$ é dita uma $\ell$-decomposição se todo elemento de $\mathcal{B}$ é um $\ell$-rastro peculiar. Note que não carregamos os termos "rastro" ou "peculiar" em $\ell$-decomposições, i.e, neste texto, todos os elementos de uma $\ell$-decomposição são rastros peculiares. Se a decomposição subjacente a $\mathcal{B}$ é uma decomposição em caminhos de $G$, então dizemos que $\mathcal{B}$ é uma $\ell$-decomposição em caminhos. Podemos omitir o comprimento $\ell$, quando este for claro no contexto.

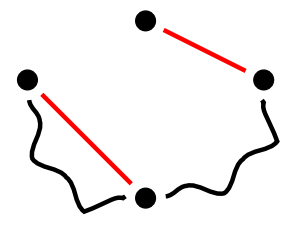

(a)

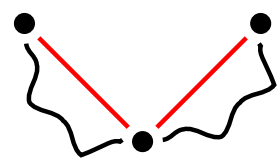

(d)

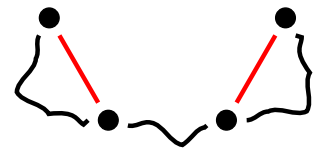

(b)

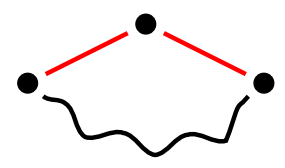

(e)

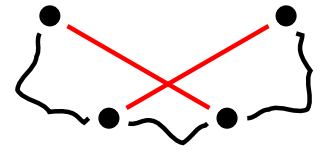

(c)

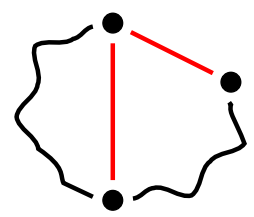

(f)

Figura 3.2: Exemplos de trilhas peculiares. As arestas vermelhas são iniciais e finais.

Suponha que exista um conjunto $F$ de arestas de um grafo $G$ tal que $G-F$ admite uma decomposição $\mathcal{D}^{\prime}$ em caminhos. Suponha também que possamos estender cada caminho de $\mathcal{D}^{\prime}$ com 
uma aresta de $F$ em cada um de seus vértices finais, e de forma que cada aresta em $F$ seja usada exatamente uma vez. Neste caso, cada caminho de $\mathcal{D}^{\prime}$ dá origem a uma trilha peculiar. Em particular, podemos obter uma rastro-decomposição $\mathcal{B}$ de $G$. Agora fixe um vértice $v$ de $G$. O número $h(v)$ de rastros de $\mathcal{B}$ que acabam imediatamente após visitar $v$ é exatamente o número de trilhas de $\mathcal{D}^{\prime}$ que têm $v$ como vértice final. Quando $h(v)$ é grande com respeito ao comprimento máximo de um rastro em $\mathcal{B}$ algumas propriedades surgem. A definição seguinte apresenta uma tal propriedade central nesta seção.

Definição 3.1 (Viabilidade). Seja $\ell$ um inteiro positivo. Seja $G$ um grafo e $\mathcal{B}$ uma $\ell$-decomposição de $G$. Dizemos que $\mathcal{B}$ é viável se para todo $v \in V(G)$ vale o seguinte: se $T$ é uma $\ell$-trilha de $G$ (não necessariamente admitindo rastro em $\mathcal{B}$ ) e $v$ está contido em um circuito de $T$, então existe um elemento $B$ em $\mathcal{B}$ tal que $B$ contém uma aresta vu onde u é vértice final de $B$ e $u \notin V(T)$.

O Lema 4.6 apresenta uma condição suficiente para a viabilidade de $\ell$-decomposições de grafos com restrições na cintura. O Lema 4.15 lida com o caso especial em que o grafo é bipartido.

Para cada rastro peculiar $B$ em $G$, seja $\tau(B)$ o número de vértices finais de $B$ com grau maior que 1 , e seja $\mathcal{B}$ uma $\ell$-decomposição viável de $G$ que minimiza $\tau(\mathcal{B})=\sum_{B \in \mathcal{B}} \tau(B)$ (na Figura 3.2a, temos que $\tau(B)=1$, e nas Figuras 3.2b-f, temos que $\tau(B)=2$ ). Se $\tau(\mathcal{B})=0$, então $\mathcal{B}$ é uma $\ell$-decomposição em caminhos. Portanto, suponha que $\tau(\mathcal{B})>0$. Uma vez que $\tau(\mathcal{B})>0$, existe um rastro peculiar $B_{0}$ em $\mathcal{B}$ que não é um caminho. Seja $x$ um vértice final de $B_{0}$ de grau maior que 1 em $\bar{B}_{0}$, e seja $C$ um circuito em $\bar{B}_{0}$ que contém $x$. Considere um vizinho $v$ de $x$ em $C$. Como $\mathcal{B}$ é viável, existe um elemento $B_{1}$ em $\mathcal{B}$ que contém a aresta $v u$, tal que $u \notin V\left(B_{0}\right)$ e $u$ é um vértice final de $B_{1}$. Agora, sejam $B_{0}^{\prime}$ e $B_{1}^{\prime}$ tais que $\bar{B}_{0}^{\prime}=\bar{B}_{0}-v x+v u, \bar{B}_{1}^{\prime}=\bar{B}_{1}-v u+v x$, e seja $\mathcal{B}^{\prime}=\mathcal{B}-B_{0}-B_{1}+B_{0}^{\prime}+B_{1}^{\prime}$. Temos que $\tau\left(B_{0}^{\prime}\right)=\tau\left(B_{0}\right)-1$. Suponha que a decomposição obtida $\mathcal{B}^{\prime}$ seja viável. Se $\tau\left(B_{1}^{\prime}\right) \leq \tau\left(B_{1}\right)$, então $\mathcal{B}^{\prime}$ é uma $\ell$-decomposição de $G$ tal que $\tau\left(\mathcal{B}^{\prime}\right)=$ $\sum_{B \in \mathcal{B}^{\prime}} \tau(B)<\sum_{B \in \mathcal{B}} \tau(B)=\tau(\mathcal{B})$, uma contradição à minimalidade de $\tau(\mathcal{B})$. Logo, temos que $\tau\left(B_{1}^{\prime}\right)=\tau\left(B_{1}\right)+1$ e $\bar{B}_{1}^{\prime}$ contém um circuito $C^{\prime}$ que contém $x v$. Agora, consideramos um vizinho $v^{\prime}$ de $x$ em $C^{\prime}$ tal que $v^{\prime} \neq v$, e repetimos essa operação enquanto necessário considerando $B_{1}^{\prime}$ e $v^{\prime}$ em vez de $B_{0}$ e $v$. Nós mostramos nesta seção que, sob algumas premissas, após repetir essa operação um número finito de vezes, obtemos uma decomposição melhor (uma $\ell$-decomposição com mais rastros induzindo caminhos do que a anterior). A Definição 3.2 formaliza quais propriedades a sequência de trilhas deve satisfazer para garantir essa melhora.

Seja $\mathcal{B}$ uma $\ell$-decomposição de um grafo $G$, e seja $S=B_{1} B_{2} \cdots B_{r}$ uma sequência de rastros (não necessariamente distintos) de $G$, onde $B_{i}=b_{0}^{i} b_{1}^{i} \cdots b_{\ell}^{i}$, para $i=1, \ldots, r$. Dizemos que $S$ é uma $\mathcal{B}$-sequência se $B_{i} \in \mathcal{B}$ ou $B_{i}^{-} \in \mathcal{B}$, para $i=1, \ldots, r$. O comprimento de uma $\mathcal{B}$-sequência $S$ é o número $r$ de rastros em $S$. No que segue, estamos interessados em tais $\mathcal{B}$-sequências $S=B_{1} B_{2} \cdots B_{r}$, onde o vértice $b_{0}^{1}$, o primeiro vértice de $B_{1}$, desempenha um papel importante. Exigimos que cada elemento $B_{i}$ de $S$, exceto o último (o rastro $B_{r}$ ), contenha o vértice $b_{0}^{1}$. Denotamos por $s(i)$ o menor indice positivo tal que $b_{s(i)}^{i}=b_{0}^{1}$. Como precisamos nos referir frequentemente ao vértice $b_{s(i)-1}^{i}(\mathrm{o}$ vértice de $B_{i}$ que é visitado antes de $b_{0}^{1}$ ) e ao vértice $b_{s(i)+1}^{i}$ (o vértice de $B_{i}$ que é visitado depois de $b_{0}^{1}$ ), para facilitar a notação, nós também os denotamos por $b_{*}^{i}$ e $b_{+}^{i}$ (isso é, $b_{*}^{i}:=b_{s(i)-1}^{i}$ e $\left.b_{+}^{i}:=b_{s(i)+1}^{i}\right)$, respectivamente. Note que mesmo que $\bar{B}_{i}=\bar{B}_{j}$, nós podemos ter $s(i) \neq s(j)$, porém, nesse caso, temos $b_{*}^{i}=b_{+}^{j}$ e $b_{+}^{i}=b_{-}^{j}$. Nesse contexto, também nomeamos duas arestas especiais de cada $B_{i}$; as arestas $e_{i}:=b_{*}^{i} b_{0}^{1}$ (a aresta visitada por $B_{i}$ "para entrar" em $b_{0}^{1}$ ), e $f_{i}:=b_{0}^{i} b_{1}^{i}$ (a aresta inicial de $\left.B_{i}\right)$. Veja a Figura 3.3. 


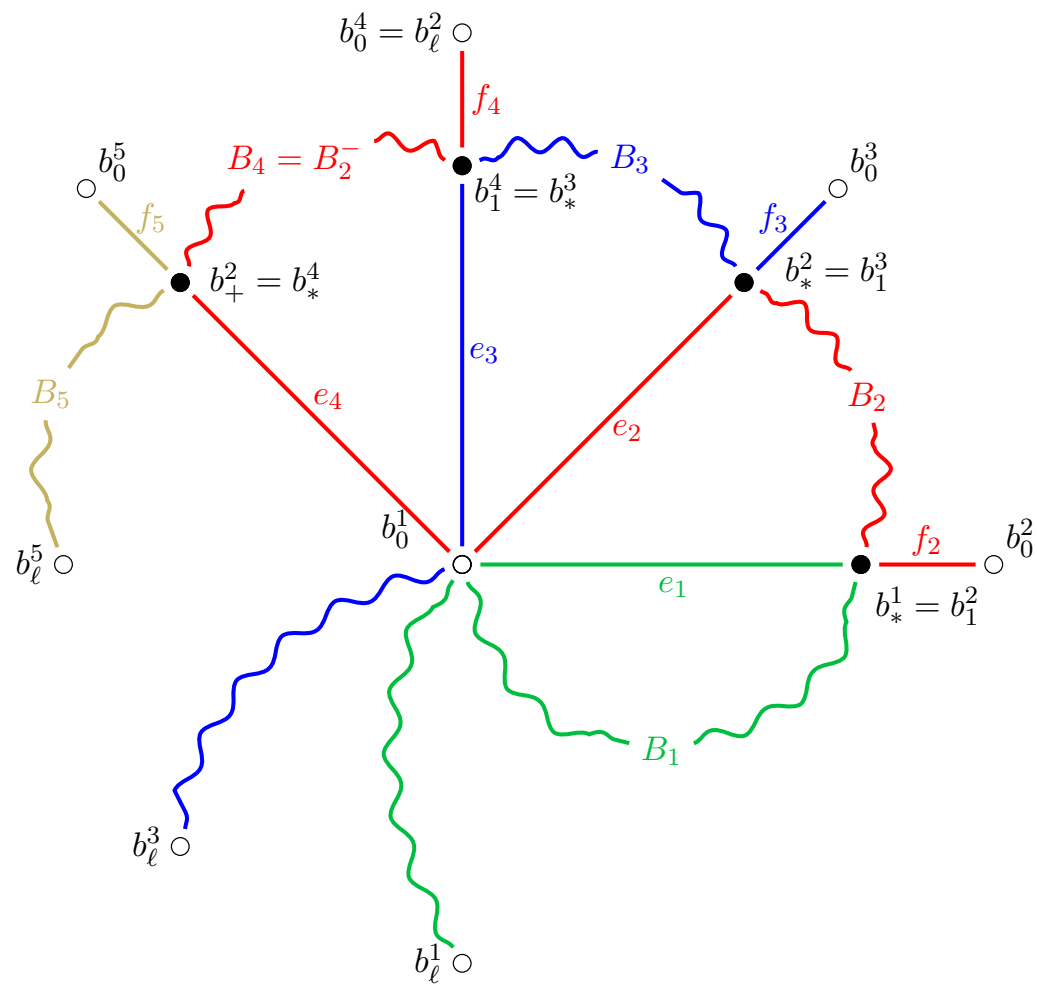

Figura 3.3: Uma sequência aumentante $S=B_{1} B_{2} B_{3} B_{4} B_{5}$, onde $B_{4}=B_{2}^{-}$.

Definição 3.2 (Sequência aumentante). Sejam $\ell$ e $r \geq 2$ inteiros positivos e seja $\mathcal{B}$ uma $\ell$-decomposição de um grafo $G$. Seja $S=B_{1} B_{2} \cdots B_{r}$ uma $\mathcal{B}$-sequência, onde $B_{i}=b_{0}^{i} b_{1}^{i} \cdots b_{\ell}^{i}$ para $i=1, \ldots, r$. Dizemos que $S$ é uma sequência aumentante de $\mathcal{B}$ se

(i) $\bar{B}_{1}$ não é um caminho, $d_{\bar{B}_{1}}\left(b_{0}^{1}\right)>1$, e $b_{0}^{2} \notin V\left(B_{1}\right)$;

(ii) se $i \in\{2, \ldots, r\}$, então $B_{i-1}$ contém $b_{0}^{1}$ e $b_{1}^{i}=b_{*}^{i-1}$;

e para $i \geq 3$, vale o seguinte:

(iii) se $\bar{B}_{i-1} \neq \bar{B}_{h}$ para todo $h<i-1$, então $b_{0}^{i} \notin V\left(\bar{B}_{i-1}-f_{i-1}+e_{i-2}\right)$;

(iv) se $\bar{B}_{i-1}=\bar{B}_{1}$, então $b_{0}^{i} \notin V\left(\bar{B}_{1}-e_{1}+f_{2}-f_{i-1}+e_{i-2}\right)$;

(v) se $\bar{B}_{i-1}=\bar{B}_{h}$ para algum $1<h<i-1$, então $b_{0}^{i} \notin V\left(\bar{B}_{h}-f_{h}+e_{h-1}-e_{h}+f_{h+1}-f_{i-1}+e_{i-2}\right)$.

Se, além disso, $b_{0}^{1} \notin V\left(B_{r}\right)$, então dizemos que $S$ é uma sequência aumentante perfeita.

Lembremos que, sempre que removemos arestas de uma trilha (como nos itens (iii)-(v)), nós também removemos vértices isolados que podem resultar após a remoção das arestas. Também observamos que (iv) implica que se $\bar{B}_{i-1}=\bar{B}_{1}$ então $B_{i} \neq B_{i-1}$ (isso será usado mais tarde). Em particular, (ii) implica que $\bar{B}_{i} \neq \bar{B}_{i-1}$ para todo $i=2, \ldots, r$. De fato, se $\bar{B}_{i}=\bar{B}_{i-1}$ para algum $i \in\{2, \ldots, r\}$, então o vértice $b_{1}^{i}$ tem grau 4 em $B_{i}$ (pois é incidente às arestas $b_{0}^{i} b_{1}^{i}, b_{1}^{i} b_{2}^{i}$, $b_{s(i-1)-2}^{i-1} b_{*}^{i-1}$, e $\left.b_{*}^{i-1} b_{s(i)}^{i-1}\right)$, porém não é vértice final de $B_{i}$ (veja Figura $3.2 \mathrm{~d}$ ), uma contradição com $\bar{B}_{i}$ ser uma trilha peculiar. Além disso, note que tanto $b_{0}^{1} b_{1}^{1} \cdots b_{s(1)}^{1}$ como $b_{*}^{i-1} b_{0}^{1}+b_{1}^{i} \cdots b_{s(i)}^{i}$ induzem circuitos em $G$, logo, como $G$ não tem arestas paralelas, temos $s(i) \geq 3$, para todo $i$. 
A Figura 3.3 ilustra uma sequência aumentante de comprimento 5 e, em particular, temos que a trilha $\bar{B}_{2}$ se repete em $S$ (isso é, $B_{4}=B_{2}^{-}$), de forma a ilustrar o item (v) da Definição 3.2. Note também que $B_{5}$ não contém o vértice $b_{0}^{1} \mathrm{e}$, portanto, a trilha $\bar{B}_{5}-f_{5}+e_{4}$ é um caminho.

A ideia principal por trás de nosso resultado central é que, se podemos encontrar uma sequência aumentante perfeita em uma dada decomposição em rastros, então podemos melhorar essa decomposição. Assim, as condições enunciadas na Definição 3.2 têm o propósito de permitir a troca de arestas dos elementos de uma sequência aumentante perfeita. Tal troca será realizada começando pelo primeiro elemento $B_{1}$ e então indo de $B_{i}$ para $B_{i+1}$. Se os elementos são todos distintos, então a simples troca que mencionamos na motivação é suficiente, contanto que os itens (i)-(iii) estejam satisfeitos. Porém, como as trilhas correspondentes a alguns rastros podem se repetir, precisamos das condições (iv) e (v). Note que a condição do item (iv) requer que se $\bar{B}_{i}=\bar{B}_{1}$, então o vértice inicial de $B_{i+1}$ não pode pertencer à trilha correspondente ao rastro que foi obtido de $B_{1}$ (isso é, a trilha $\left.\bar{B}_{1}-e_{1}+f_{2}-f_{i}+e_{i-1}\right)$. O item (v) requer que se $\bar{B}_{i}=\bar{B}_{h}$ para algum $1<h<i-1$, então o vértice inicial de $B_{i+1}$ não pertence à trilha correspondente ao rastro que foi obtido de $B_{h}$. Nesse caso, uma vez que $1<h<i$, o rastro original $B_{h}$ sofreu três trocas de arestas. A primeira é a troca da aresta $f_{h}$ pela aresta $e_{h-1}$; a segunda é a troca da aresta $e_{h}$ pela aresta $f_{h+1}$; a terceira é a troca da aresta $f_{i}$ pela aresta $e_{i-1}$. Logo, a condição no item (v) reflete essa troca tripla de arestas sofrida por $B_{h}$. Para entender essa ideia, observe as modificações da trilha $\bar{B}_{2}=\bar{B}_{4}$ da sequência aumentante mostrada na Figura 3.3 (veja o passo-a-passo mostrado na Figura 3.4 onde as trocas das arestas entre os rastros é ilustrada pela troca de cores das arestas).

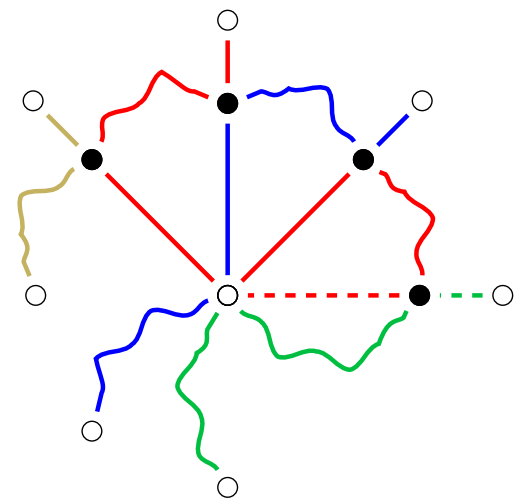

(a)

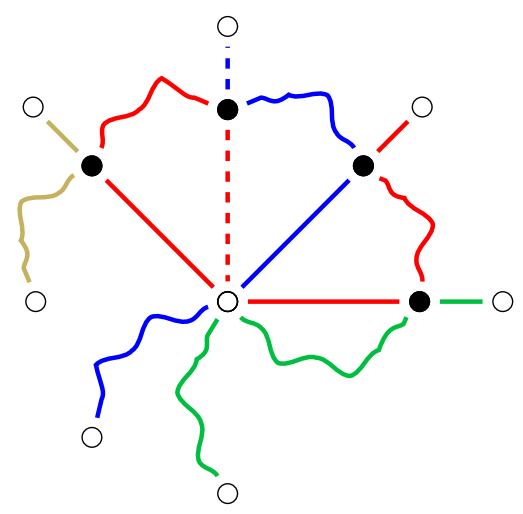

(c)

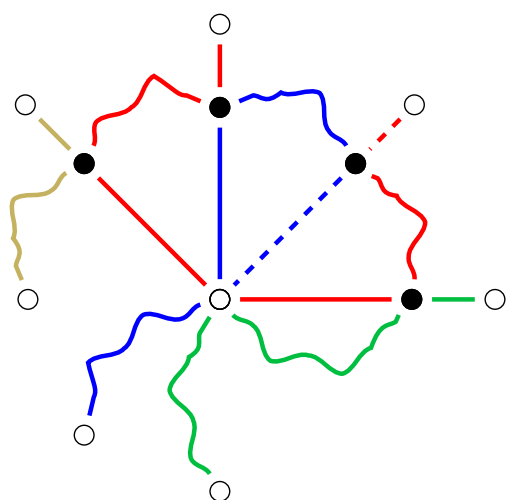

(b)

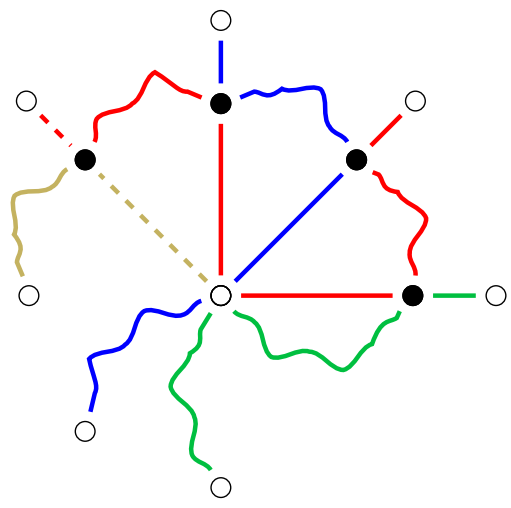

(d)

Figura 3.4: Ilustração de como lidar com a sequência aumentante perfeita da Figura 3.3. Em cada passo, as arestas tracejadas são as arestas que são trocadas. 
Como veremos, sequências aumentantes de $\ell$-decomposições $\mathcal{B}$ têm um número finito de elementos. Para provar isso (Corolário 3.4), mostramos primeiramente o seguinte resultado.

Lema 3.3. Sejam $\ell$ e $r \geq 2$ inteiros positivos e seja $\mathcal{B}$ uma $\ell$-decomposição de um grafo $G$. Se $S=$ $B_{1} B_{2} \cdots B_{r}$ é uma sequência aumentante de $\mathcal{B}$, então $b_{*}^{i} \neq b_{*}^{j}$ para todo $i, j$ com $1 \leq i<j \leq r-1$.

Demonstração. Sejam $\ell, r, \mathcal{B}$ e $S=B_{1} B_{2} \cdots B_{r}$ como na hipótese do lema, onde $B_{i}=b_{0}^{i} b_{1}^{i} \cdots b_{\ell}^{i}$ para $i=1, \ldots, r$. Queremos provar que $\left\{b_{*}^{1}, b_{*}^{2}, \ldots, b_{*}^{r-1}\right\}$ é um conjunto de elementos distintos.

Afirmação $A: b_{*}^{j} \neq b_{*}^{1}$, para $j=2, \ldots, r-1$.

Para $j=2$ o resultado é imediato. De fato, lembremos que $e_{i}=b_{*}^{i} b_{0}^{1} \in E\left(B_{i}\right)$. Se $b_{*}^{2}=b_{*}^{1}$, então $e_{1}=e_{2}$, isto é, $\bar{B}_{1}$ e $\bar{B}_{2}$ têm uma aresta em comum. Mas então, $\bar{B}_{1}=\bar{B}_{2}$, uma contradição (à Definição 3.2 (i)). Agora suponha que $j \geq 3$. Seja $j$ o menor índice para o qual $b_{*}^{j}=b_{*}^{1}$. Como no caso anterior, concluímos que $\bar{B}_{j}=\bar{B}_{1}$. Uma vez que $S$ é uma $\mathcal{B}$-sequência, temos que $B_{j}=B_{1}^{-}$ ou $B_{j}=B_{1}$. Se $B_{j}=B_{1}^{-}$, então $b_{*}^{j}=b_{+}^{1}$. Mas $b_{+}^{1} \neq b_{*}^{1}$. Logo, $b_{*}^{j} \neq b_{*}^{1}$, uma contradição. Se $B_{j}=B_{1}$, então $b_{0}^{j}=b_{0}^{1}$ e $f_{j}=f_{1}$. Então $b_{1}^{j}=b_{*}^{j-1}$ (Definição 3.2 (ii)). Assim, $f_{j}=b_{0}^{j} b_{1}^{j}=b_{0}^{1} b_{*}^{j-1}=e_{j-1}$, ou seja, $f_{j} \in B_{j-1}$. Uma vez que $f_{j}=f_{1} \in B_{1}$, concluímos que $\bar{B}_{j-1}=\bar{B}_{1}$. Logo, $\bar{B}_{j-1}=\bar{B}_{1}=\bar{B}_{j}$, isto é, $\bar{B}_{j-1}=\bar{B}_{j}$, uma contradição (veja a observação após a Definição 3.2).

Afirmação $B: b_{*}^{i} \neq b_{*}^{j}$ para todo $i, j$ com $2 \leq i<j \leq r-1$.

Suponha que isso não valha. Seja $i$ o menor inteiro tal que existe $j>i$ tal que $b_{*}^{i}=b_{*}^{j}$. Nesse caso, $e_{i}=b_{*}^{i} b_{0}^{1}=b_{*}^{j} b_{0}^{1}=e_{j}$, e logo $\bar{B}_{i}=\bar{B}_{j}$. Então, temos que $B_{j}=B_{i}$ ou $B_{j}=B_{i}^{-}$. Se $B_{j}=B_{i}^{-}$, então $b_{*}^{j}=b_{+}^{i} \neq b_{*}^{i}$, uma contradição. Se $B_{j}=B_{i}$, então $b_{0}^{j}=b_{0}^{i}$ e $b_{1}^{j}=b_{1}^{i}$. Porém, temos que $b_{1}^{j}=b_{*}^{j-1}$ e $b_{1}^{i}=b_{*}^{i-1}$, de onde concluímos que $b_{*}^{j-1}=b_{*}^{i-1}$, uma contradição à escolha de $i$.

Corolário 3.4. Sejam $\ell$ e $r \geq 2$ inteiros positivos e seja $\mathcal{B}$ uma $\ell$-decomposição de um grafo $G$. Se $S=B_{1} B_{2} \cdots B_{r}$ é uma sequência aumentante de $\mathcal{B}$, então cada $B_{i}$ occorre no máximo uma vez em $S$. Além disso, se $\bar{B}_{i}=\bar{B}_{j}$ para algum par $i, j$ com $1 \leq i<j \leq r-1$, então $B_{j}=B_{i}^{-}$.

Demonstração. Sejam $\ell, r, \mathcal{B}$ e $S=B_{1} B_{2} \cdots B_{r}$ como na hipótese do corolário. Seja $B_{i}=b_{0}^{i} b_{1}^{i} \cdots b_{\ell}^{i}$, para $i=1, \ldots, r$. Suponha, por contradição, que $B_{i}=B_{j}$ para algum par $i, j \operatorname{com} 1 \leq i<j \leq r-1$. Nesse caso, $e_{i}=e_{j}$, e então, $b_{*}^{i}=b_{*}^{j}$, uma contradição ao Lema 3.3. Logo, uma vez que $S$ é uma $\mathcal{B}$-sequência e $B_{i} \neq B_{j}$, se $\bar{B}_{i}=\bar{B}_{j}$, então $B_{j}=B_{i}^{-}$.

O Corolário 3.4 implica que qualquer sequência aumentante de uma $\ell$-decomposição é finita.

\subsection{Arestas pendentes e rastro-decomposições completas}

Todos os conceitos definidos nesta seção referem-se a $\ell$-decomposições $\mathcal{B}$ de um grafo $G$, para um inteiro positivo $\ell$. Lembramos que qualquer rastro em $\mathcal{B}$ tem exatamente dois vértices finais, mesmo se eles coincidirem. Para $B$ em $\mathcal{B}$, denotamos por $\tau(B)$ o número de vértices finais de $B$ que têm grau maior que $1 \mathrm{em} \bar{B}$. Temos que $\tau(B) \in\{0,1,2\}$, e $\tau(B)=0$ se e somente se $\bar{B}$ é um caminho. A Figura 3.2a ilustra os casos em que $\tau(B)=1$, enquanto as Figuras 3.2b-f ilustram os casos em que $\tau(B)=2$. Assim, defina $\tau(\mathcal{B})=\sum_{B \in \mathcal{B}} \tau(B)$.

Seja $u v$ uma aresta de $G$, e seja $B$ o elemento de $\mathcal{B}$ que contém $u v$. Se $B=x_{0} x_{1} \cdots x_{\ell} \operatorname{com} x_{0}=u$ e $x_{1}=v$, ou $x_{\ell}=u$ e $x_{\ell-1}=v$, então dizemos que $u v$ é uma aresta quase-pendente em $v$ na decomposição $\mathcal{B}$. Se, além disso, $d_{\bar{B}}(u)=1$, então dizemos que uv é uma aresta pendente em $v$ 
na decomposição $\mathcal{B}$. Denotamos por $q \operatorname{Pend}(v, \mathcal{B})$ (resp. $\operatorname{Pend}(v, \mathcal{B}))$ o número de arestas quasependentes (resp. pendentes) em $v$ na decomposição $\mathcal{B}$. Note que toda aresta pendente em um vértice $v$ é também uma aresta quase-pendente em $v$. Logo, temos que qPend $(v, \mathcal{B}) \geq \operatorname{Pend}(v, \mathcal{B})$ para todo vértice $v$. Seja $k$ um inteiro positivo. Dizemos que $\mathcal{B}$ é $k$-pré-completa se qPend $(v, \mathcal{B})>k$ para todo $v$ em $V(G)$. Se $\operatorname{Pend}(v, \mathcal{B})>k$ para todo $v$ em $V(G)$, então dizemos que $\mathcal{B}$ é $k$-completa. Dizemos também que $\mathcal{B}$ é completa, se $\mathcal{B}$ é $k$-completa para algum $k$.

Seja $J$ o conjunto das arestas de $G$ que são iniciais ou finais de rastros de $\mathcal{B}$. Considere uma orientação $O$ de $J$ com a seguinte propriedade: se $u v$ é uma aresta de $J$ e $B$ é o elemento de $\mathcal{B}$ que contém $u v$, onde $B=x_{0} x_{1} \cdots x_{\ell} \operatorname{com} x_{0}=u$ e $x_{1}=v$, ou $x_{\ell}=u$ e $x_{\ell-1}=v$, então $u v$ é orientada $($ em $O)$ de $v$ para $u$. Note que $d_{O}^{+}(v)=q \operatorname{Pend}(v, \mathcal{B})$ para todo vértice $v$ de $G$. Além disso, para cada $v$ de $G$ definimos $\mathcal{B}(v):=d_{O}^{-}(v)$. Notamos que se $\mathcal{B}$ é uma $\ell$-decomposição de $G$, então $\sum_{v \in V(G)} \mathcal{B}(v)=2|\mathcal{B}|=2|E(G)| / \ell$, porque cada elemento de $\mathcal{B}$ tem exatamente dois vértices finais (contados com multiplicidade). O próximo lema é a ferramenta principal na prova do Lema de Desemaranhamento (Lema 3.9).

Lema 3.5. Sejam $k$ e $\ell$ inteiros positivos e seja $\mathcal{B}$ uma $\ell$-decomposição $k$-completa de um grafo $G$. $S e \mathcal{B}$ contém uma sequência aumentante perfeita, então existe uma $\ell$-decomposição $\mathcal{B}^{\prime}$ de $G$ com as seguintes propriedades.

- $\tau\left(\mathcal{B}^{\prime}\right)<\tau(\mathcal{B})$;

- $\mathcal{B}^{\prime}(v)=\mathcal{B}(v)$ para todo $v \in V(G)$;

- $\mathcal{B}^{\prime}$ é k-completa.

Demonstração. Sejam $k, \ell$ e $\mathcal{B}$ como na hipótese do lema. Suponha que $S=B_{1} \cdots B_{r}$ seja uma sequência aumentante perfeita de $\mathcal{B}$, onde $S=B_{1} \cdots B_{r}$, e $B_{i}=b_{0}^{i} b_{1}^{i} \cdots b_{\ell}^{i}$ para $i=1, \ldots, r$.

A prova segue por indução no número de elementos de $S$, denotado por $|S|$. Note que pela definição de sequência aumentante perfeita, temos que $b_{0}^{1} \notin V\left(B_{r}\right)$. Portanto, $|S|=r \geq 2$.

Pelo item (ii) da Definição $3.2, b_{1}^{2}=b_{*}^{1}$. Sejam $\bar{B}_{1}^{\prime}=\bar{B}_{1}-e_{1}+f_{2}$ e $\bar{B}_{2}^{\prime}=\bar{B}_{2}-f_{2}+e_{1}$. Ou seja, $\bar{B}_{1}^{\prime}$ e $\bar{B}_{2}^{\prime}$ são obtidos de $\bar{B}_{1}$ e $\bar{B}_{2}$ pela troca das arestas $e_{1}$ e $f_{2}$. Considere os seguintes rastros correspondentes a essas trilhas: $B_{1}^{\prime}=b_{0}^{2} X^{-} b_{+}^{1} \cdots b_{\ell}^{1}$, onde $X=b_{0}^{1} b_{1}^{1} \cdots b_{*}^{1}$; e $B_{2}^{\prime}=b_{0}^{1} b_{1}^{2} b_{2}^{2} \cdots b_{\ell}^{2}$. É fácil ver que $B_{1}^{\prime}$ e $B_{2}^{\prime}$ são $\ell$-rastros de $G$, e além disso, $\bar{B}_{1} \cup \bar{B}_{2}=\bar{B}_{1}^{\prime} \cup \bar{B}_{2}^{\prime}$.

Seja $\mathcal{B}^{\prime}=\mathcal{B}-B_{1}-B_{2}+B_{1}^{\prime}+B_{2}^{\prime}$. Claramente, $\mathcal{B}^{\prime}$ é uma $\ell$-decomposição de $G$. Pelo item (i) da Definição $3.2, d_{\bar{B}_{1}}\left(b_{0}^{1}\right)>1$ e $b_{0}^{2} \notin V\left(B_{1}\right)$, de onde concluímos que $d_{\bar{B}_{1}^{\prime}}\left(b_{0}^{2}\right)=1$ e $\tau\left(B_{1}^{\prime}\right) \leq \tau\left(B_{1}\right)-1$. A seguir, provamos que $\mathcal{B}^{\prime}(v)=\mathcal{B}(v)$ para todo $v \in V(G)$, e que $\mathcal{B}^{\prime}$ é $k$-completa.

Afirmação 3.6. $\mathcal{B}^{\prime}(v)=\mathcal{B}(v)$ para todo $v \in V(G)$.

Demonstração. Sejam $J$ e $J^{\prime}$ os conjuntos das arestas de $G$ que são iniciais ou finais de rastros de $\mathcal{B}$ e de $\mathcal{B}^{\prime}$, respectivamente. Temos que $J^{\prime}=J-b_{0}^{1} b_{1}^{1}+e_{1}$. Sejam $O$ e $O^{\prime}$ orientações de $J$ e $J^{\prime}$, respectivamente, como acima, i.e, tal que $d_{O}^{-}(v)=\mathcal{B}(v)$ e $d_{O^{\prime}}^{-}(v)=\mathcal{B}^{\prime}(v)$ para todo vértice $v$ de $G$. Note que, como $\mathcal{B} \cap \mathcal{B}^{\prime}=\mathcal{B}-B_{1}-B_{2}=\mathcal{B}^{\prime}-B_{1}^{\prime}-B_{2}^{\prime}$, temos que as arestas em $J \cap J^{\prime}$ são orientadas da mesma forma em $O$ e em $O^{\prime}$. Logo, $d_{O^{\prime}}^{-}(v)=d_{O}^{-}(v)$ para todo vértice $v$ em $V(G)-b_{0}^{1}$. Para concluir, note que tanto $b_{0}^{1} b_{1}^{1}$ quanto $e_{1}$ são orientadas entrando em $b_{0}^{1}$. Logo, $d_{O}^{-}\left(b_{0}^{1}\right)=d_{O^{\prime}}^{-}\left(b_{0}^{1}\right)$ e $\mathcal{B}^{\prime}(v)=d_{O^{\prime}}^{-}(v)=d_{O}^{-}(v)=\mathcal{B}(v)$ para todo $v \in V(G)$. 
Afirmação 3.7. $\mathcal{B}^{\prime}$ é $k$-completa.

Demonstração. Vamos provar que $\operatorname{Pend}\left(v, \mathcal{B}^{\prime}\right)>k$ para todo $v \in V(G)$. Note que $\operatorname{Pend}(v, \mathcal{B})=$ $\operatorname{Pend}\left(v, \mathcal{B}-B_{1}-B_{2}\right)+\operatorname{Pend}\left(v,\left\{B_{1}, B_{2}\right\}\right)$ e $\operatorname{Pend}\left(v, \mathcal{B}^{\prime}\right)=\operatorname{Pend}\left(v, \mathcal{B}^{\prime}-B_{1}^{\prime}-B_{2}^{\prime}\right)+\operatorname{Pend}\left(v,\left\{B_{1}^{\prime}, B_{2}^{\prime}\right\}\right)$. Como $\mathcal{B}^{\prime}-B_{1}^{\prime}-B_{2}^{\prime}=\mathcal{B}-B_{1}-B_{2}$, temos que $\operatorname{Pend}\left(v, \mathcal{B}-B_{1}-B_{2}\right)=\operatorname{Pend}\left(v, \mathcal{B}^{\prime}-B_{1}^{\prime}-B_{2}^{\prime}\right)$. Assim, precisamos apenas mostrar que $\operatorname{Pend}\left(v,\left\{B_{1}^{\prime}, B_{2}^{\prime}\right\}\right) \geq \operatorname{Pend}\left(v,\left\{B_{1}, B_{2}\right\}\right)$. Para isso, basta notar que as possíveis arestas pendentes em $\left\{B_{1}, B_{2}\right\}$ estão contidas em $\left\{b_{\ell-1}^{1} b_{\ell}^{1}, b_{1}^{2} b_{0}^{2}, b_{\ell-1}^{2} b_{\ell}^{2}\right\}$ (a aresta $b_{1}^{1} b_{0}^{1}$ é quase-pendente, mas não é pendente em $\left.\left\{B_{1}, B_{2}\right\}\right)$, e que as arestas pendentes em $\left\{B_{1}^{\prime}, B_{2}^{\prime}\right\}$ estão contidas em $\left\{b_{\ell-1}^{1} b_{\ell}^{1}, b_{1}^{2} b_{0}^{2}, b_{\ell-1}^{2} b_{\ell}^{2}\right\}+b_{*}^{1} b_{0}^{1}$. Então, $\operatorname{Pend}\left(v, \mathcal{B}^{\prime}\right) \geq \operatorname{Pend}(v, \mathcal{B})>k$. Portanto, $\mathcal{B}^{\prime}$ é $k$-completa.

Suponha que $|S|=2$. Como $S$ é uma sequência aumentante perfeita, temos que $b_{0}^{1} \notin V\left(B_{2}\right)$ e, assim, $d_{\bar{B}_{2}^{\prime}}\left(b_{0}^{1}\right)=1$. Logo, $\tau\left(B_{2}^{\prime}\right) \leq \tau\left(B_{2}\right)$ e, portanto, a seguinte desigualdade vale.

$$
\tau\left(\mathcal{B}^{\prime}\right)=\tau(\mathcal{B})-\tau\left(B_{1}\right)-\tau\left(B_{2}\right)+\tau\left(B_{1}^{\prime}\right)+\tau\left(B_{2}^{\prime}\right)<\tau(\mathcal{B})
$$

Logo, $\mathcal{B}^{\prime}$ é a $\ell$-decomposição procurada.

Assim, podemos supor que $|S|=r>2$ e que o lema vale quando $\mathcal{B}$ contém uma sequência aumentante perfeita $S^{\prime} \operatorname{com} r-1$ elementos. Uma vez que $|S|>2$, pelo item (ii) da Definição 3.2 temos que $b_{1}^{2}=b_{*}^{1}$ e $b_{s(2)}^{2}=b_{0}^{1}$, onde $s(2) \geq 3$. No caso $|S|=2$, tínhamos $b_{0}^{1} \notin V\left(B_{2}\right)$, mas agora temos que $b_{0}^{1} \in V\left(B_{2}\right)$; logo, neste caso podemos concluir somente que $\tau\left(\mathcal{B}^{\prime}\right) \leq \tau(\mathcal{B})$. Veja Figura 3.4a. No que segue, mostramos que a decomposição $\mathcal{B}^{\prime}$ obtida acima contém uma sequência aumentante perfeita $S^{\prime}$ que pode ser obtida de $S$.

Uma vez que $S$ é uma sequência aumentante de $\mathcal{B}$, pelo Corolário 3.4, todo $\bar{B}_{i}$ aparece no máximo duas vezes em $S$ e se $\bar{B}_{i}=\bar{B}_{j}$ para $1 \leq i<j \leq r$, então, $B_{j}=B_{i}^{-}$. Seja $S^{\prime}=C_{2} C_{3} \cdots C_{r}$, onde $C_{2}=B_{2}^{\prime}$ e para $3 \leq i \leq r$,

$$
C_{i}= \begin{cases}B_{1}^{--} & \text {se } B_{i}=B_{1}^{-} \\ B_{2}^{\prime-} & \text { se } B_{i}=B_{2}^{-} \\ B_{i} & \text { caso contrário. }\end{cases}
$$

Observe que $S^{\prime}$ é precisamente a $\mathcal{B}^{\prime}$-sequência que é obtida de $S$ ao retirarmos $B_{1}$, e substituirmos a possível ocorrência de $B_{1}^{-}$por $B_{1}^{\prime-}$, a ocorrência de $B_{2}$ por $B_{2}^{\prime}$, e a possível ocorrência de $B_{2}^{-}$ por $B_{2}^{\prime-}$. Se $\bar{B}_{i}=\bar{B}_{1}$ para algum $i>1$, temos que $C_{i}=B_{1}^{\prime-}$. Além disso, note que as diferenças entre os rastros $C_{i}$ e $B_{1}^{-}$acontecem todas após o índice $s(i)$, i.e, $c_{j}^{i}=b_{j}^{i}$ para todo $j \leq s(i)$; a única diferença entre os rastros $C_{2}$ e $B_{2}$ é o primeiro vértice e, portanto, $c_{j}^{2}=b_{j}^{2}$ para todo $j \geq 1, \operatorname{logo}$, se $C_{i}=B_{2}^{\prime-}$ para algum $i>2$, então $c_{j}^{i}=b_{j}^{i}$ para todo $j \leq \ell-1$. Concluímos que

$$
c_{j}^{i}=b_{j}^{i} \text { para todo } 1 \leq j \leq s(i) \text { e } i \geq 2 \text {, e que } c_{0}^{i}=b_{0}^{i} \text { para todo } i \geq 3
$$

Como $b_{s(i)}^{i}=b_{0}^{1}=c_{0}^{2}$, temos que $s(i)$ é o menor índice tal que $c_{s(i)}^{i}=c_{0}^{2}=b_{0}^{1}$, para $2 \leq i \leq r$. Assim, faz sentido usarmos o mesmo índice $s(i)$ para $S^{\prime}$. Consequentemente, temos que $c_{*}^{i}=b_{*}^{i}$ para todo $2 \leq i \leq r-1$. Note que com isso temos $c_{*}^{i} c_{s(i)}^{i}=b_{*}^{i} b_{s(i)}^{i}=e_{i}$ para todo $i \geq 2$, e $c_{0}^{i} c_{1}^{i}=b_{0}^{i} b_{1}^{i}=f_{i}$ para todo $i \geq 3$. Afirmamos que $S^{\prime}$ é uma sequência aumentante perfeita. Para isso, iremos verificar 
a validade de cada um dos itens da Definição 3.2.

Item (i): $\bar{C}_{2}$ não é um caminho, $d_{\bar{C}_{2}}\left(c_{0}^{2}\right)>1$, e $c_{0}^{3} \notin V\left(C_{2}\right)$.

Uma vez que $C_{2}=B_{2}^{\prime}$, temos que $c_{0}^{2}=b_{0}^{1}$. Além disso, uma vez que $\bar{B}_{2}^{\prime}=\bar{B}_{2}-f_{2}+e_{1}$, as arestas $e_{1}$ e $e_{2}$ estão em $\bar{B}_{2}^{\prime}=C_{2}$ e são incidentes a $c_{0}^{2}$. Logo, $d_{\bar{C}_{2}}\left(c_{0}^{2}\right)>1$. Agora, vamos provar que $c_{0}^{3} \notin V\left(C_{2}\right)=V\left(B_{2}^{\prime}\right)$. Uma vez que $B_{2} \neq B_{1}$, pelo item (iii) da Definição 3.2 (aplicada a $S$ com $i=3)$ temos que $b_{0}^{3} \notin V\left(\bar{B}_{2}-f_{2}+e_{1}\right)$. Mas, pela definição de $C_{2}$ temos que $\bar{C}_{2}=\bar{B}_{2}-f_{2}+e_{1}$. Como, devido a (3.1), temos que $c_{0}^{3}=b_{0}^{3}$, então $c_{0}^{3} \notin V\left(C_{2}\right)$.

Item (ii): se $i \in\{3, \ldots, r\}$, o elemento $C_{i-1}$ contém $c_{0}^{2}$, e $c_{1}^{i}=c_{*}^{i-1}$.

Fixe $i \in\{3, \ldots, r\}$. Uma vez que $b_{0}^{1} \in B_{i-1}$, pela definição de $C_{i}$ temos que $c_{0}^{2}=b_{0}^{1} \in C_{i-1}$. Além disso, como observado anteriormente, temos que $c_{j}^{i}=b_{j}^{i}$ para todo $1 \leq j \leq s(i)$ e $i \geq 2$. Logo, temos que $c_{1}^{i}=b_{1}^{i}$ para todo $2 \leq i \leq r$ e $c_{*}^{i-1}=b_{*}^{i-1}$ para todo $3 \leq i \leq r$. Pelo item (ii) da definição 3.2 aplicado a $S$, temos que $b_{1}^{i}=b_{*}^{i-1}$. Logo se $i \geq 3$, temos que $c_{1}^{i}=b_{1}^{i}=b_{*}^{i-1}=c_{*}^{i-1}$.

Item (iii): para $i \geq 4$, se $\bar{C}_{i-1} \neq \bar{C}_{h}$ para todo $h<i-1$, então $c_{0}^{i} \notin V\left(\bar{C}_{i-1}-f_{i-1}+e_{i-2}\right)$.

Fixe $i \in\{3, \ldots, r\}$ e suponha que $\bar{C}_{i-1} \neq \bar{C}_{h}$ para todo $2 \leq h<i-1$. Note que $\bar{C}_{i-1} \neq \bar{B}_{2}^{\prime}$. Primeiramente consideramos o caso em que $\bar{C}_{i-1}=\bar{B}_{1}^{\prime}$ ou, equivalentemente, $C_{i-1}=B_{1}^{\prime-}$. Logo, $B_{i-1}=B_{1}^{-}$, e pelo item (iv) da Definição 3.2 aplicado a $S$, temos que $b_{0}^{i} \notin V\left(\bar{B}_{1}-e_{1}+f_{2}-f_{i-1}+\right.$ $e_{i-2}$ ). Como $C_{i-1}=B_{1}^{\prime-}$, temos que $\bar{C}_{i-1}=\bar{B}_{1}-e_{1}+f_{2}$. Uma vez que, devido a (3.1), temos que $c_{0}^{i}=b_{0}^{i}$ para todo $i \geq 3$, então $c_{0}^{i} \notin V\left(\bar{B}_{1}-e_{1}+f_{2}-f_{i-1}+e_{i-2}\right)=V\left(\bar{C}_{i-1}-f_{i-1}+e_{i-2}\right)$. Agora suponha que $\bar{C}_{i-1} \neq \bar{B}_{1}^{\prime}$. Então, $\bar{B}_{i-1} \neq \bar{B}_{1}$. Mas sabemos que $\bar{C}_{i-1} \neq \bar{C}_{h}$ para todo $h<i-1$, o que implica que $B_{i-1} \neq B_{h}$ para todo $h<i-1$. Do item (iii) da Definição 3.2 (aplicado a $S$ ), temos que $b_{0}^{i} \notin V\left(\bar{B}_{i-1}-f_{i-1}+e_{i-2}\right)$. Uma vez que $c_{0}^{i}=b_{0}^{i}$, temos que $c_{0}^{i} \notin V\left(\bar{B}_{i-1}-f_{i-1}+e_{i-2}\right)$. Uma vez que $\bar{C}_{i-1} \neq \bar{B}_{1}^{\prime}, \bar{B}_{2}^{\prime}$, concluímos que $C_{i-1}=B_{i-1}$ e, portanto, $c_{0}^{i} \notin V\left(\bar{C}_{i-1}-f_{i-1}+e_{i-2}\right)$.

Item (iv): para $i \geq 4$, se $\bar{C}_{i-1}=\bar{C}_{2}$, então $c_{0}^{i} \notin V\left(\bar{C}_{2}-e_{2}+f_{3}-f_{i-1}+e_{i-2}\right)$.

Fixe $i \in\{4, \ldots, r\}$ e suponha que $\bar{C}_{i-1}=\bar{C}_{2}$. Nesse caso, temos que $C_{i-1}=B_{2}^{\prime-}$. Pelo item (v) da Definição 3.2 aplicado a $S$ com parâmetros $i$ e $h=2$, temos que

$$
b_{0}^{i} \notin V\left(\bar{B}_{2}-f_{2}+e_{1}-e_{2}+f_{3}-f_{i-1}+e_{i-2}\right) .
$$

Como $B_{2}^{\prime}=B_{2}-f_{2}+e_{1}$, temos que $V\left(\bar{B}_{2}-f_{2}+e_{1}-e_{2}+f_{3}-f_{i-1}+e_{i-2}\right)=V\left(\bar{B}_{2}^{\prime}-e_{2}+f_{3}-f_{i-1}+e_{i-2}\right)$. Assim, como $C_{i-1}=B_{2}^{\prime-}$ e $c_{0}^{i}=b_{0}^{i}$, temos que

$$
\begin{aligned}
c_{0}^{i}=b_{0}^{i} & \notin V\left(\bar{B}_{2}^{\prime}-e_{2}+f_{3}-f_{i-1}+e_{i-2}\right) \\
& =V\left(\bar{C}_{2}-e_{2}+f_{3}-f_{i-1}+e_{i-2}\right) .
\end{aligned}
$$

Item (v): para $i \geq 4$, se $\bar{C}_{i-1}=\bar{C}_{h}$ para algum $2<h<i-1$, então $c_{0}^{i} \notin V\left(\bar{C}_{h}-f_{h}+e_{h-1}-\right.$ $\left.e_{h}+f_{h+1}-f_{i-1}+e_{i-2}\right)$. 
Fixe $i \in\{4, \ldots, r\}$ e suponha que $\bar{C}_{i-1}=\bar{C}_{h}$ para algum $2<h<i-1$. Nesse caso, temos que $C_{i-1}=C_{h}^{-}$, e então $\bar{B}_{i-1}=\bar{B}_{h}$ e, pelo Corolário $3.4, \bar{C}_{i-1} \neq \bar{B}_{1}^{\prime}, \bar{B}_{2}^{\prime}$. Portanto, temos que $C_{h}=B_{h}$ e $C_{i-1}=B_{i-1}$. Pelo item (v) da Definição 3.2 aplicado a $S$, concluímos que

$$
{ }^{\prime} b_{0}^{i} \notin V\left(\bar{B}_{h}-f_{h}+e_{h-1}-e_{h}+f_{h+1}-f_{i-1}+e_{i-2}\right) .
$$

Assim, como $c_{0}^{i}=b_{0}^{i}$, temos que

$$
\begin{aligned}
c_{0}^{i}=b_{0}^{i} & \notin V\left(\bar{B}_{h}-f_{h}+e_{h-1}-e_{h}+f_{h+1}-f_{i-1}+e_{i-2}\right) \\
& =V\left(\bar{C}_{h}-f_{h}+e_{h-1}-e_{h}+f_{h+1}-f_{i-1}+e_{i-2}\right) .
\end{aligned}
$$

Concluímos a prova de que $S^{\prime}$ é uma sequência aumentante de $\mathcal{B}^{\prime}$. Mas, como $S$ é uma sequência aumentante perfeita de $\mathcal{B}$, sabemos que $b_{0}^{1} \notin V\left(B_{r}\right)$ (então, claramente $\bar{B}_{r} \neq \bar{B}_{1}, \bar{B}_{2}$ ). Mas, como $c_{0}^{2}=b_{0}^{1}$, concluímos que $S^{\prime}$ é uma sequência aumentante perfeita de $\mathcal{B}^{\prime}$. Uma vez que $\left|S^{\prime}\right|=r-1$, pela hipótese de indução, $G$ admite uma $\ell$-decomposição $k$-completa $\mathcal{B}^{\prime \prime}$ tal que $\tau\left(\mathcal{B}^{\prime \prime}\right)<\tau\left(\mathcal{B}^{\prime}\right) \leq \tau(\mathcal{B})$ e $\mathcal{B}^{\prime \prime}(v)=\mathcal{B}^{\prime}(v)=\mathcal{B}(v)$ para todo vértice $v$ de $G$.

O próximo lema mostra como construir $\mathcal{B}$-sequências aumentantes perfeitas. Lembremos que, para um rastro $B$, denotamos por $\tau(B)$ o número de vértices finais de $B$ que têm grau maior que 1 , e para uma rastro-decomposição $\mathcal{B}$, denotamos por $\tau(\mathcal{B})$ o somatório $\sum_{B \in \mathcal{B}} \tau(B)$.

Lema 3.8. Sejam $\ell$ um inteiro positivo, $G$ um grafo, e $\mathcal{B}$ uma $\ell$-decomposição de $G$. Se $\mathcal{B}$ é viável e $\tau(\mathcal{B})>0$, então $\mathcal{B}$ contém uma sequência aumentante perfeita.

Demonstração. Sejam $\ell, G$ e $\mathcal{B}$ como na hipótese do lema. Primeiramente, vamos mostrar que $\mathcal{B}$ contém uma sequência aumentante. Como $\tau(\mathcal{B})>0$, a rastro-decomposição $\mathcal{B}$ contém um rastro $B_{1}$ que não induz um caminho. Seja $B_{1}=b_{0}^{1} b_{1}^{1} \cdots b_{\ell}^{1}$, onde $d_{\bar{B}_{1}}\left(b_{0}^{1}\right)>1$. Como $\mathcal{B}$ é viável, existe uma aresta $b_{*}^{1} w$ pendente em $b_{*}^{1}$ (note que $b_{*}^{1}$ está contido no circuito $b_{0}^{1} b_{1}^{1} \cdots b_{s(1)}^{1}$ de $B_{1}$ ) em $\mathcal{B}$ tal que $w \notin V\left(B_{1}\right)$. Seja $B_{2}$ o elemento de $\mathcal{B}$ que contém a aresta $b_{*}^{1} w$. Então, é fácil verificar que $B_{1} B_{2}$ é uma sequência aumentante de $\mathcal{B}$.

Seja $S=B_{1} B_{2} \cdots B_{r}$ uma sequência aumentante maximal de $\mathcal{B}$. Suponha, por contradição, que $S$ não seja perfeita, i.e, $b_{0}^{1} \in V\left(B_{r}\right)$.

Agora vamos mostrar como obter um elemento $B_{r+1}$ de $\mathcal{B}$ tal que $S^{\prime}=B_{1} \cdots B_{r} B_{r+1}$ é uma sequência aumentante, contradizendo a maximalidade de $S$. Primeiramente, note que, para $S$, a validade do item (i) da Definição 3.2 não depende de $B_{i}$ para todo $i \geq 3$; e a validade dos itens (ii)-(v) da Definição 3.2 para um índice fixo $i \in\{2, \ldots, r\}$ não depende de $B_{j}$ para todo $j>i$. Além disso, seja $T=a_{0} a_{1} \cdots a_{\ell}$ um elemento de $\mathcal{B}$ que contém uma aresta pendente em $b_{*}^{r}$. Então $a_{1}=b_{*}^{r}$ ou $a_{\ell-1}=b_{*}^{r}$. Assim, podemos escolher $B_{r+1}=T$ ou $B_{r+1}=T^{-}$de tal forma que $b_{1}^{r+1}=b_{*}^{r}$. Logo, o item (ii) da Definição 3.2 vale para $i=r+1$. Antes de continuar, para cada $i$ considere a $\ell$-trilha $Q_{i}=\bar{B}_{i}-f_{i}+e_{i-1}$. Note que $Q_{i}$ contém o circuito $b_{0}^{1} b_{1}^{i} b_{2}^{i} \cdots b_{*}^{i} b_{0}^{1}$, que claramente contém $b_{*}^{i}$. Agora, note que exatamente um dos itens a seguir vale: (a) $\bar{B}_{r} \neq \bar{B}_{h}$, para todo $h<r$; (b) $\bar{B}_{r}=\bar{B}_{1}$; ou (c) $\bar{B}_{r}=\bar{B}_{h}$ para algum $1<h<r$. Claramente, (a), (b), (c) são as condições dos itens (iii), (iv), (v) da Definição 3.2 (para $i=r+1$ ), respectivamente. Mostramos que, em cada caso, a conclusão também vale. 
(a) Nesse caso, pela viabilidade de $\mathcal{B}$, considerando $T=\bar{B}_{r}-f_{r}+e_{r-1}$ e $v=b_{*}^{r}$, existe uma aresta $b_{*}^{r} z$, pendente em $b_{*}^{r}$, tal que $z \notin V\left(\bar{B}_{r}-f_{r}+e_{r-1}\right)$. Seja $B_{r+1}$ o elemento de $\mathcal{B}$ contendo $b_{*}^{r} z$. Como observado acima, podemos supor sem perda de generalidade que $z=$ $b_{0}^{r+1}$ e $b_{1}^{r+1}=b_{*}^{r}$ (caso contrário, $z=b_{\ell}^{r+1}$ e escolhemos $B_{r+1}^{-}$no lugar de $B_{r+1}$ ). Logo, $b_{0}^{r+1}=z \notin V\left(\bar{B}_{r}-f_{r}+e_{r-1}\right)$. Portanto, o item (iii) da Definição 3.2 vale para $i=r+1$.

(b) Nesse caso, temos que $B_{r}=B_{1}^{-}=b_{\ell}^{1} P_{r} b_{*}^{r} b_{0}^{1} b_{*}^{1} P_{1}^{-} b_{0}^{1}$, onde $P_{r}=b_{2}^{r} b_{r}^{3} \cdots b_{s(r)-2}^{r}$ e $P_{1}=$ $b_{1}^{1} b_{2}^{1} \cdots b_{s(1)-2}^{1}$. Pela viabilidade de $\mathcal{B}$, considerando $v=b_{*}^{r}$ e $T=\bar{B}_{1}-e_{1}+f_{2}-f_{r}+e_{r-1}$ (note que $T$ é induzido pelo rastro $b_{0}^{1} P_{r} b_{*}^{r} b_{0}^{1} P_{1}^{-} b_{*}^{1} b_{0}^{2}$ e contém o circuito $b_{0}^{1} b_{\ell-1}^{1} b_{\ell-2}^{1} \cdots b_{*}^{r} b_{0}^{1}$ ), existe uma aresta $b_{*}^{r} z$ pendente em $b_{*}^{r}$ tal que $z \notin V\left(\bar{B}_{1}-e_{1}+f_{2}-f_{r}+e_{r-1}\right)$. Seja $B_{r+1}$ o elemento de $\mathcal{B}$ contendo $b_{*}^{r} z$. Como no caso anterior, podemos supor que $z=b_{0}^{r+1} \notin V\left(\bar{B}_{1}-e_{1}+f_{2}-f_{r}+e_{r-1}\right)$ e $b_{1}^{r+1}=b_{*}^{r}$. Portanto, o item (iv) da Definição 3.2 vale para $i=r+1$.

(c) Nesse caso, temos que $B_{r}=b_{\ell}^{h} P_{r} b_{*}^{r} b_{0}^{1} b_{*}^{h} P_{h}^{-} b_{0}^{h}$, onde $P_{r}=b_{1}^{r} b_{2}^{r} \cdots b_{s(r)-2}^{r}$ e $P_{h}=b_{1}^{h} b_{2}^{h} \cdots b_{s(h)-2}^{h}$. Como $S$ é uma sequência aumentante, pelo item (ii) da Definição $3.2, b_{1}^{h}=b_{*}^{h-1}$ e $b_{1}^{h+1}=b_{*}^{h}$. Logo, como $b_{s(h-1)}^{h-1}=b_{s(h)}^{h}=b_{0}^{1}$, concluímos que $e_{h-1}=b_{1}^{h} b_{0}^{1}$ e $f_{h+1}=b_{*}^{h} b_{0}^{h+1}$ são arestas de $B_{h-1}$ e $B_{h+1}$, respectivamente. Tome $T=\bar{B}_{r}-b_{1}^{h} b_{0}^{h}+b_{1}^{h} b_{0}^{1}-b_{*}^{h} b_{0}^{1}+b_{*}^{h} b_{0}^{h+1}-f_{r}+e_{r-1}=$ $\bar{B}_{r}-f_{h}+e_{h-1}-e_{h}+f_{h+1}-f_{r}+e_{r-1}$ (note que $T$ é induzido pelo rastro $b_{0}^{1} P_{r} b_{*}^{r} b_{0}^{1} b_{*}^{h} P_{h} b_{0}^{h+1}$ ). Como $T$ contém o circuito $b_{0}^{1} b_{1}^{r} b_{2}^{r} \cdots b_{*}^{r} b_{0}^{1}$, pela viabilidade de $\mathcal{B}$, considerando $v=b_{*}^{r}$ e $T$, existe uma aresta $b_{*}^{r} z$ pendente em $b_{*}^{r}$ tal que $z \notin V\left(\bar{B}_{r}-f_{h}+e_{h-1}-e_{h}+f_{h+1}-f_{r}+e_{r-1}\right)$. Seja $B_{r+1}$ o elemento de $\mathcal{B}$ contendo $b_{*}^{r} z$. Como no caso anterior, podemos supor que $z=b_{0}^{r+1} \notin V(T)$ e $b_{1}^{r+1}=b_{*}^{r}$. Portanto, o item (v) da Definição 3.2 vale para $i=r+1$.

Provamos que existe um elemento $B_{r+1}$ de $\mathcal{B}$ tal que $S^{\prime}=B_{1} B_{2} \cdots B_{r} B_{r+1}$ é uma sequência aumentante de $\mathcal{B}$, uma contradição à maximalidade de $S$. Portanto, $S$ é uma sequência aumentante perfeita.

O próximo lema, o resultado principal dessa seção, segue diretamente dos lemas 3.5 e 3.8.

Lema 3.9 (Lema de Desemaranhamento). Sejam $\ell$ um inteiro positivo e $G$ um grafo. Se $\mathcal{B}$ é uma $\ell$-decomposição viável e $k$-completa de $G$ e $\tau(\mathcal{B})>0$, então existe uma $\ell$-decomposição $\mathcal{B}^{\prime}$ de $G$ com as seguintes propriedades.

- $\tau\left(\mathcal{B}^{\prime}\right)<\tau(\mathcal{B})$;

- $\mathcal{B}^{\prime}(v)=\mathcal{B}(v)$ para todo $v \in V(G)$;

- $\mathcal{B}^{\prime}$ é k-completa.

Demonstração. Sejam $\ell, G$ e $\mathcal{B}$ como na hipótese do lema. Como $\mathcal{B}$ é viável e $\tau(\mathcal{B})>0$, pelo Lemma $3.8, \mathcal{B}$ contém uma sequência aumentante perfeita. Como $\mathcal{B}$ é $k$-completa e contém uma sequência aumentante perfeita, pelo Lemma 3.5 , existe uma $\ell$-decomposição $\mathcal{B}^{\prime}$ de $G$ com as propriedades desejadas. 


\section{Capítulo 4}

\section{Decomposição de grafos regulares em caminhos de comprimento fixo}

Neste capítulo aplicamos o Lema de Desemaranhamento desenvolvido no Capítulo 3 para obter decomposições em caminhos de comprimento fixo de uma grande família de grafos regulares.

Kotzig [Kot57] e, independentemente, Bouchet e Fouquet [BF83] provaram que um grafo 3-regular admite uma $P_{3}$-decomposição se e somente se ele contém um emparelhamento perfeito. A prova desse resultado introduz a estrutura de prova que utilizamos nesse capítulo, e que generalizamos para grafos altamente aresta-conexos no Capítulo 5. Seja $M$ um emparelhamento perfeito em um grafo 3-regular $G$. Temos que $G-M$ é um 2-fator de $G$. Escolha uma orientação Euleriana para $G-M$ e, para cada aresta $x y$ em $M$, seja $P_{x y}$ o subgrafo de $G$ que contém $x y$ e as arestas em $G-M$ que saem de $x$ e $y$ na orientação escolhida. O conjunto $\left\{P_{x y}: x y \in M\right\}$ é uma $P_{3}$-decomposição de $G$ (veja Figura 4.1).
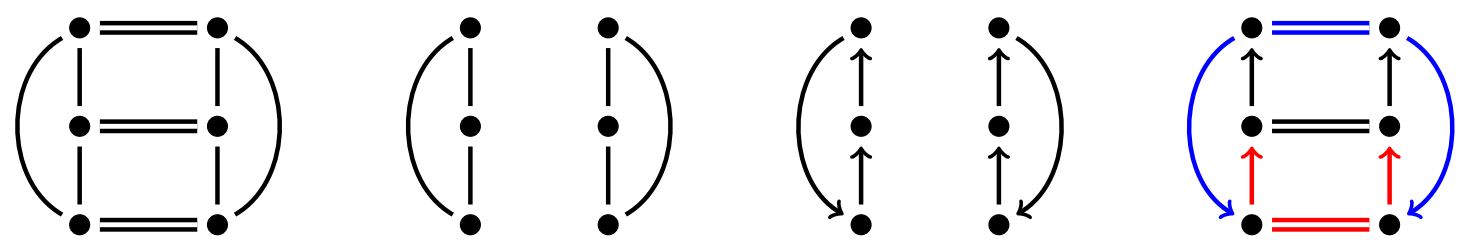

Figura 4.1: Ilustração da prova do Teorema de Kotzig, e Bouchet e Fouquet.

Kotzig perguntou quais são as condições necessárias e suficientes para que um grafo $\ell$-regular ímpar $G$ seja decomponível em caminhos de comprimento $\ell$. Uma condição necessária é a existência de um $\lfloor\ell / 2\rfloor$-fator (e, consequentemente, um $\lceil\ell / 2\rceil$-fator) em $G$. Para ver isso, tome uma decomposição $\mathcal{D}$ de $G$ em $\ell$-caminhos e, para cada caminho $P=x_{0} x_{1} \cdots x_{\ell} \in \mathcal{D}$, considere o subconjunto $F_{P}=\left\{x_{1} x_{2}, x_{3} x_{4}, \ldots, x_{\ell-2} x_{\ell-1}\right\}$; o subgrafo de $G$ dado por $F=\cup_{P \in \mathcal{D}} F_{P}$ é um $\lfloor\ell / 2\rfloor$ fator de $G$, e o subgrafo $G-E(F)$ é um $\lceil\ell / 2\rceil$-fator de $G$ (veja Figura 4.2). Favaron, Genest, e Kouider [FGK10] mostraram que a existência desse $[\ell / 2\rfloor$-fator não é suficiente para a existência de uma $P_{\ell}$-decomposição (veja Figura 4.3).

Por outro lado, eles mostraram que, para um grafo 5 -regular admitir uma $P_{5}$-decomposição, é suficiente conter um emparelhamento perfeito e não ter circuitos de comprimento quatro, e propuseram a seguinte conjectura. 


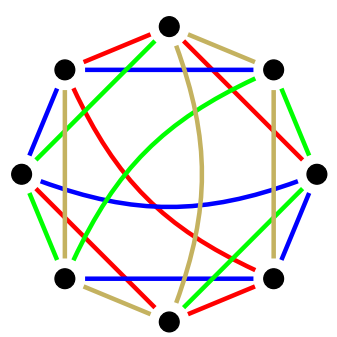

(a)

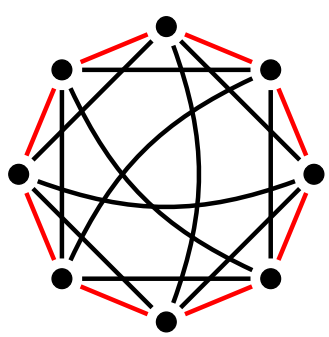

(b)

Figura 4.2: Grafo 5-regular decomposto (a) em cópias de $P_{5}$ e (b) em um 2-fator e um 3-fator.

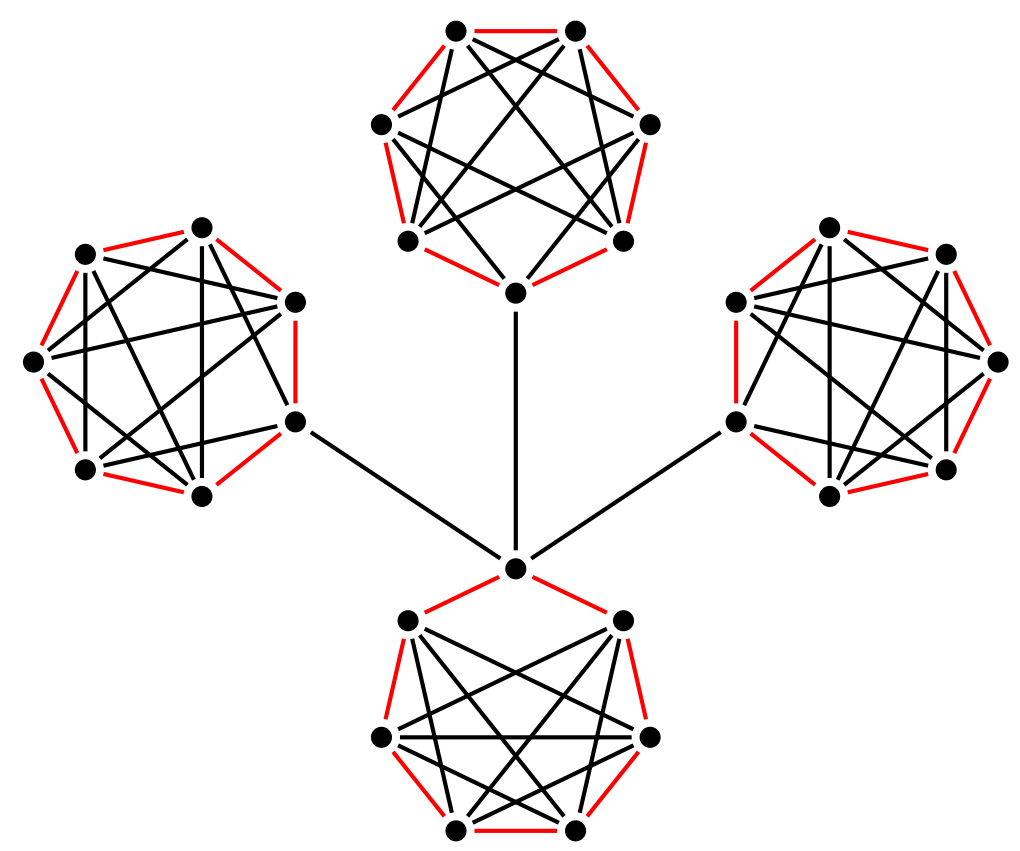

Figura 4.3: Grafo 5-regular que contém 2-fator, mas não admite $P_{5}$-decomposição.

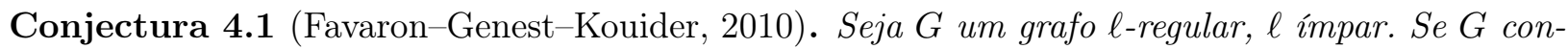
tém um emparelhamento perfeito, então $G$ admite uma $P_{\ell}$-decomposição.

Recentemente, provamos [BMW15] que todo grafo 5-regular livre de triângulos e contendo um emparelhamento perfeito admite uma $P_{5}$-decomposição, confirmando a validade da Conjectura 4.1 para grafos 5-regulares com cintura pelo menos 4. Na Seção 4.2 apresentamos uma generalização do resultado em [BMW15]. Mais precisamente, provamos (veja Teorema 4.13) que grafos $\ell$-regulares ímpares com cintura pelo menos $\ell-1$ e que contêm emparelhamento perfeito admitem

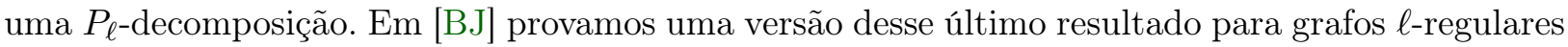
pares. Provamos que grafos $\ell$-regulares Eulerianos com cintura pelo menos $\ell-2$, e contendo dois emparelhamentos perfeitos disjuntos admitem uma $\left\{P_{\ell-1}, P_{\ell}, P_{\ell+1}\right\}$-decomposição. Em particular, o resultado em [BJ] confirma, para essa família de grafos, a validade de uma conjectura de Gallai que afirma que qualquer grafo conexo simples com $n$ vértices pode ser decomposto em no máximo $\lceil n / 2\rceil$ caminhos (veja [Lov68, Bon14]).

Seja $\mathcal{D}$ uma decomposição de um grafo $G$ em trilhas. Dado um vértice $v$ de $G$, denotamos por $\mathcal{D}(v)$ o número de elementos de $\mathcal{D}$ que contêm $v$ como vértice final. Dizemos que $\mathcal{D}$ é balanceada se $\mathcal{D}(u)=\mathcal{D}(v)$ para todo $u, v \in V(G)$. Note que $\mathcal{D}(v) \equiv d(v)(\bmod 2)$ para todo $v$ em $V(G)$. O seguinte fato será usado frequentemente, 
Fato 4.2. Sejam $\ell, m$ e $n$ inteiros positivos.

(i) Se lé ímpar e $\mathcal{D}$ é uma decomposição de um grafo $\ell$-regular $G$ em trilhas de comprimento $\ell$, então $\mathcal{D}$ é balanceada.

(ii) Se nm é par e $\mathcal{D}$ é uma decomposição em trilhas de comprimento $\ell$ balancedada de um grafo $m \ell$-regular $G$ com $n$ vértices, então $\mathcal{D}(v)=m$ para todo vértice $v$ de $G$.

Demonstração. Provamos os dois itens separadamente. Primeiramente, note que dada uma decomposição em trilhas $\mathcal{D}$ de um grafo $G$, temos que $\sum_{v \in V(G)} \mathcal{D}(v)=2|\mathcal{D}|$, uma vez que cada trilha tem exatamente dois vértices finais.

(i) Sejam $\ell, \mathcal{D}$ e $G$ como na hipótese. Como $d(v)=\ell$ é ímpar para todo vértice $v$ de $G$, temos que $\mathcal{D}(v) \geq 1$ para todo vértice $v$ de $G$. Assim, se $\mathcal{D}(u) \geq 3$ para algum $u$ em $V(G)$, temos que

$$
2|\mathcal{D}|=\sum_{v \in V(G)} \mathcal{D}(v) \geq n+1=\frac{2 n \ell}{2 \ell}+1=2 \frac{|E(G)|}{\ell}+1>2|\mathcal{D}| .
$$

Portanto, $\mathcal{D}(v)=1$ para todo vértice $v$ de $G$ e $\mathcal{D}$ é balanceada.

(ii) Sejam $\ell G$ e $\mathcal{D}$ como no enunciado, e seja $n=|V(G)|$. Primeiramente, temos que $|E(G)|=$ $n m \ell / 2$. Como cada elemento em $\mathcal{D}$ tem exatamente $\ell$ arestas, temos que $|\mathcal{D}|=n m / 2$. Como $\mathcal{D}$ é balanceada, temos que $\mathcal{D}(u)=\mathcal{D}(v)$ para todos $u, v \in V(G)$. Assim, dado vértice $u$ em $V(G)$, temos que

$$
n \mathcal{D}(u)=\sum_{v \in V(G)} \mathcal{D}(v)=2|\mathcal{D}|=n m .
$$

Portanto, $\mathcal{D}(u)=m$.

Heinrich, Liu e Yu [HLY99] provaram que se $G$ é um grafo $3 m$-regular que contém um $m$ fator, então $G$ admite uma $P_{3}$-decomposição balanceada (nesse caso, $\mathcal{D}(v)=m$ para todo $v$ em $V(G))$. Kouider e Lonc [KL99] provaram que se $G$ é um grafo $2 \ell$-regular com cintura $g \geq(\ell+3) / 2$, então $G$ admite uma $P_{\ell}$-decomposição balanceada. Eles também provaram que se $\ell$ é par, então todo grafo bipartido $\ell$-regular com cintura $g \geq(\ell+3) / 2$ admite uma $P_{\ell}$-decomposição. Além disso, eles propuseram a seguinte conjectura.

Conjectura 4.3 (Kouider-Lonc, 1999). Todo grafo $2 \ell$-regular admite uma $P_{\ell}$-decomposição balanceada.

Assim, para resolver a Conjectura 4.3, falta prová-la para grafos com cintura $g<(\ell+3) / 2$. Kouider e Lonc [KL99] provaram-na para $\ell=3$. A Conjectura 4.3 é um fortalecimento (para caminhos) da Conjectura 1.1 sobre decomposições de grafos $2 \ell$-regulares em árvores com $\ell$ arestas. Recentemente, provamos [BT] que a Conjectura 4.3 vale quando $\ell=4$, i.e, se $G$ é um grafo 8-regular, então $G$ admite $P_{4}$-decomposição balanceada.

Neste capítulo, consideramos o problema de obter $P_{\ell}$-decomposições balanceadas de grafos $k \ell$-regulares, para inteiros positivos $k$. Propusemos a seguinte conjectura, que consiste de uma generalização da Conjectura 4.1 (item (i)) e de uma forma equivalente da Conjectura 4.3 (item (ii)). A equivalência entre a Conjectura 4.3 e o item (ii) da Conjectura 4.4 segue do Teorema de Fatorização de Petersen (veja o Teorema 2.1). 
Conjectura 4.4 (Botler-Mota-Oshiro-Wakabayashi, 2015). Para $m$ e $\ell$ inteiros positivos, valem as seguintes afirmações.

(i) Se $\ell$ é impar, então todo grafo $m \ell$-regular que contém um $m$-fator admite uma $P_{\ell}$-decomposição balanceada.

(ii) Todo grafo $2 m \ell$-regular admite uma $P_{\ell}$-decomposição balanceada.

Note que o item (ii) da Conjectura 4.4 é falso se no lugar de $2 m \ell$ considerarmos $m \ell$ com $m$ ímpar e $\ell$ par. De fato, se $G$ é um grafo $m \ell$-regular com $n$ vértices, então $|E(G)|=n m \ell / 2$. Se $G$

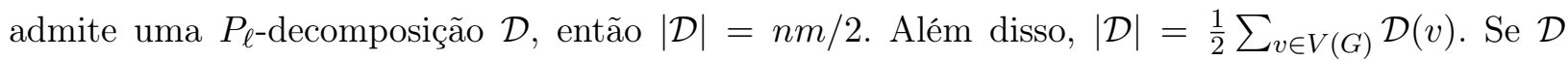
é balanceada, então $|\mathcal{D}|=n \mathcal{D}(v) / 2$ para todo $v \in V(G)$. Portanto, $n \mathcal{D}(v) / 2=n m / 2$, e logo $\mathcal{D}(v)=m$ para todo $v \in V(G)$. Mas, uma vez que $\mathcal{D}$ é uma decomposição em caminhos e $G$ é Euleriano, $\mathcal{D}(v)$ tem que ser par para todo $v \in V(G)$, uma contradição.

Provamos neste capítulo que a Conjectura 4.4 (i) vale para grafos com cintura pelo menos $g$ tal que $m>2\lfloor(\ell-2) /(g-2)\rfloor$, e a Conjectura 4.4 (ii) vale para grafos com cintura pelo menos $g$ tal que $m>\lfloor(\ell-2) /(g-2)\rfloor$. Em particular, provamos a Conjectura 4.4 (i) para $m, \ell$ tais que $m \geq 2 \ell-1$, e a Conjectura 4.4 (ii) para $m, \ell$ tais que $m \geq \ell-1$. Nossos resultados principais, Teoremas 4.10 e 4.11, são os seguintes: sejam $g, \ell$ e $m$ inteiros positivos com $g \geq 3$. (i) Se $\ell$ é ímpar e $m>2\lfloor(\ell-2) /(g-2)\rfloor$, então todo grafo $m \ell$-regular com cintura pelo menos $g$ que contém um $m$-fator admite uma $P_{\ell}$-decomposição balanceada. (ii) Se $m>\lfloor(\ell-2) /(g-2)\rfloor$, então todo grafo $2 m \ell$-regular com cintura pelo menos $g$ admite uma $P_{\ell}$-decomposição balanceada.

Note que, para $\ell$ ímpar, nosso resultado implica que, para todo inteiro $m \geq 3$, se $G$ é um grafo $m \ell$-regular com cintura pelo menos $\ell-1$ e contém um $m$-fator, então $G$ admite uma $P_{\ell^{-}}$ decomposição balanceada. Os resultados em [BMW15] podem ser facilmente generalizados para obter esse resultado para $m=1$, e um teorema em [KL99] trata do caso $m=2$. Nós discutimos esse resultado na Seção 4.2.1. Se $G$ é um grafo bipartido, então provamos que o item (i) da Conjectura 4.4 vale para todo $m>\ell$, enquanto o item (ii) vale para todo $m>\ell / 2$ (veja a Subseção 4.2.2).

\subsection{Decomposição de grafos regulares com cintura prescrita}

Os resultados principais dessa seção, Teoremas 4.10 e 4.11, dão condições suficientes para grafos regulares com cintura prescrita admitirem decomposições em caminhos de comprimento dado. Para isso, primeiramente aplicamos o Teorema de Fatorização de Petersen (Teorema 2.1) para obter uma decomposição inicial em trilhas, e então aprimoramos a estrutura dessa decomposição (veja Lema 4.5) para aplicar o Lema de Desemaranhamento (Lema 3.9), e obter a decomposição em caminhos desejada.

Lema 4.5. Sejam $g, k, \ell$ e $r$ inteiros positivos com $g \geq 3$ e seja $G$ um grafo com cintura pelo menos g. Se $r \geq\lfloor(\ell-2) /(g-2)\rfloor$ e $G$ admite uma $\ell$-decomposição $(k+r)$-pré-completa $\mathcal{B}$, então $G$ admite uma $\ell$-decomposição $k$-completa $\mathcal{B}^{\prime}$ tal que $\mathcal{B}^{\prime}(v)=\mathcal{B}(v)$ para todo vértice $v$ de $G$.

Demonstração. Sejam $g, k, \ell, r$ e $G$ como na hipótese. Seja $\mathcal{B}$ uma $\ell$-decomposição $(k+r)$-précompleta de $G$, e seja $\mathcal{B}^{\prime}$ uma $\ell$-decomposição $(k+r)$-pré-completa de $G$ tal que $\mathcal{B}^{\prime}(v)=\mathcal{B}(v)$ para todo vértice $v$ de $G$, e que maximiza $\sum_{v \in V(G)} \operatorname{Pend}\left(v, \mathcal{B}^{\prime}\right)$. Afirmamos que $\mathcal{B}^{\prime}$ é $k$-completa, i.e, $\operatorname{Pend}\left(v, \mathcal{B}^{\prime}\right)>k$ para cada vértice $v$ de $G$. 
Suponha, por contradição, que $\mathcal{B}^{\prime}$ não seja $k$-completa. Então, existe um vértice $u$ de $G$ tal que Pend $\left(u, \mathcal{B}^{\prime}\right) \leq k$. Uma vez que $\mathcal{B}^{\prime}$ é $(k+r)$-pré-completa, qPend $\left(u, \mathcal{B}^{\prime}\right) \geq k+r+1$. Logo, existem pelo menos $r+1$ arestas quase-pendentes em $u$ que não são arestas pendentes em $u$, digamos $u x_{1}, \ldots, u x_{r+1}$. Seja $T_{1}=y_{0} y_{1} \cdots y_{\ell}$ o elemento de $\mathcal{B}^{\prime}$ que contém $u x_{1}$, onde, sem perda de generalidade, $y_{0}=x_{1}$ e $y_{1}=u$, e seja $X=\left\{x_{1}, \ldots, x_{r+1}\right\}$. Sejam $x_{1}^{\prime}, \ldots, x_{s}^{\prime}$ os vértices de $X$ contidos em $V\left(T_{1}\right)$, ordenados por distância a $y_{1}$ na sequência $y_{1} y_{2} \cdots y_{\ell}$. Seja $\ell_{0}$ a distância de $y_{1}$ a $x_{1}^{\prime}$ em $T_{1}-y_{0} y_{1}$, e seja $\ell_{i}$ a distância de $x_{i}^{\prime}$ a $x_{i+1}^{\prime}$ em $T_{1}-y_{0} y_{1}$, para $0<i \leq s-1$ (veja Figura 4.4). Como a cintura de $G$ é pelo menos $g$, temos que $\ell_{0} \geq g-1$, e $\ell_{i} \geq g-2$ para $1 \leq i \leq s-1$. Como $x_{1}^{\prime}, \ldots, x_{s}^{\prime}$ estão ordenados por distância a $y_{1}$ em $T_{1}-y_{0} y_{1}$, temos que $\ell-1 \geq \sum_{i=0}^{s-1} \ell_{i} \geq g-1+(s-1)(g-2)=s(g-2)+1$. Portanto, $s \leq(\ell-2) /(g-2)$, o que implica que $s \leq r$. Como $|X|=r+1>s$, existe pelo menos um vértice em $X$, digamos $x_{p}$, que não é vértice de $T_{1}$.

Seja $T_{p}$ o elemento de $\mathcal{B}^{\prime}$ que contém $u x_{p}$. Podemos supor sem perda de generalidade que $T_{p}=z_{0} z_{1} \cdots z_{\ell}$, onde $z_{0}=x_{p}$ e $z_{1}=u=y_{1}$. Seja $T_{1}^{\prime}=x_{p} y_{1} \cdots y_{\ell}$ e $T_{p}^{\prime}=x_{1} z_{1} \cdots z_{\ell}$. Note que $\bar{T}_{1}^{\prime}=\bar{T}_{1}-u x_{1}+u x_{p}$ e $\bar{T}_{p}^{\prime}=\bar{T}_{p}-u x_{p}+u x_{1}$, e considere $\mathcal{B}^{\prime \prime}=\mathcal{B}^{\prime}-T_{1}-T_{p}+T_{1}^{\prime}+T_{p}^{\prime}$. Não é difícil verificar que $\mathcal{B}^{\prime \prime}(v)=\mathcal{B}^{\prime}(v)=\mathcal{B}(v)$ para todo $v$ em $V(G)$. Como $x_{p} \notin V\left(T_{1}\right)$, temos que $d_{\bar{T}_{1}^{\prime}}\left(x_{p}\right)=1$, o que implica que $u x_{p}$ é uma aresta pendente em $u$ na decomposição $\mathcal{B}^{\prime \prime}$. Portanto, $\sum_{v \in V(G)} \operatorname{Pend}\left(v, \mathcal{B}^{\prime \prime}\right)>$ $\sum_{v \in V(G)} \operatorname{Pend}\left(v, \mathcal{B}^{\prime}\right)$, uma contradição à maximalidade de $\sum_{v \in V(G)} \operatorname{Pend}\left(v, \mathcal{B}^{\prime}\right)$.

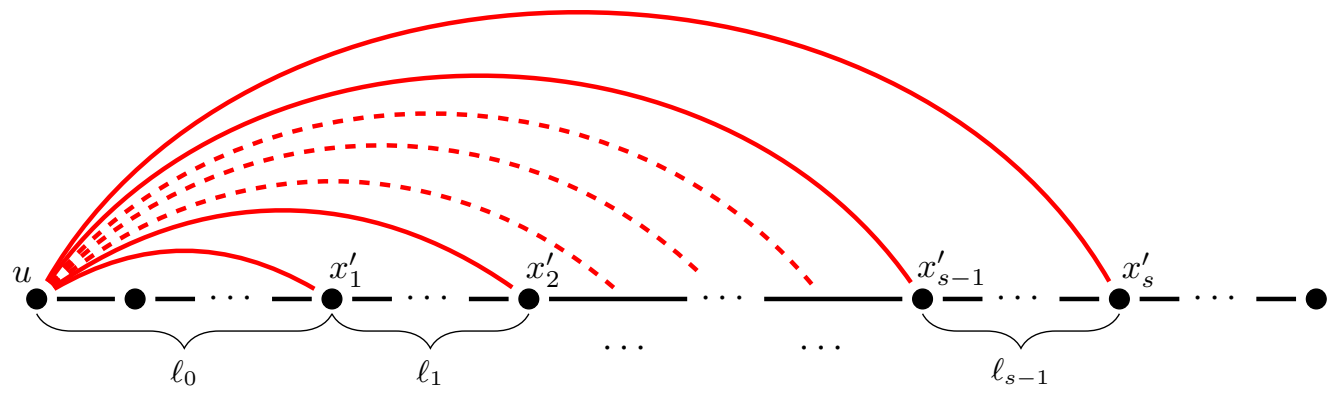

Figura 4.4: Obtenção de uma $\ell$-decomposição $k$-completa de uma $\ell$-decomposição $(k+r)$-pré-completa.

O lema a seguir fornece uma condição suficiente para uma $\ell$-decomposição completa ser viável.

Lema 4.6. Sejam $g, k$ e $\ell$ inteiros positivos com $g \geq 3$ e seja $G$ um grafo com cintura pelo menos $g$. Se $k \geq\lfloor(\ell-2) /(g-2)\rfloor$ e $\mathcal{B}$ é uma $\ell$-decomposição $k$-completa de $G$, então $\mathcal{B}$ é viável.

Demonstração. Sejam $g, k, \ell, G$ e $\mathcal{B}$ como na hipótese do lema. Fixe $v \in V(G)$, e seja $T$ uma $\ell$-trilha de $G$ que contém um circuito, digamos $C$, tal que $v \in V(C)$. Uma vez que $\mathcal{B}$ é $k$-completa, $\operatorname{Pend}(v, \mathcal{B})>k \geq\lfloor(\ell-2) /(g-2)\rfloor$. Sejam $v w_{1}, \ldots, v w_{\lfloor(\ell-2) /(g-2)\rfloor+1}$ arestas pendentes em $v$ na decomposição $\mathcal{B}$, e tome $W=\left\{w_{1}, \ldots, w_{\lfloor(\ell-2) /(g-2)\rfloor+1}\right\}$. Afirmamos que existe $w_{i} \in W$ tal que $w_{i} \notin V(T)$. Separe $T$ em $v$, obtendo duas trilhas $T_{1}$ e $T_{2}$ de comprimentos $c_{1}$ e $c_{2}$, respectivamente. Denote por $s_{i}$ o número de vértices de $W$ em $T_{i}$. Se $W \subseteq V(T)$, então temos que $|W| \leq s_{1}+s_{2}$. Suponha que $s_{1} \geq 1$. Sejam $w_{1}^{\prime}, \ldots, w_{s_{1}}^{\prime}$ os vértices de $W$ em $V\left(T_{1}\right)$, ordenados por distância a $v$ em $T_{1}$. Seja $\ell_{0}$ a distância em $T_{1}$ de $v$ a $w_{1}^{\prime}$, e $\ell_{i}$ a distância em $T_{1}$ de $w_{i}^{\prime}$ a $w_{i+1}^{\prime}$, para $i>0$. Como $G$ tem cintura pelo menos $g$, temos que $d_{0} \geq g-1$, e $\ell_{i} \geq g-2$, para $1 \leq i \leq s_{1}-1$. Portanto, $c_{1} \geq \sum_{i=0}^{s_{1}-1} \ell_{i} \geq g-1+\left(s_{1}-1\right)(g-2)=s_{1}(g-2)+1$. Analogamente, obtemos que se $s_{2} \geq 1$, então 
$c_{2} \geq s_{2}(g-2)+1$. Logo, se $s_{1}, s_{2} \geq 1$, então $s_{1}+s_{2} \leq(\ell-2) /(g-2)$, uma contradição. Agora, suponha que $s_{1}=0$. Como $v$ é um vértice interno de $T$, temos que $\ell-1 \geq c_{2} \geq s_{2}(g-2)+1$, o que implica que $s_{1}+s_{2}=s_{2} \leq(\ell-2) /(g-2)$. Em ambos os casos temos que $s_{1}+s_{2} \leq\lfloor(\ell-2) /(g-2)\rfloor$. Como $|W| \geq\lfloor(\ell-2) /(g-2)\rfloor+1$, temos que $W \nsubseteq V(T)$ e, portanto, existe um vértice $w_{i} \in W$ que não é um vértice de $T$.

Agora, usamos o lema acima para provar o resultado principal desta seção, que nos permite obter uma $\ell$-decomposição em caminhos a partir de uma $\ell$-decomposição $\lfloor(\ell-2) /(g-2)\rfloor$-completa de grafos com cintura pelo menos $g$.

Lema 4.7. Sejam $g, k$ e $\ell$ inteiros positivos com $g \geq 3$ e seja $G$ um grafo com cintura pelo menos $g$. Se $k \geq\lfloor(\ell-2) /(g-2)\rfloor$ e $\mathcal{B}$ é uma $\ell$-decomposição $k$-completa de $G$, então $G$ admite uma $\ell$-decomposição $k$-completa em caminhos $\mathcal{B}^{\prime}$ tal que $\mathcal{B}(v)=\mathcal{B}^{\prime}(v)$ para todo vértice $v$ de $G$.

Demonstração. Sejam $g, k, \ell, G$ e $\mathcal{B}$ como na hipótese do lema. Seja $\mathbb{B}$ o conjunto de todas as $\ell$-decomposições $k$-completas $\mathcal{B}^{\prime}$ de $G$ tal que $\mathcal{B}^{\prime}(v)=\mathcal{B}(v)$ para todo vértice $v$ de $G$. Pela hipótese, temos que $\mathbb{B} \neq \emptyset$. Seja $\tau^{*}=\min \left\{\tau\left(\mathcal{B}^{\prime}\right): \mathcal{B}^{\prime} \in \mathbb{B}\right\}$ e seja $\mathcal{B}_{\text {min }}$ um elemento de $\mathbb{B}$ tal que $\tau\left(\mathcal{B}_{\text {min }}\right)=\tau^{*}$. Se $\tau^{*}=0$, então $\mathcal{B}_{\min }$ é uma $\ell$-decomposição em caminhos e a prova está terminada. Então, suponha que $\tau^{*}>0$. Pelo Lema 4.6, $\mathcal{B}_{\min }$ é uma $\ell$-decomposição viável. Como $\tau\left(\mathcal{B}_{\min }\right)>0$, pelo Lema 3.9 aplicado com $k, \ell, G$, e $\mathcal{B}_{\text {min }}$, existe uma $\ell$-decomposição $k$-completa $\mathcal{B}^{\prime}$ de $G$ tal que $\tau\left(\mathcal{B}^{\prime}\right)<\tau\left(\mathcal{B}_{\text {min }}\right)=\tau^{*}$ e $\mathcal{B}^{\prime}(v)=\mathcal{B}(v)$ para todo vértice $v$ de $G$. Portanto, $\mathcal{B}^{\prime}$ é um elemento de $\mathbb{B}$ $\operatorname{com} \tau\left(\mathcal{B}^{\prime}\right)<\tau\left(\mathcal{B}_{\min }\right)$, uma contradição à minimalidade de $\tau^{*}$.

\subsubsection{Decomposições de grafos regulares}

As provas dos nossos resultados principais sobre $\ell$-decomposição seguem por indução em $\ell$. Para obter essas provas, primeiramente precisamos estender o conceito de balanceamento para decomposições em rastros. Seja então $\mathcal{B}$ uma rastro-decomposição de um grafo $G$. Lembremos que definimos $\mathcal{B}(v)$, para $v \in V(G)$, como o número de arestas de $G$ incidentes a $v$ que são arestas iniciais de rastros em $\mathcal{B}$ que começam em $v$, ou arestas finais de rastros em $\mathcal{B}$ que terminam em $v$. Analogamente à definição de decomposição balanceada em trilhas, dizemos que $\mathcal{B}$ é balanceada se $\mathcal{B}(u)=\mathcal{B}(v)$ para todo $u, v \in V(G)$. Note que $\mathcal{B}$ é balanceada se e somente se a decomposição em trilhas subjacente a $\mathcal{B}$ também é balanceada.

Os dois resultados seguintes constituem casos especiais dos teoremas que provaremos a seguir.

Teorema 4.8 (Heinrich-Liu-Yu [HLY99]). Seja $m$ um inteiro positivo. Se G é um grafo 3m-regular que contém um m-fator, então $G$ admite uma 3-decomposição em caminhos balanceada.

Proposição 4.9. Seja $m$ um inteiro positivo. Se $G$ é um grafo $4 m$-regular, então $G$ admite uma 2-decomposição em caminhos balanceada.

Demonstração. Considere uma orientação Euleriana de $G$. Como $G$ é $4 m$-regular, temos que $d^{+}(v)=$ $d^{-}(v)=2 m$ para todo $v \in V(G)$. Para cada $v \in V(G)$, decompomos o conjunto das arestas que saem de $v$ em $m$ caminhos de comprimento 2. Seja $\mathcal{B}$ a 2-decomposição composta por um rastro qualquer de cada um desses 2-caminhos, e note que $\mathcal{B}(v)=d^{-}(v)=2 m$ para todo vértice $v$ de $V(G)$. Isso conclui a prova. 
O teorema a seguir é o nosso resultado principal sobre decomposição em caminhos de comprimento ímpar.

Teorema 4.10. Sejam $\ell, g$ e $m$ inteiros positivos tais que $\ell$ é impar e $g \geq 3$, e seja $G$ um grafo $m \ell$-regular com cintura pelo menos g e que contém um $m$-fator. Se $m>2\lfloor(\ell-2) /(g-2)\rfloor$, então $G$ admite uma $\ell$-decomposição em caminhos balanceada (e, consequentemente, uma $P_{\ell}$-decomposição balanceada).

Demonstração. A prova é por indução em $\ell$. Pelo Teorema 4.8, o resultado vale para $\ell=3$ e $g \geq 3$. Fixe $\ell \geq 5$ e suponha que o resultado seja válido para $\ell-2$.

Seja $M$ um $m$-fator de $G$. O grafo $G-E(M)$ é $m(\ell-1)$-regular e, portanto, pelo Teorema 2.1, admite uma decomposição em 2-fatores $\left\{F_{1}, \ldots, F_{m(\ell-1) / 2}\right\}$. Seja $H$ a união de $m$ desses fatores. Então $H$ é um $2 m$-fator de $G$ e $G^{\prime}=G-E(H)$ é um grafo $m(\ell-2)$-regular com cintura pelo menos $g$. Note que, $m>2\lfloor(\ell-2) /(g-2)\rfloor \geq 2\lfloor(\ell-4) /(g-2)\rfloor$. Portanto, pela hipótese de indução, $G^{\prime}$ admite uma $(\ell-2)$-decomposição em caminhos balanceada $\mathcal{B}^{\prime}$.

Afirmamos que $\mathcal{B}^{\prime}(v)=m$ para todo vértice $v$ em $V(G)$. Seja $|V(G)|=n$. Note que $G^{\prime}$ contém $n m(\ell-2) / 2$ arestas e, consequentemente, $\mathcal{B}^{\prime}$ contém $n m / 2$ caminhos. Logo, $\sum_{v \in V(G)} \mathcal{B}^{\prime}(v)=n m$. Como $\mathcal{B}^{\prime}$ é balanceada, $\mathcal{B}^{\prime}(v)=m$ para todo vértice $v$ de $G$ (veja Fato 4.2). Escolha uma orientação Euleriana para $H$. Note que $d_{H}^{+}(v)=m=\mathcal{B}^{\prime}(v)$. Portanto, para cada rastro $B^{\prime}=x_{1} \cdots x_{\ell-1}$ em $\mathcal{B}^{\prime}$ podemos escolher arestas $x_{1} x_{0}$ e $x_{\ell-1} x_{\ell}$ de $H$ que saem de $x_{1}$ e $x_{\ell-1}$, respectivamente, e adicioná-las a $B$, construindo o $\ell$-rastro $B=x_{0} x_{1} \cdots x_{\ell-1} x_{\ell}$. Como $d_{H}^{+}(v)=m=\mathcal{B}^{\prime}(v)$, podemos fazer essa operação de forma a usar cada aresta de $H$ exatamente uma vez. Seja $\mathcal{B}$ a $\ell$-decomposição obtida. Observe que as arestas $x_{1} x_{0}$ e $x_{\ell-1} x_{\ell}$ são arestas quase-pendentes, respectivamente, em $x_{1}$ e $x_{\ell-1}$ na decomposição $\mathcal{B}$. Além disso, $\mathcal{B}(v)=d_{H}^{-}(v)=m$ para todo vértice $v$ em $V(G)$, porque $H$ tem uma orientação Euleriana. Portanto, $\mathcal{B}$ é balanceada. Tome $r=\lfloor(\ell-2) /(g-2)\rfloor$ e $k=m-r-1$. Note que $k \geq\lfloor(\ell-2) /(g-2)\rfloor$. Pela definição de aresta quase-pendente, as arestas de $H$ que saem de um vértice fixo $v$ de $G$ são precisamente as arestas quase-pendentes em $v$ na decomposição $\mathcal{B}$; consequentemente, $\mathcal{B}$ é $(m-1)$-pré-completa, i.e, $(k+r)$-pré-completa. Pelo Lema 4.5 aplicado com $g, k, \ell$, e $r$, o grafo $G$ admite uma $\ell$-decomposição $k$-completa e balanceada $\mathcal{B}^{*}$. Como $k \geq$ $\lfloor(\ell-2) /(g-2)\rfloor$, pelo Lema 4.7, $G$ admite uma $\ell$-decomposição em caminhos balanceada e $k$ completa.

A prova do Teorema 4.11 é similar à prova do Teorema 4.10. Observe que no Teorema 4.11 não é necessária a hipótese da existência de um $m$-fator.

Teorema 4.11. Sejam $\ell, g$ e $m$ inteiros positivos tais que $g \geq 3$ e seja $G$ um grafo $2 m \ell$-regular com cintura pelo menos $g$. Se $m>\lfloor(\ell-2) /(g-2)\rfloor$, então $G$ admite uma $\ell$-decomposição em caminhos balanceada (e, consequentemente, uma $P_{\ell}$-decomposição balanceada).

Demonstração. A prova é por indução em $\ell$. Pela Proposição 4.9 , o resultado vale para $\ell=2$ e $g \geq 3$. Pelo Teorema 4.8, o resultado vale para $\ell=3$ e $g \geq 3$ (note que o Teorema 2.1 garante que $G$ contém um $2 m$-fator). Fixe $\ell \geq 4$ e suponha que o resultado seja válido para $\ell-2$.

Como $G$ é $2 m \ell$-regular, o Teorema 2.1 garante que $G$ admite uma decomposição em 2-fatores $\left\{F_{1}, \ldots, F_{m \ell}\right\}$. Seja $H$ a união de $2 m$ desses fatores. Então $H$ é um $4 m$-fator de $G$ e $G^{\prime}=G-E(H)$ é um grafo $2 m(\ell-2)$-regular com cintura pelo menos $g$. Note que, $m>\lfloor(\ell-2) /(g-2)\rfloor \geq$ 
$\lfloor(\ell-4) /(g-2)\rfloor$. Portanto, pela hipótese de indução, $G^{\prime}$ admite uma $(\ell-2)$-decomposição em caminhos balanceada $\mathcal{B}^{\prime}$.

Analogamente à prova do Teorema 4.10, podemos estender os $(\ell-2)$-rastros em $\mathcal{B}^{\prime}$ para $\ell$ rastros, obtendo uma $\ell$-decomposição balanceada $\mathcal{B}$. Aqui, temos que $\mathcal{B}$ é $(2 m-1)$-pré-completa. Tome $r=\lfloor(\ell-2) /(g-2)\rfloor$ e $k=2 m-r-1$. Note que $k \geq\lfloor(\ell-2) /(g-2)\rfloor$. Pelo Lema 4.5 aplicado com $g, k$, $\ell$, e $r$, o grafo $G$ admite uma $\ell$-decomposição $k$-completa e balanceada $\mathcal{B}^{*}$. Como $k \geq\lfloor(\ell-2) /(g-2)\rfloor$, pelo Lema 4.7, $G$ admite uma $\ell$-decomposição em caminhos balanceada e $k$-completa.

\subsection{Melhorias para grafos regulares ímpares com cintura pelo menos $\ell-1$ e grafos bipartidos}

Seja $\ell$ um inteiro positivo ímpar. Se considerarmos um grafo $m \ell$-regular $G$ com cintura pelo menos $\ell-1$, então o Teorema 4.10 garante que $G$ admite uma decomposição em caminhos de comprimento $\ell$, para todo inteiro $m \geq 3$. Nesta seção provamos que esse resultado também vale para $m \leq 2$. Além disso, mostramos como melhorar, quando $G$ é bipartido, as cotas inferiores para $m$ dadas nos Teoremas 4.10 e 4.11 .

\subsubsection{Decomposições de grafos $m \ell$-regulares com cintura pelo menos $\ell-1$}

Nesta subseção mostramos que o enunciado do Teorema 4.10 permanece válido quando $m=1$ ou $m=2$. O caso $m=2$ é consequência do seguinte resultado obtido por Kouider e Lonc [KL99].

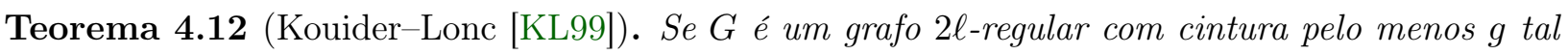
que $\ell \leq 2 g-3$, então $G$ admite uma $P_{\ell}$-decomposição balanceada.

Para o caso $m=1$, generalizamos o resultado em [BMW15], que afirma que todo grafo 5-regular livre de triângulos e que contém um emparelhamento perfeito admite uma $P_{5}$-decomposição.

Teorema 4.13. Seja l um inteiro positivo impar. Se $G$ é um grafo $\ell$-regular com cintura pelo menos $\ell-1$ e que contém um emparelhamento perfeito, então $G$ admite uma $\ell$-decomposição em caminhos (e, consequentemente, uma $P_{\ell}$-decomposição).

Observamos que para $\ell=3$, circuitos de todos os comprimentos possíveis são permitidos em $G$. A prova do Teorema 4.13 é muito similar à prova em [BMW15] e, portanto, a prova apresentada aqui simplificará alguns detalhes. Primeiramente, precisamos apresentar algumas definições.

Seja $\mathcal{B}$ uma $\ell$-decomposição de um grafo $\ell$-regular ímpar $G$ com cintura pelo menos $\ell-1$. Pelo Fato 4.2, toda decomposição em $\ell$-trilhas de um grafo $\ell$-regular é balanceada. Uma vez que $\mathcal{B}$ é uma decomposição em rastros, nenhum elemento de $\mathcal{B}$ é um circuito, caso contrário, teríamos $\mathcal{B}(v) \geq 3$ para algum vértice $v$ de $G$. Logo, se $T=x_{0} \cdots x_{\ell}$ é um elemento de $\mathcal{B}$ que não induz um caminho, então $x_{\ell}=x_{1}$, e dizemos que $x_{2}$ é o vértice de conexão de $T$. Uma decomposição $\mathcal{B}$ é dita boa se valem as seguintes duas propriedades: (i) existe exatamente uma aresta quase-pendente em cada vértice $v \in V(G)$ que é vértice de conexão de algum elemento de $\mathcal{B}$; (ii) cada vértice de $G$ é o vértice 
de conexão de no máximo um elemento de $\mathcal{B}$. Denotamos por $\tau(\mathcal{D})$ o número de elementos de $\mathcal{B}$ que não induzem caminhos em $G$ (note que essa definição coincide com a definição de $\tau$ dada na Seção 4.1).

Prova do Teorema 4.13. Seja $G$ como na hipótese, e seja $M$ um emparelhamento perfeito de $G$. Uma vez que o caso $\ell=3$ é provado em [BF83, Kot57] (e também no começo deste capítulo), vamos supor que $\ell \geq 5$ e provar o resultado por indução em $\ell$. O grafo $G-M$ é $(\ell-1)$-regular e, pelo Teorema 2.1, contém dois 2-fatores $F_{1}$ e $F_{2}$. Logo, $G^{\prime \prime}=G-E\left(F_{1}\right)-E\left(F_{2}\right)$ é um grafo $(\ell-4)$-regular com cintura pelo menos $\ell-1$, e, pela hipótese de indução, $G^{\prime \prime}$ admite uma $(\ell-4)$-decomposição balanceada em caminhos $\mathcal{B}^{\prime \prime}$. Considere uma orientação Euleriana das arestas em $F_{1}$. Adicionando uma aresta de $F_{1}$ em cada vértice final de cada elemento de de $\mathcal{B}^{\prime \prime}$, estendemos os $(\ell-4)$-rastros em $\mathcal{B}^{\prime \prime}$ para $(\ell-2)$-rastros peculiares, obtendo uma $(\ell-2)$-decomposição em caminhos balanceada $\mathcal{B}^{\prime}$. Agora, considere uma orientação Euleriana das arestas em $F_{2}$. Podemos estender os $(\ell-2)$-rastros em $\mathcal{B}^{\prime}$ para $\ell$-rastros peculiares, e obtemos uma $\ell$-decomposição $\mathcal{B}$ (dessa vez podemos obter elementos que não induzem caminhos). Como $\mathcal{B}^{\prime}(v)=1$ para todo $v \in V(G)$, existe exatamente uma aresta quase-pendente em cada vértice $v \in V(G)$ na decomposição $\mathcal{B}$. Mais especificamente, a aresta de $F_{2}$ que sai do vértice $v$ é precisamente a aresta quase-pendente em $v$ na decomposição $\mathcal{B}$.

Seja $v$ um vértice de $G$. Como $\mathcal{B}^{\prime \prime}(v)=1$, temos que $v$ pode ser o vértice de conexão de no máximo um elemento de $\mathcal{B}$. Logo, $\mathcal{B}$ é uma decomposição em $\ell$-rastros peculiares boa. Então podemos escolher $\mathcal{B}^{*}$ como sendo uma decomposição em $\ell$-rastros peculiares boa que minimiza $\tau\left(\mathcal{B}^{*}\right)$. Se $\tau\left(\mathcal{B}^{*}\right)=0$, então $\mathcal{B}^{*}$ é uma $\ell$-decomposição em caminhos, e a prova está terminada. Logo, podemos supor por contradição que $\tau\left(\mathcal{B}^{*}\right)>0$.

Seja $T=x_{0} \cdots x_{\ell}$ um elemento de $\mathcal{B}^{*}$ que não induz um caminho. Seja $Q$ o elemento de $\mathcal{B}^{*}$ que contém a (única) aresta quase-pendente em $x_{2}$. Dizemos que o par $(T, Q)$ é um casal de $\mathcal{B}^{*}$. Vamos considerar um digrafo auxiliar $H$ da seguinte forma. Os vértices de $H$ são os elementos de $\mathcal{B}$; e para cada casal $(T, Q)$ de $\mathcal{B}$ colocamos um arco de $T$ para $Q$. Portanto, se $T$ induz um caminho, então $d_{H}^{+}(T)=0$, caso contrário, temos que $d_{H}^{+}(T)=1$. Afirmamos que $d_{H}^{+}(T)-d_{H}^{-}(T) \leq 0$ para todo $T$ em $\mathcal{B}$. Suponha, por contradição, que exista um elemento $T$ em $\mathcal{B}$ tal que $d_{H}^{+}(T)-d_{H}^{-}(T)=1$. Como $T$ não induz um caminho, sejam $T=x_{0} \cdots x_{\ell}$ e $Q=y_{0} \cdots y_{\ell}$, tais que $(T, Q)$ é um casal e $y_{1} y_{0}$ é a aresta quase-pendente em $y_{1}=x_{2}$. Tome novos elementos $T^{\prime}, Q^{\prime}$ tais que $\bar{T}^{\prime}=\bar{T}-x_{2} x_{1}+x_{2} y_{0}$ e $\bar{Q}^{\prime}=\bar{Q}-y_{1} y_{0}+y_{1} x_{1}$, e considere a decomposição $\mathcal{B}^{+}=\mathcal{B}^{*}-T-Q+T^{\prime}+Q^{\prime}$. Se $y_{0} \in V(T)$, então $T^{\prime}$ contém um circuito de comprimento menor que $\ell-1$. Logo, $T^{\prime}$ induz um caminho e a aresta $x_{\ell-1} x_{\ell}$ (que é quase-pendente em $x_{\ell-1}$ na decomposição $\mathcal{B}^{*}$ ) não é quase-pendente na decomposição $\mathcal{B}^{+}$. Logo, $\mathcal{B}^{+}$é uma decomposição em $\ell$-rastros peculiares boa de $G$ tal que $\tau\left(\mathcal{B}^{+}\right) \leq$ $\tau\left(\mathcal{B}^{*}\right)-1$, uma contradição à minimalidade de $\tau\left(\mathcal{B}^{*}\right)$. Portanto, $d_{H}^{+}(T)-d_{H}^{-}(T) \leq 0$ para todo $T$ em $\mathcal{B}^{*}$. Como $\sum_{T \in \mathcal{B}^{*}} d_{H}^{+}(T)=\sum_{T \in \mathcal{B}^{*}} d_{H}^{-}(T)$, temos que $d_{H}^{+}(T)-d_{H}^{-}(T)=0$ para todo $T \in \mathcal{B}^{*}$, e consequentemente $H$ consiste de circuitos direcionados vértice-disjuntos (e vértices isolados, que representam rastros de $\mathcal{B}^{*}$ que induzem caminhos em $G$ ).

Agora precisamos separar os casais em dois tipos. Seja $(T, Q)$ um casal, onde $T=x_{0} \cdots x_{\ell}$, $Q=y_{0} \cdots y_{\ell}, \operatorname{com} x_{\ell}=x_{1}$ e $y_{\ell}=y_{1}$. Note que $x_{2}$ é o vértice de conexão de $T$. Dizemos que $(T, Q)$ é do tipo 1 se $y_{1}=x_{2}$; e do tipo 2 se $y_{\ell-1}=x_{2}$ (veja Figura 4.5).

Seja $T_{0} \cdots T_{k-1}$ um circuito de elementos de $\mathcal{B}^{*}$, onde $\left(T_{i}, T_{i+1}\right)$ é um casal de $\mathcal{B}^{*}$, para $i=$ 

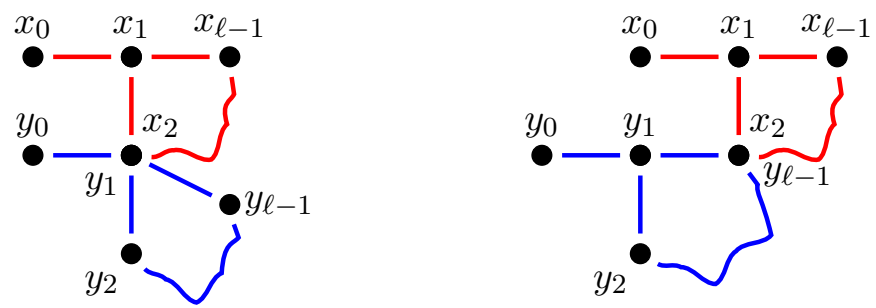

Figura 4.5: Casais do tipo 1 e 2 .

$0, \ldots, k-1$ (tomando índices módulo $k$ ). Suponha que $\left(T_{k-1}, T_{0}\right)$ seja do tipo 1 , e seja $T_{0}=x_{0} \cdots x_{\ell}$, $T_{1}=y_{0} \cdots y_{\ell}$, onde $x_{2} \in\left\{y_{1}, y_{\ell-1}\right\}$. Tome $T_{0}^{\prime}$ e $T_{1}^{\prime}$ tais que $\bar{T}_{0}^{\prime}=\bar{T}_{0}-x_{2} x_{1}+e$ e $\bar{T}_{1}^{\prime}=\bar{T}_{1}-e+x_{2} x_{1}$, onde $e$ é a aresta de $T_{1}$ quase-pendente em $x_{2}$ na decomposição $\mathcal{B}^{*}$ (veja Figura 4.6a-b).

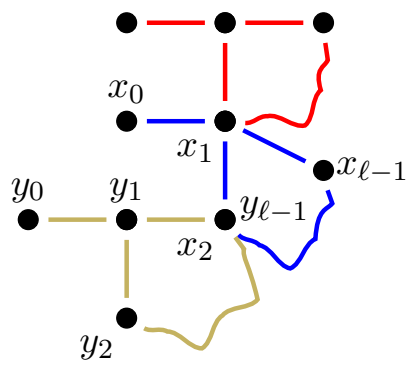

(a)

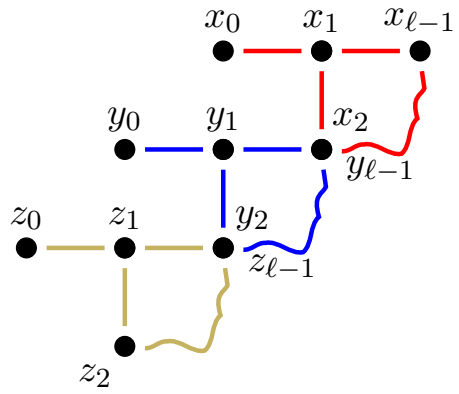

(c)

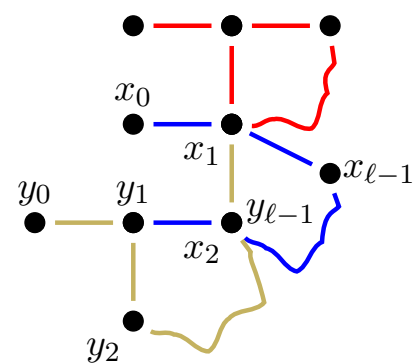

(b)

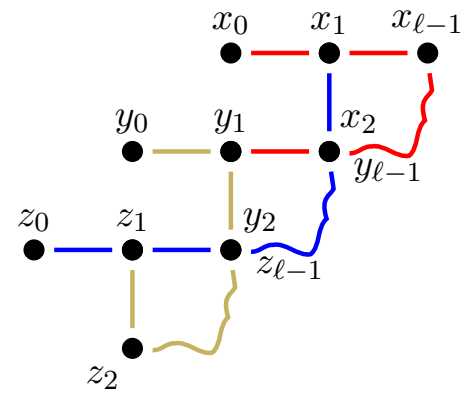

(d)

Figura 4.6: Três elementos consecutivos em circuitos com número ímpar de elementos.

Temos que $\mathcal{B}^{+}=\mathcal{B}^{*}-T_{0}-T_{1}+T_{0}^{\prime}+T_{1}^{\prime}$ é uma decomposição em $\ell$-rastros peculiares boa de $G$ tal que $\tau\left(\mathcal{B}^{+}\right) \leq \tau\left(\mathcal{B}^{*}\right)-1$, uma contradição à minimalidade de $\tau\left(\mathcal{B}^{*}\right)$. Assim, podemos assumir que $\left(T_{i}, T_{i+1}\right)$ é do tipo 2 para todo $i=0, \ldots, k-1$. Se $k$ é par, então fazemos a troca de arestas entre $T_{2 i-1}$ e $T_{2 i}$ para $i=0, \ldots, k / 2-1$ da seguinte forma: tome $T_{2 i-1}=x_{0} \cdots x_{\ell}$, $T_{2 i}=y_{0} \cdots y_{\ell}$, onde $y_{\ell}=y_{1}$ e $y_{\ell-1}=x_{2}$, e considere $T_{2 i-1}^{\prime}$ e $T_{2 i}^{\prime}$ tal que $\bar{T}_{2 i-1}^{\prime}=\bar{T}_{2 i-1}-x_{2} x_{1}+y_{\ell-1} y_{\ell}$ e $\bar{T}_{2 i}^{\prime}=\bar{T}_{2 i}-y_{\ell-1} y_{\ell}+x_{2} x_{1}$. Agora suponha que $k$ seja ímpar. Seja $T_{0}=x_{0} \cdots x_{\ell}, T_{1}=y_{0} \cdots y_{\ell}$, e $T_{2}=z_{0} \cdots z_{\ell}$, onde $x_{\ell}=x_{1}, y_{\ell}=y_{1}, z_{\ell}=z_{1}$, e $y_{\ell-1}=x_{2}, z_{\ell-1}=y_{2}$. Tome $T_{i}^{\prime}$ para $i=0,1,2$ tal que $\bar{T}_{0}^{\prime}=\bar{T}_{0}-x_{2} x_{1}+y_{\ell-1} y_{\ell}, \bar{T}_{1}^{\prime}=\bar{T}_{1}-y_{\ell-1} y_{\ell}-y_{2} y_{1}-y_{1} y_{0}+x_{2} x_{1}+z_{\ell-1} z_{\ell}+z_{1} z_{0}$, e $\bar{T}_{2}^{\prime}=$ $\bar{T}_{2}-z_{\ell-1} z_{\ell}-z_{1} z_{0}+y_{2} y_{1}+y_{1} y_{0}$ (veja Figura 4.6c-d). Além disso, troque as arestas entre $T_{2 i}$ e $T_{2 i+1}$ analogamente ao caso par, para $i=2, \ldots,(k-1) / 2$. Portanto, $\mathcal{B}^{+}=\mathcal{B}^{*}-T_{0}-\cdots T_{k}+T_{0}^{\prime}+\cdots T_{k}^{\prime}$ é uma decomposição em $\ell$-rastros peculiares boa de $G$ tal que $\tau\left(\mathcal{B}^{+}\right)<\tau\left(\mathcal{B}^{*}\right)$. Em todos os casos obtivemos uma contradição à minimalidade de $\tau\left(\mathcal{B}^{*}\right)$. Isso conclui a prova. 


\subsubsection{Decomposições de grafos bipartidos}

Nessa subseção mostramos que para grafos bipartidos, podemos conseguir cotas melhores para os graus dos vértices. Para isso, usamos versões dos Lemas 4.5, 4.6, e 4.7 que foram provadas em [BMOWb]. Os seguintes lemas também serão usados no Capítulo 5.

Lema 4.14. Sejam $k$, $\ell$ e $r$ inteiros positivos, e $G$ um grafo bipartido. Se $r \geq\lfloor\ell / 2\rfloor$ e $G$ admite uma $\ell$-decomposição $(k+r)$-pré-completa $\mathcal{B}$, então $G$ admite uma $\ell$-decomposição $k$-completa $\mathcal{B}^{\prime}$ tal que $\mathcal{B}^{\prime}(v)=\mathcal{B}(v)$ para todo vértice $v$ de $G$.

Demonstração. Sejam $k, \ell, r, G=(A, B ; E)$ e $\mathcal{B}$ como na hipótese do lema. Seja $\mathcal{B}^{\prime}$ uma $\ell$-decomposição $(k+r)$-pré-completa de $G$ tal que $\mathcal{B}^{\prime}(v)=\mathcal{B}(v)$ para todo vértice $v$ de $G$, e que maximiza o somatório $\sum_{v \in V(G)} \operatorname{Pend}\left(v, \mathcal{B}^{\prime}\right)$. Afirmamos que $\mathcal{B}^{\prime}$ é $k$-completa, i.e, $\operatorname{Pend}\left(v, \mathcal{B}^{\prime}\right)>k$ para cada vértice $v$ de $G$. Suponha, por contradição, que $\mathcal{B}^{\prime}$ não seja $k$-completa. Então existe um vértice $u$ (sem perda de generalidade) em $A$ tal que $\operatorname{Pend}\left(u, \mathcal{B}^{\prime}\right) \leq k$. Logo, existem pelo menos $r+1$ arestas quase-pendentes em $u$ que não são pendentes em $u$, digamos $u x_{1}, \ldots, u x_{r+1}$. Seja $T_{1}=y_{0} y_{1} \cdots, y_{\ell}$ o elemento de $\mathcal{B}^{\prime}$ que contém $u x_{1}$, onde, sem perda de generalidade, $y_{0}=x_{1}$ e $y_{1}=u$. Os vértices de $T_{1}$ em $B$ são $y_{0}, y_{2}, \ldots, y_{2\lfloor\ell / 2\rfloor}$. Como $y_{1} y_{0}$ não é aresta pendente, $y_{0} \in\left\{y_{2}, \ldots, y_{2\lfloor\ell / 2\rfloor}\right\}$. Logo, o número de vértices de $T_{1}$ em $B$ é no máximo $\lfloor\ell / 2\rfloor$. Como $r \geq\lfloor\ell / 2\rfloor$, existe pelo menos uma aresta quase-pendente que não é pendente, digamos $u x_{i}$, em $u$ na decomposição $\mathcal{B}^{\prime}$ tal que $x_{i} \notin V\left(T_{1}\right)$. Seja $T_{i}=z_{0} z_{1} \cdots z_{\ell}$ o elemento de $\mathcal{B}^{\prime}$ que contém $u x_{i}$, onde $z_{0}=x_{i}$ e $z_{1}=u$. Tome $T_{1}^{\prime}=z_{0} y_{1} y_{2} \cdots y_{\ell}$ e $T_{i}^{\prime}=y_{0} z_{1} z_{2} \cdots z_{\ell}$, e ponha $\mathcal{B}^{\prime \prime}=\mathcal{B}^{\prime}-T_{1}-T_{i}+T_{1}^{\prime}+T_{i}^{\prime}$. Note que as arestas quase-pendentes em $\mathcal{B}^{\prime \prime}$ são as mesmas em $\mathcal{B}^{\prime}$, porém, a aresta $u x_{i}$ é aresta pendente em $\mathcal{B}^{\prime \prime}$. Assim, concluímos que $\mathcal{B}^{\prime \prime}(v)=\mathcal{B}^{\prime}(v)$ para todo vértice $v$ de $G$. Além disso, temos que $\operatorname{Pend}\left(u, \mathcal{B}^{\prime \prime}\right) \geq \operatorname{Pend}\left(u, \mathcal{B}^{\prime}\right)+1$, e $\operatorname{Pend}\left(v, \mathcal{B}^{\prime \prime}\right)=\operatorname{Pend}\left(v, \mathcal{B}^{\prime}\right)$ para todo vértice $v$ em $V(G)-u$. Portanto, $\sum_{v \in V(G)} \operatorname{Pend}\left(v, \mathcal{B}^{\prime \prime}\right)>$ $\sum_{v \in V(G)} \operatorname{Pend}\left(v, \mathcal{B}^{\prime}\right)$, uma contradição à maximalidade do somatório $\sum_{v \in V(G)} \operatorname{Pend}\left(v, \mathcal{B}^{\prime}\right)$. Logo, $\mathcal{B}^{\prime}$ é $k$-completa.

Lema 4.15. Sejam $\ell$ e $k$ inteiros positivos e $G$ um grafo bipartido. Se $k \geq\lceil\ell / 2\rceil$ e $\mathcal{B}$ é uma $\ell$-decomposição k-completa de $G$, então $\mathcal{B}$ é viável.

Demonstração. Sejam $\ell, k, G$ e $\mathcal{B}$ como na hipótese do lema. Fixe $v \in V(G)$, e seja $T$ uma $\ell$-trilha de $G$ contendo um circuito $C$ tal que $v \in V(C)$. Uma vez que $\mathcal{B}$ é $k$-completa, $\operatorname{Pend}(v, \mathcal{B})>k$. Sejam $v w_{1}, \ldots, v w_{k+1}$ as arestas pendentes em $v$ na decomposição $\mathcal{B}$. Afirmamos que existe um índice $1 \leq i \leq k+1$ tal que $w_{i} \notin V(T)$. Seja $W=\left\{w_{1}, \ldots, w_{k+1}\right\}$. Seja $G=(A, B ; E)$ e suponha, sem perda de generalidade, que $v \in A$. Como $G$ é bipartido, $W \subset B$. Além disso, como $T$ contém um circuito, $T$ contém no máximo $\ell$ vértices, $|V(T) \cap B| \leq\lceil\ell / 2\rceil \leq k$. Mas como $|W|=k+1$, concluímos que existe um vértice $w \in W$ tal que $w \notin V(T)$.

Para desemaranhar os rastros de uma $\ell$-decomposição $\mathcal{B}$ de um grafo bipartido $G$ precisamos que $\mathcal{B}$ seja $\lceil\ell / 2\rceil$-completa. A prova do próximo resultado é análoga à prova do Lema 4.7 , usando o Lema 4.15 no lugar do Lema 4.6.

Lema 4.16. Sejam $k$, $\ell$ inteiros positivos e seja $G$ um grafo bipartido. Se $k \geq\lceil\ell / 2\rceil$ e $\mathcal{B}$ é uma $\ell$-decomposição $k$-completa de $G$, então $G$ admite uma $\ell$-decomposição em caminhos $k$-completa $\mathcal{B}^{\prime}$ tal que $\mathcal{B}^{\prime}(v)=\mathcal{B}(v)$ para todo vértice $v$ de $G$. 
Os próximos dois teoremas são os resultados principais desta seção. Similarmente ao caso de grafos regulares com cintura prescrita, a prova para caminhos de comprimento par é muito similar à prova para caminhos de comprimento ímpar. Aqui mostramos apenas a prova para decomposições em caminhos de comprimento ímpar.

Teorema 4.17. Sejam $\ell$ e $m$ inteiros positivos tais que $\ell$ é ímpar, e seja $G$ um grafo bipartido $m \ell$-regular. Se $m>\ell$, então $G$ admite uma $\ell$-decomposição em caminhos balanceada (e, consequentemente, uma $P_{\ell}$-decomposição balanceada).

Teorema 4.18. Sejam $\ell$ e $m$ inteiros positivos e seja $G$ um grafo bipartido $2 m \ell$-regular. Se $m>\ell / 2$, então $G$ admite uma $\ell$-decomposição em caminhos balanceada (e, consequentemente, uma $P_{\ell}$-decomposição balanceada).

Prova do Teorema 4.17. Como todo grafo regular bipartido admite uma decomposição em 1-fatores, o resultado vale para $\ell=1$. Fixe $\ell \geq 3$ e suponha que o enunciado seja verdadeiro para $\ell-2$. Seja $\left\{M_{1}, \ldots, M_{m \ell}\right\}$ uma 1-fatorização de $G$. Seja $H$ o grafo obtido da união de $2 m$ desses 1 -fatores, e considere $G^{\prime}=G-E(H)$. O grafo $G^{\prime}$ é um grafo $m(\ell-2)$-regular. Como $m>\ell / 2>(\ell-2) / 2$, pela hipótese de indução, $G^{\prime}$ admite uma $(\ell-2)$-decomposição em caminhos balanceada $\mathcal{B}^{\prime}$.

Pelo Fato 4.2 , temos que $\mathcal{B}^{\prime}(v)=m$ para todo vértice $v$ em $V(G)$. Tome uma orientação Euleriana para $H$. Note que $d_{H}^{+}(v)=m=\mathcal{B}^{\prime}(v)$. Logo, podemos estender cada rastro $P$ de $\mathcal{B}^{\prime}$ para um rastro peculiar $T$ adicionando a $P$ uma aresta saindo de cada um de seus vértices finais. Seja $\mathcal{B}$ a $\ell$-decomposição obtida, e note que $\mathcal{B}(v)=d_{H}^{-}(v)=m$ para todo vértice $v$ em $V(G)$. Portanto, $\mathcal{B}$ é balanceada. Tome $r=(\ell-1) / 2$ e $k=m-r-1$, e note que $k \geq(\ell+1) / 2$. Pela definição de aresta quase-pendente, as arestas de $H$ que saem de um vértice fixado $v$ de $G$ são precisamente as arestas quase-pendentes em $v$ na decomposição $\mathcal{B}$; consequentemente, $\mathcal{B}$ é $(m-1)$-pré-completa. Pelo Lema 4.14 aplicado com $k$, $\ell$, e $r$, o grafo $G$ admite uma $\ell$-decomposição balanceada e $k$-completa $\mathcal{B}^{*}$. Como $k \geq(\ell+1) / 2$, pelo Lema 4.16, $G$ admite uma $\ell$-decomposição em caminhos balanceada e $k$-completa.

O seguinte teorema sumariza os resultados apresentados neste capítulo.

Teorema 4.19. Sejam $\ell, g$ e $m$ inteiros positivos, onde $g \geq 3$. Então valem os seguintes resultados.

(1) Se l é impar,

a) Se $m>2\lfloor(\ell-2) /(g-2)\rfloor$ e $G$ é um grafo m $\ell$-regular com cintura pelo menos $g$ e contém um $m$-fator, então $G$ admite uma $P_{\ell}$-decomposição.

b) Se $g \geq \ell-1$ e $G$ é um grafo $m \ell$-regular com cintura pelo menos $g$ e contém um $m$-fator, então $G$ admite uma $P_{\ell}$-decomposição.

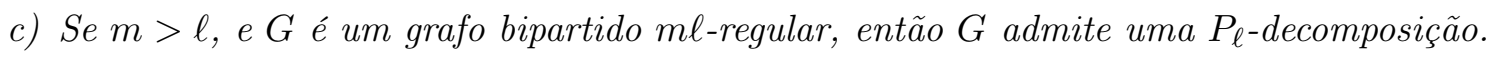

(2) Se $m>\lfloor(\ell-2) /(g-2)\rfloor$ e $G$ é um grafo $2 m \ell$-regular com cintura pelo menos $g$, então $G$ admite uma $P_{\ell^{-}}$decomposição.

(3) Se $m>\ell / 2$ e $G$ é um grafo bipartido $2 m \ell$-regular, então $G$ admite uma $P_{\ell}$-decomposição. 


\section{Capítulo 5}

\section{Grafos altamente conexos}

Neste capítulo abordamos o problema de decompor grafos altamente aresta-conexos em caminhos. Mais especificamente, atacamos a seguinte conjectura, proposta por Barát e Thomassen [BT06].

Conjectura 5.1 (Barát-Thomassen, 2006). Para cada árvore $T$, existe um inteiro positivo $k_{T}$ tal que, se $G$ é um grafo $k_{T}$-aresta-conexo e $|E(G)|$ é divisivel por $|E(T)|$, então $G$ admite uma T-decomposição.

Barát e Thomassen [BT06] provaram que no caso em que $T$ é a garra $K_{1,3}$, essa conjectura é equivalente à Conjectura Fraca de 3-fluxo de Tutte, proposta por Jaeger [Jae88]. Eles também observaram que a Conjectura 5.1 é falsa quando $T$ é um grafo que contém circuitos. Em uma série de artigos, Thomassen [Tho08a, Tho08b, Tho12, Tho13a, Tho13b] provou a validade da Conjectura 5.1 para estrelas, algumas biestrelas, caminhos de comprimento 3 , e caminhos de comprimento $2^{k}$, para todo inteiro positivo $k$. Provamos a Conjectura 5.1 para caminhos de comprimento 5 [BMOWa] e, mais recentemente, para caminhos de qualquer comprimento fixo [BMOWb]. Merker [Mer15] provou a Conjectura 5.1 para árvores com diâmetro 3 e 4, e algumas árvores de diâmetro 5, incluindo o caminho de comprimento 5. Utilizando uma técnica diferente, Bensmail, Harutyunyan, Le, e Thomassé [BHLT15] também provaram a Conjectura 5.1 para caminhos de comprimento fixo. Neste capítulo, apresentamos uma simplificação da prova apresentada em [BMOWb].

Primeiramente, consideramos um resultado provado por Barát e Gerbner [BG14] e, independentemente, por Thomassen [Tho13a], que diz que a Conjectura 5.1 é equivalente à seguinte versão para grafos bipartidos.

Conjectura 5.2. Para cada árvore $T$, existe um inteiro positivo $k_{T}^{\prime}$ tal que, se $G$ é um grafo bipartido $k_{T}^{\prime}$-aresta-conexo e $|E(G)|$ é divisivel por $|E(T)|$, então $G$ admite uma $T$-decomposição.

Mais espeficiamente esses autores provaram o seguinte teorema.

Teorema 5.3 (Barát-Gerbner [BG14]; Thomassen [Tho13a]). Seja T uma árvore com $t$ vértices, $t>4$. Os seguintes enunciados são equivalentes.

(i) Existe um inteiro positivo $k_{T}^{\prime}$ tal que, se $G$ é um grafo bipartido $k_{T}^{\prime}$-aresta-conexo e $|E(G)|$ é divisivel por $|E(T)|$, então $G$ admite uma T-decomposição.

(ii) Existe um inteiro positivo $k_{T}$ tal que, se $G$ é um grafo $k_{T}$-aresta-conexo e $|E(G)|$ é divisivel por $|E(T)|$, então $G$ admite uma T-decomposição. 
Além disso, $k_{T} \leq 4 k_{T}^{\prime}+16(t-1)^{6 t-5}$ e se $T$ tem diâmetro no máximo 3 , então $k_{T} \leq 4 k_{T}^{\prime}+16 t(t-1)$.

Para provar o Teorema 5.3, esses mesmos autores provaram o seguinte resultado.

Lema 5.4. Seja $T$ uma árvore com $t$ vértices, $t>4$, e seja $k$ um inteiro positvo. Se $G$ é $(4 k+$ $\left.16(t-1)^{6 t-5}\right)$-aresta-conexo, então $G$ pode ser decomposto em dois grafos $G_{1}$ e $G_{2}$ tais que

i) $G_{1}$ é bipartido e k-aresta-conexo;

ii) $G_{2}$ admite uma T-decomposição.

O Lema $5.4 \mathrm{diz}$, em outras palavras, que se $G$ é altamente aresta-conexo, então podemos remover cópias de $T$ de forma a obter um grafo bipartido ainda altamente aresta-conexo. Portanto, de agora em diante, vamos nos restringir ao caso em que o grafo $G$ é bipartido. O resultado principal desta seção, Corolário 5.35, diz que se $\ell$ é um inteiro positivo e $G$ é um grafo bipartido $2(13 \ell+4 r-4)$ aresta-conexo, onde $r=\max \{32(\ell-1), \ell(\ell+1)\}$, e $|E(G)|$ é divisível por $\ell$, então $G$ admite uma $P_{\ell}$-decomposição.

Assim como as provas do Capítulo 4, a prova do resultado principal deste capítulo consiste basicamente de um passo de fatorização e um passo de indução.

Este capítulo está organizado da seguinte forma. Na Seção 5.1, apresentamos alguns resultados que serão utilizados nas provas da Seção 5.2. Na Seção 5.2, adaptamos as definições de fatores e fatorizações para grafos bipartidos altamente aresta-conexos, definimos bifatorizações, e provamos uma versão do Teorema de Petersen (Teorema 2.1) para grafos altamente aresta-conexos. Na Seção 5.3, adaptamos a definição de decomposição balanceada para grafos que admitem bifatorizações, e utilizamos os Lemas 4.14 e 4.16 para decompor esses grafos em caminhos de comprimento fixo. $\mathrm{Na}$ Seção 5.4, unimos os resultados das Seções 5.2 e 5.3, e provamos o resultado principal dessa seção.

\subsection{Resultados preliminares}

Nesta seção definimos duas operações básicas sobre grafos: splitting de vértices (Subseção 5.1.1) e lifting de arestas (Subseção 5.1.2). Além disso, apresentamos resultados clássicos que relacionam essas operações com aresta-conexidade. Na Subseção 5.1.3, mostramos algumas propriedades de grafos altamente aresta-conexos que são utilizadas nas provas da Seção 5.2.

\subsubsection{Splitting de vértices}

Seja $G=(V, E)$ um grafo. Dado um vértice $v$ de $G$, um conjunto $S_{v}=\left\{d_{1}, \ldots, d_{s_{v}}\right\}$ de $s_{v}$ inteiros positivos é chamado uma sequência de subgraus para $v$ se $d_{1}+\cdots+d_{s_{v}}=d_{G}(v)$. Dizemos que um grafo $G^{\prime}$ é obtido por um $\left(v, S_{v}\right)$-splitting de $G$ se $G^{\prime}$ é composto de $G-v$ junto com $s_{v}$ novos vértices $v_{1}, \ldots, v_{s_{v}}$ e $d_{G}(v)$ novas arestas tais que $d_{G^{\prime}}\left(v_{i}\right)=d_{i}$, para $1 \leq i \leq s_{v}$, e $\bigcup_{i=1}^{s_{v}} N_{G^{\prime}}\left(v_{i}\right)=N_{G}(v)$.

Para um dado conjunto $V^{\prime}=\left\{v_{1}, \ldots, v_{r}\right\}$ de $r$ vértices de $G$, sejam $S_{v_{1}}, \ldots, S_{v_{r}}$ sequências de subgraus para $v_{1}, \ldots, v_{r}$, respectivamente. Sejam $H_{1}, \ldots, H_{r}$ grafos obtidos como se segue: $H_{1}$ é obtido a partir de $G$ por um $\left(v_{1}, S_{v_{1}}\right)$-splitting, o grafo $H_{2}$ é obtido a partir de $H_{1}$ por um $\left(v_{2}, S_{v_{2}}\right)$ splitting, e assim por diante, até $H_{r}$, que é obtido a partir de $H_{r-1}$ por um $\left(v_{r}, S_{v_{r}}\right)$-splitting. Dizemos que cada $H_{i}$ é um $\left\{S_{v_{1}}, \ldots, S_{v_{i}}\right\}$-destacamento de $G$. A grosso modo, um destacamento de $G$ é um grafo obtido por aplicações sucessivas de operações de splitting em vértices de $G$. Na Figura 5.1, o grafo $H$ é um $\left\{S_{a}, S_{e}\right\}$-destacamento de $G$, onde $S_{a}=\{2,3\}$ e $S_{e}=\{2,2,2\}$. O próximo 
resultado nos fornece condições suficientes para a existência de um destacamento $2 k$-aresta-conexo de um grafo $2 k$-aresta-conexo.

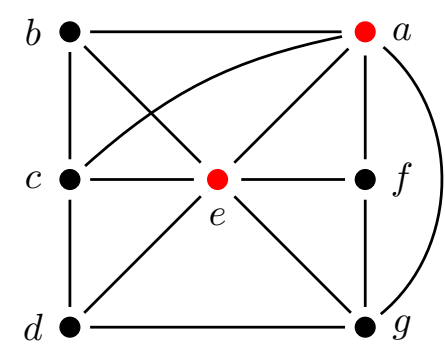

$G$

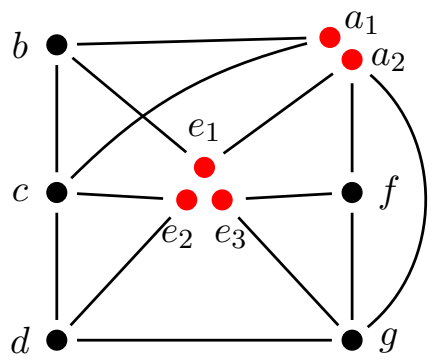

$H$

Figura 5.1: Um grafo $G$ e um grafo $H$ que é um $\left\{S_{a}, S_{e}\right\}$-destacamento de $G$.

Lema 5.5 (Nash-Williams [NW85]). Seja $G$ um grafo 2k-aresta-conexo, com $k \geq 1$ e $V(G)=$ $\left\{v_{1}, \ldots, v_{n}\right\}$. Para cada $v \in V(G)$, seja $S_{v}=\left\{d_{1}^{v}, \ldots, d_{s_{v}}^{v}\right\}$ uma sequência de subgraus para $v$ tal que $d_{i}^{v} \geq 2 k$ para $i=1, \ldots, s_{v}$. Então existe um $\left\{S_{v_{1}}, \ldots, S_{v_{n}}\right\}$-destacamento $2 k$-aresta-conexo de $G$.

\subsubsection{Lifting de arestas}

Sejam $G=(V, E)$ um grafo e $u, v, w$ vértices distintos de $G$ tais que $u v, v w \in E$. O multigrafo $G^{\prime}=(V,(E \backslash\{u v, v w\}) \cup\{u w\})$ é chamado de um uw-lifting (ou, simplesmente, um lifting) em $v$ (veja Figura 5.2). Note que $G^{\prime}$ pode conter arestas paralelas conectando $u$ e $w$. Se para todos pares distintos $x, y \in V \backslash\{v\}$, o número máximo de caminhos aresta-disjuntos ligando $x$ e $y$ em $G^{\prime}$ é o mesmo que em $G$, então o lifting em $v$ é chamado de admissivel. Se $v$ é um vértice de grau 2, então o lifting em $v$ é sempre admissível. Tal lifting, juntamente com a remoção de $v$ é chamado de supressão de $v$. O próximo resultado é conhecido como o Teorema de Lifting de Mader.

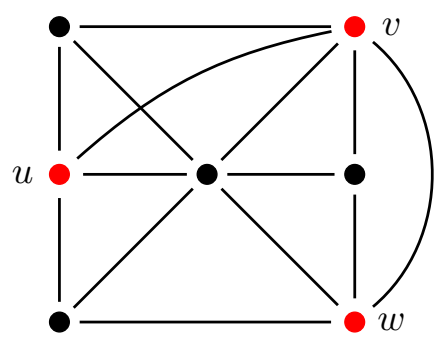

$G$

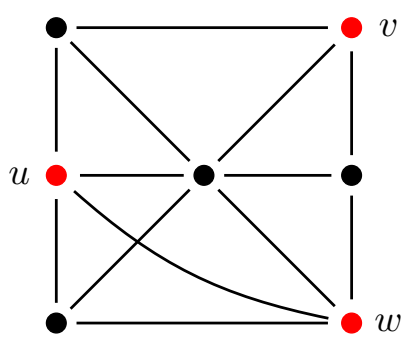

$G$

Figura 5.2: Um grafo $G$ e um grafo $H$ que é um uw-lifting de $G$.

Teorema 5.6 (Mader [Mad78]). Sejam G um multigrafo e v um vértice de G. Se v não é um vértice-de-corte, $d_{G}(v) \geq 4$, e $v$ tem pelo menos dois vizinhos, então existe um lifting admissivel em $v$.

O seguinte lema será útil na aplicação do Teorema de Lifting de Mader. Neste lema e em seguida, denotamos por $p_{G}(x, y)$ o número máximo de caminhos dois-a-dois aresta-disjuntos ligando os vértices $x$ e $y$ no grafo $G$. 
Lema 5.7. Sejam $G$ um multigrafo e $k$ um inteiro positivo. Se vé um vértice em $G$ tal que $d(v)<2 k$ e $p_{G}(x, y) \geq k$ para quaisquer dois vizinhos distintos $x$ e $y$ de $v$, então $v$ não é um vértice-de-corte.

Demonstração. Sejam $G, k$ e $v$ como no enunciado. Suponha, por contradição, que $v$ seja um vértice-de-corte, e considere os vértices $x$ e $y$ vizinhos de $v$ em diferentes componentes de $G-v$. Todo caminho ligando $x$ a $y$ contém $v$ como vértice interno. Como $p_{G}(x, y) \geq k$, então existem caminhos $P_{1}, \ldots, P_{k}$ dois-a-dois aresta-disjuntos ligando $x$ a $y$. Como $d_{P_{i}}(v) \geq 2$, segue que $d_{G}(v) \geq$ $\sum_{i=1}^{k} d_{P_{i}}(v)=2 k$, uma contradição.

\subsubsection{Alta aresta-conexidade}

Nesta subseção, apresentamos algumas propriedades exibidas por grafos que possuem uma dada aresta-conexidade.

Se $G$ é um grafo que contém $2 k$ árvores geradoras duas-a-duas aresta-disjuntas, então, claramente, $G$ é $2 k$-aresta-conexo. A recíproca não é verdadeira, mas como enunciado no próximo teorema, todo grafo $2 k$-aresta-conexo contém $k$ tais árvores.

Teorema 5.8 (Nash-Williams [NW61]; Tutte [Tut61]). Seja $k$ um inteiro positivo. Se G é um grafo $2 k$-aresta-conexo, então $G$ contém $k$ árvores geradoras duas-a-duas aresta-disjuntas.

O seguinte resultado recente de Lovász, Thomassen, Wu e Zhang [LTWZ13] melhora um resultado de Thomassen [Tho12], e nos permite, sob condições necessárias óbvias, e uma arestaconexidade mínima, encontrar orientações especiais das arestas de um grafo.

Teorema 5.9 (Lovász-Thomassen-Wu-Zhang [LTWZ13]). Sejam $k \geq 3$ um inteiro positivo e $G$ um grafo $(3 k-2)$-aresta-conexo. Seja $p: V(G) \rightarrow\{0, \ldots, k-1\}$ tal que $\sum_{v \in V(G)} p(v) \equiv|E(G)|$ $(\bmod k)$. Então existe uma orientação $O$ de $G$ tal que $d_{O}^{+}(v) \equiv p(v)(\bmod k)$, para todo vértice $v$ de $G$.

Combinando os dois teoremas anteriores, provaremos o seguinte lema, que nos permite tratar grafos bipartidos altamente aresta-conexos como grafos regulares. Este lema é uma generalização simples da Proposição 2 em [Tho13a].

Lema 5.10. Sejam $k \geq 3$ e $r$ inteiros positivos. Se $G=\left(A_{1}, A_{2} ; E\right)$ é um grafo bipartido ( $6 k+$ $4 r-4)$-aresta-conexo e $|E|$ é divisivel por $k$, então $G$ admite uma decomposição em dois grafos geradores r-aresta-conexos $G_{1}$ e $G_{2}$ tais que, o grau em $G_{i}$ de cada vértice de $A_{i}$ é divisivel por $k$, para $i=1,2$.

Demonstração. Sejam $k, r$ e $G=\left(A_{1}, A_{2}, E\right)$ como no enunciado do lema. Pelo Teorema 5.8, $G$ contém $3 k+2 r-2$ árvores geradoras duas-a-duas aresta-disjuntas. Seja $H_{1}$ a união de $r$ dessas árvores, seja $H_{2}$ a união de outras $r$ dessas árvores, e seja $H_{3}=G-E\left(H_{1}\right)-E\left(H_{2}\right)$. Temos que $H_{1}$ e $H_{2}$ são $r$-aresta-conexos, e $H_{3}$ é $(3 k-2)$-aresta-conexo.

Tome $p: V\left(H_{3}\right) \rightarrow\{0, \ldots, k-1\}$ tal que $p(v) \equiv(k-1) d_{H_{1}}(v)(\bmod k)$ se $v$ é um vértice de $A_{1}$, e $p(v) \equiv(k-1) d_{H_{2}}(v)(\bmod k)$ se $v$ é um vértice de $A_{2}$. Logo, vale o seguinte, onde as congruências 
são tomadas módulo $k$.

$$
\begin{aligned}
\sum_{v \in V(G)} p(v) & =\sum_{v \in A_{1}} p(v)+\sum_{v \in A_{2}} p(v) \\
& \equiv(k-1)\left(\left|E\left(H_{1}\right)\right|+\left|E\left(H_{2}\right)\right|\right) \\
& \equiv(k-1)\left(|E|-\left|E\left(H_{3}\right)\right|\right) \\
& \equiv k\left(|E|-\left|E\left(H_{3}\right)\right|\right)-|E|+\left|E\left(H_{3}\right)\right| \\
& \equiv\left|E\left(H_{3}\right)\right| .
\end{aligned}
$$

Como $H_{3}$ é um subgrafo gerador $(3 k-2)$-aresta-conexo de $G$, pelo Teorema 5.9 existe uma orientação $O$ de $H_{3}$ tal que $d_{O}^{+}(v) \equiv p(v)(\bmod k)$ para todo $v \in V\left(H_{3}\right)=V(G)$. Para $i=1,2$ seja $G_{i}$ o grafo $H_{i}$ juntamente com as arestas de $H_{3}$ que saem de $A_{i}$ na orientação $O$ (note que $\left.E=E\left(G_{1}\right) \cup E\left(G_{2}\right)\right)$. Logo, $d_{G_{i}}(v)=d_{H_{i}}(v)+d_{O}^{+}(v) \equiv k d_{H_{i}}(v) \equiv 0(\bmod k)$ para todo vértice $v$ em $A_{i}$, e além disso, $G_{i}$ é $r$-aresta-conexo (porque contém $H_{i}$ ).

O teorema seguinte é uma generalização de um resultado de Petersen [Pet91] que diz que todo grafo cúbico sem pontes contém um emparelhamento perfeito.

Teorema 5.11 (Von Baebler [VB37] (see also [AK11, Theorem 2.37])). Sejam $r \geq 2$ um inteiro positivo, e $G$ um multigrafo $r$-regular e $(r-1)$-aresta-conexo com um número par de vértices. Então $G$ contém um 1-fator.

Os próximos resultados são obtidos pela generalização de uma técnica usada por Bárat e Gerbner [BG14], e serão usados na prova do Lema 5.14.

Teorema 5.12 (Teorema $20 \mathrm{em}$ [ENV02]). Seja $m$ um inteiro positivo. Se G é um grafo $m$-arestaconexo, então $G$ contém uma árvore geradora $T$ tal que $d_{T}(v) \leq\left\lceil d_{G}(v) / m\right\rceil+2$ para todo vértice $v$ de $G$.

Corolário 5.13. Seja $m$ um inteiro positivo. Se $G$ é um grafo $m$-aresta-conexo, então $G$ contém uma árvore geradora $T$ tal que $d_{T}(v) \leq 4 d_{G}(v) / m$ para todo vértice $v$ de $G$.

Demonstração. Da aresta-conexidade de $G$, temos que $d_{G}(v) \geq m$ para todo vértice $v$ de $G$. Combinando isso com o Teorema 5.12, concluímos que $G$ contém uma árvore geradora $T$ tal que $d_{T}(v) \leq\left\lceil d_{G}(v) / m\right\rceil+2 \leq\left(d_{G}(v) / m\right)+3 \leq 4 d_{G}(v) / m$.

Lema 5.14. Sejam $k, m$ e $r$ inteiros positivos, se seja $G=(A, B ; E)$ um grafo bipartido. Se $G$ é $8 m\lceil(k+r) / k\rceil$-aresta-conexo e, para todo $v \in A, d_{G}(v)$ é divisivel por $k+r$, então $G$ admite uma decomposição em grafos geradores $G_{k}$ e $G_{r}$ tal que $G_{k}$ é $m$-aresta-conexo e, para todo vértice $v \in A$, temos que $d_{G_{k}}(v)=\frac{k}{k+r} d_{G}(v)$ e $d_{G_{r}}(v)=\frac{r}{k+r} d_{G}(v)$.

Demonstração. Sejam $k, m, r$ e $G=(A, B ; E)$ como na hipótese do lema. Como $G$ é $8 m\lceil(k+r) / k\rceil$ aresta-conexo, pelo Teorema 5.8 concluímos que $G$ contém pelo menos $4 m\lceil(k+r) / k\rceil$ árvores geradoras duas-a-duas aresta-disjuntas. Agora, particione o conjunto dessas $4 m\lceil(k+r) / k\rceil$ árvores geradoras em $m$ conjuntos, digamos $\mathcal{T}_{1}, \ldots, \mathcal{T}_{m}$, com $4\lceil(k+r) / k\rceil$ árvores geradoras cada, e tome $G_{i}=\bigcup_{T \in \mathcal{T}_{i}} T$, para $i=1, \ldots, m$. 
Claramente, $G_{i}$ é $4\lceil(k+r) / k\rceil$-aresta-conexo. Pelo Corolário 5.13, $G_{i}$ contém uma árvore geradora $T_{i}$ tal que, para todo $v \in V\left(G_{i}\right)$,

$$
d_{T_{i}}(v) \leq \frac{1}{\lceil(k+r) / k\rceil} d_{G_{i}}(v) \leq\left(\frac{k}{k+r}\right) d_{G_{i}}(v) .
$$

Seja $G^{\prime}=\cup_{i=1}^{m} T_{i}$. Claramente, $G^{\prime}$ é $m$-aresta-conexo. Note que, para todo $v \in V(G)$,

$$
d_{G^{\prime}}(v)=\sum_{i=1}^{m} d_{T_{i}}(v) \leq\left(\frac{k}{k+r}\right) \sum_{i=1}^{m} d_{G_{i}}(v) \leq\left(\frac{k}{k+r}\right) d_{G}(v) .
$$

Seja $G_{k}$ o grafo bipartido obtido de $G^{\prime}$ pela adição, para todo vértice $v$ em $A$, de exatamente $(k /(k+r)) d_{G}(v)-d_{G^{\prime}}(v)$ arestas de $G-E\left(G^{\prime}\right)$ que são incidentes a $v$ (note que $(k /(k+r)) d_{G}(v)$ é um inteiro). Portanto, todo vértice $v \in A$ tem grau exatamente $(k /(k+r)) d_{G}(v)$ em $G_{k}$. Para concluir a prova, tome $G_{r}=G-E\left(G_{k}\right)$.

\subsection{Fatorizações}

O objetivo desta seção é mostrar que alguns grafos bipartidos altamente aresta-conexos admitem decomposições "bem estruturadas", chamadas de bifatorizações, que são estruturas importantes na prova dos teoremas principais deste capítulo (mostrados na Seção 5.4).

\subsubsection{Fatorizações fracionárias}

Antes de definir bifatorizações, precisamos generalizar os conceitos de fatores e de fatorizações. Nós estendemos as ideias desenvolvidas em [BMOWa] e formalizamos algumas ideias apresentadas em [Tho08a].

Definição 5.15 (Fator). Sejam $r$ e $\ell$ inteiros positivos e $G=(V, E)$ um grafo. Sejam $X \subset V$ e $F \subset$ E. Dizemos que $F$ é um $(X, r, \ell)$-fator de $G$ se $d_{F}(v)=(r / \ell) d_{G}(v)$, para todo $v \in X$.

Note que se $G$ é um grafo $\ell$-regular, então um $(V(G), r, \ell)$-fator é precisamente um $r$-fator de $G$.

Definição 5.16 (Fatorização fracionária). Sejam $\ell$ e $k$ inteiros positivos tais que $\ell-k$ é um número par positivo. Seja $G=(V, E)$ um grafo e seja $X \subset V$. Dizemos que uma partição $\mathcal{F}=$ $\left\{M_{1}, \ldots, M_{k}, F_{1}, \ldots, F_{(\ell-k) / 2}\right\}$ de $E$ é uma $(X, k, \ell)$-fatorização fracionária de $G$ se tem as seguintes propriedades.

- $M_{i}$ é um $(X, 1, \ell)$-fator de $G$, para $1 \leq i \leq k$; e

- $F_{j}$ é um $(X, 2, \ell)$-fator Euleriano de $G$, para $1 \leq j \leq(\ell-k) / 2$.

Note que, se $G$ contém um $(X, 1, \ell)$-fator, então $d_{G}(v)$ é divisível por $\ell$ para todo $v \in X$. Portanto, esse fato implica que, se $G$ admite uma $(X, k, \ell)$-fatorização fracionária, então $d(v)$ é divisível por $\ell$ para todo $v \in X$. O próximo lema é o núcleo desta seção.

Lema 5.17. Seja $\ell$ um inteiro positivo impar. $S e G=(A, B ; E)$ é um grafo bipartido $(\ell-1)$-arestaconexo tal que $d_{G}(v)$ é divisivel por $\ell$ para todo $v \in A$, então $G$ admite uma $(A, 1, \ell)$-fatorização fracionária. 
Demonstração. Sejam $\ell$ e $G=(A, B ; E)$ como na hipótese. Primeiramente, queremos aplicar o Lema 5.5 para obter um grafo $(\ell-1)$-aresta-conexo $G^{\prime}$ com grau máximo $2 \ell-3$. Para fazer isso, para todo vértice $v \in B$, tomamos inteiros $s_{v} \geq 1$ e $0 \leq r_{v}<\ell-1$ tais que $d_{G}(v)=(\ell-1) s_{v}+r_{v}$. Colocamos $d_{1}^{v}=\ell-1+r_{v}$ e $d_{2}^{v}=\cdots=d_{s_{v}}^{v}=\ell-1$. Além disso, para todo vértice $v \in A$, colocamos $s_{v}=d_{G}(v) / \ell$ e $d_{i}^{v}=\ell$ para $1 \leq i \leq s_{v}$. Pelo Lema 5.5 (aplicado com parâmetros $\ell-1$ e os inteiros $s_{v}$, $d_{i}^{v}\left(1 \leq i \leq s_{v}\right)$ para todo $\left.v \in V(G)\right)$, existe um grafo bipartido $(\ell-1)$-aresta-conexo $G^{\prime}$ obtido de $G$ pelo splitting de cada vértice $v$ de $A$ em $s_{v}$ vértices de grau $\ell$, e cada vértice $v$ de $B$ em um vértice de grau $\ell-1+r_{v}<2 \ell-2$ e $s_{v}-1$ vértices de grau $\ell-1$. Sejam $A^{\prime}$ e $B^{\prime}$ os conjuntos de vértices de $G^{\prime}$ obtidos dos vértices de $A$ e $B$, respectivamente. Para facilitar a notação, se $v \in\left(A^{\prime} \cup B^{\prime}\right) \backslash(A \cup B)$ também denotamos por $v$ o vértice em $A \cup B$ que deu origem a $v$.

O próximo passo é obter um multigrafo $\ell$-regular $G^{*}$ a partir de $G^{\prime}$ por operações de lifting. Para isso, adicionamos algumas arestas a $A^{\prime}$ e removemos os vértices de grau par de $B^{\prime}$ com aplicações sucessivas do Teorema de Lifting de Mader da seguinte forma. Seja $G_{0}^{\prime}, G_{1}^{\prime}, \ldots, G_{\lambda}^{\prime}$ uma sequência maximal de grafos tais que $G_{0}^{\prime}=G^{\prime}$ e (para $\left.i \geq 0\right) G_{i+1}^{\prime}$ é o grafo obtido de $G_{i}^{\prime}$ pela aplicação de um lifting admissível num vértice arbitrário $v \in B^{\prime} \operatorname{com} d_{G^{\prime}}(v) \notin\{1,2, \ell\}$.

Lembremos que, dados quaisquer dois vértices distintos de $G^{\prime}$, digamos $x$ e $y$, denotamos por $p_{G^{\prime}}(x, y)$ o maior número de caminhos aresta-disjuntos ligando $x$ e $y$ em $G^{\prime}$. Afirmamos que $p_{G_{i}^{\prime}}(x, y) \geq \ell-1$ para quaisquer $x, y$ em $A^{\prime}$ e todo $i \geq 0$. Claramente, $p_{G_{0}^{\prime}}(x, y) \geq \ell-1$ vale para quaisquer $x, y$ em $A^{\prime}$, uma vez que $G^{\prime}$ é $(\ell-1)$-aresta-conexo. Fixe $i \geq 0$ e suponha que $p_{G_{i}^{\prime}}(x, y) \geq \ell-1$ valha para quaisquer $x, y$ em $A^{\prime}$. Sejam $x, y$ dois vértices em $A^{\prime}$. Como $G_{i+1}^{\prime}$ é o grafo obtido de $G_{i}^{\prime}$ pela aplicação de um lifting admissível num vértice $v$ de $B^{\prime}$, temos que $p_{G_{i+1}^{\prime}}(x, y) \geq p_{G_{i}^{\prime}}(x, y) \geq \ell-1$.

Afirmamos que, se $v \in B^{\prime}$ então $d_{G_{\lambda}^{\prime}}(v) \in\{2, \ell\}$. Suponha, por contradição, que exista um vértice $v$ em $B^{\prime}$ tal que $d_{G_{\lambda}^{\prime}}(v) \notin\{2, \ell\}$. Note que $d_{G_{i}^{\prime}}(u) \geq d_{G_{i+1}^{\prime}}(u) \geq 2$ para todo $u \in V\left(G^{\prime}\right)$ e todo $0 \leq i \leq \lambda$. Como $d_{G^{\prime}}(u) \leq 2 \ell-3$ para todo $u \in V\left(G^{\prime}\right)$, temos que $2 \leq d_{G_{i}^{\prime}}(u) \leq 2 \ell-3$ para todo $0 \leq i \leq \lambda$. Portanto, $2 \leq d_{G_{\lambda}^{\prime}}(v) \leq 2 \ell-3$. Como $d_{G_{\lambda}^{\prime}}(v) \leq 2 \ell-3$, e para quaisquer dois vizinhos $x$ e $y$ de $v$ temos que $p_{G_{\lambda}^{\prime}}(x, y) \geq \ell-1$, pelo Lema 5.7 segue que $v$ não é um vértice-de-corte de $G_{\lambda}^{\prime}$. Assim, pelo Teorema de Lifting de Mader (Teorema 5.6) aplicado a $G_{\lambda}^{\prime}$, existe um lifting admissível em $v$. Portanto, $G_{0}^{\prime}, G_{1}^{\prime}, \ldots, G_{\lambda}^{\prime}$ não é maximal, uma contradição.

Em $G_{\lambda}^{\prime}$ o conjunto $B^{\prime}$ pode conter alguns vértices de grau 2 . Para cada tal vértice $v$, se $u$ e $w$ são os vizinhos de $v$, aplicamos um $u w$-lifting em $v$, e removemos o vértice $v$, i.e, fazemos a supressão de $v$. Seja $G^{*}$ o grafo obtido pela aplicação desse processo em todos os vértices de grau 2 em $B^{\prime}$. Note que o número de caminhos aresta-disjuntos ligando dois vértices distintos de $A^{\prime}$ não é reduzido, i.e, $p_{G^{*}}(x, y) \geq p_{G_{\lambda}}(x, y) \geq \ell-1$ para todo $x, y$ em $A^{\prime}$. Claramente, o conjunto de vértices de $G^{*}$ que pertence a $B^{\prime}$ é um conjunto independente; nós o denotamos por $B^{*}$ (eventualmente, $B^{*}=\emptyset$ ). Além disso, todo vértice em $B^{*}$ tem grau $\ell$.

Afirmação 5.18. $G^{*} e ́(\ell-1)$-aresta-conexo.

Demonstração. Seja $Y \subset V\left(G^{*}\right)$. Suponha que exista pelo menos um vértice $x$ de $A^{\prime}$ em $Y$ e pelo menos um vértice $y$ de $A^{\prime}$ em $V\left(G^{*}\right)-Y$. Como existem pelo menos $\ell-1$ caminhos aresta-disjuntos ligando $x$ a $y$, existem pelo menos $\ell-1$ arestas com vértices em ambos $Y$ e $V\left(G^{*}\right)-Y$. Agora, suponha que $A^{\prime} \subset Y$ (caso contrário, $A^{\prime} \subset V\left(G^{*}\right)-Y$, e tomamos $V\left(G^{*}\right)-Y$ no lugar de $Y$ ), e então $V\left(G^{*}\right)-Y \subset B^{*}$. Como $B^{*}$ é um conjunto independente, todas as arestas com um vértice 
em $V\left(G^{*}\right)-Y$ devem ter o outro vértice em $A^{\prime}$. Como todo vértice em $B^{*}$ tem grau $\ell$, existem pelo menos $\ell$ arestas com vértices em ambos $Y$ e $V\left(G^{*}\right)-Y$.

Concluímos que $G^{*}$ é um multigrafo $\ell$-regular $(\ell-1)$-aresta-conexo com conjunto de vértices $A^{\prime} \cup B^{*}$, onde $B^{*}$ é um conjunto independente.

Como todo vértice de $G^{*}$ tem grau ímpar, $\left|V\left(G^{*}\right)\right|$ é par. Pelo Teorema 5.11, $G^{*}$ contém um emparelhamento perfeito $M^{*}$. Como o multigrafo $J^{*}=G^{*}-M^{*}$ é $(\ell-1)$-regular, o Teorema 2.1 implica que $J^{*}$ admite uma decomposição em 2 -fatores, digamos $F_{1}^{*}, \ldots, F_{(\ell-1) / 2}^{*}$. Portanto, $M^{*}, F_{1}^{*}, \ldots, F_{(\ell-1) / 2}^{*}$ é uma partição de $E\left(G^{*}\right)$.

Agora, vamos voltar ao grafo bipartido $G$. Seja $x y$ uma aresta de $G^{*}$. Se $x \in A^{\prime}$ e $y \in B^{*}$, então $x y$ corresponde a uma aresta de $G$. Por outro lado, se $x, y \in A^{\prime}$, então existe um vértice $v_{x y}$ de $B^{\prime}$ e duas arestas $x v_{x y}$ e $v_{x y} y$ em $E\left(G^{\prime}\right)$. Além disso, $x y$ foi obtida por um $x y$-lifting em $v_{x y}$ (ou pela aplicação do Teorema de Lifting de Mader, ou pela supressão de vértices de grau 2). Então, cada aresta de $G^{*}$ representa uma aresta de $G$ ou um 2-caminho em $G$ tal que os vértices internos desses 2-caminhos estão sempre em $B$. Para cada aresta $x y \in E\left(G^{*}\right)$, defina $f(x y)=\{x y\}$ se $x \in A^{\prime}$ e $y \in B^{*}$, e $f(x y)=\left\{x v_{x y}, v_{x y} y\right\}$ se $x, y \in A^{\prime}$. Note que $f(x y) \subset E(G)$ para cada aresta $x y \in E\left(G^{*}\right)$. Para um conjunto $S \subset E\left(G^{*}\right)$, coloque $f(S)=\cup_{e \in S} f(e)$. A partição de $E\left(G^{*}\right)$ em $M^{*}, F_{1}^{*}, \ldots, F_{(\ell-1) / 2}^{*}$ induz uma partição de $E(G)$ em $M=f\left(M^{*}\right)$ e $F_{i}=f\left(F_{i}^{*}\right)$ para $1 \leq i \leq(\ell-1) / 2$.

Vamos provar que $\left\{M, F_{1}, \ldots, F_{(\ell-1) / 2}\right\}$ é uma $(A, 1, \ell)$-fatorização fracionária. Fixe um índice $i \in\{1, \ldots,(\ell-1) / 2\}$. Vamos mostrar que $M$ é um $(A, 1, \ell)$-fator de $G$ e $F_{i}$ é um $(A, 2, \ell)$-fator Euleriano de $G$. Seja $v$ um vértice de $A$ em $G$ e coloque $d^{\prime}(v)=d(v) / \ell$. Então sabemos que $v$ é representado por $d^{\prime}(v)$ vértices em $G^{*}$. Como $M^{*}$ é um emparelhamento perfeito em $G^{*}$, existem $d^{\prime}(v)$ arestas de $M$ entrando em $v$ e, como $F_{i}^{*}$ é um 2-fator em $G^{*}$, existem $2 d^{\prime}(v)$ arestas de $F_{i}$ incidentes a $v$. Finalmente, como $F_{i}^{*}$ é Euleriano, o conjunto $F_{i}$ é Euleriano, concluindo a prova.

Corolário 5.19. Seja $\ell$ um inteiro positivo par. Se $G=(A, B ; E)$ é um grafo bipartido $16(\ell-2)$ aresta-conexo tal que $d_{G}(v)$ é divisivel por $\ell$ para todo $v \in A$, então $G$ admite uma $(A, 2, \ell)$ fatorização fracionária.

Demonstração. Sejam $\ell$ e $G=(A, B ; E)$ como na hipótese. Afirmamos que $G$ contém um $(A, 1, \ell)$ fator $F$ tal que $G-F$ é $\left(\ell-2\right.$ )-aresta-conexo (note que $d_{G-F}(v)$ é divisível por $\ell-1$ para todo $v \in A$ ). Como $G$ é $16(\ell-2)=8(\ell-2)\lceil\ell /(\ell-1)\rceil$-aresta-conexo, pelo Lema 5.14 (aplicado com parâmetros $k=\ell-1, m=\ell-2$ e $r=1$ ), o grafo $G$ admite uma decomposição em grafos $G_{k}$ e $G_{r}$ tal que $G_{k}$ é $(\ell-2)$-aresta-conexo e $d_{G_{k}}(v)=((\ell-1) / \ell) d_{G}(v)$, e $d_{G_{r}}(v)=(1 / \ell) d_{G}(v)$ para todo $v \in A$. Portanto, $E\left(G_{r}\right)$ é um $(A, 1, \ell)$-fator. Pelo Lema 5.17, $G_{k}$ admite uma $(A, 1, \ell-1)$ fatorização fracionária $\mathcal{F}$. Portanto, como $d_{G_{k}}(v)=((\ell-1) / \ell) d_{G}(v)$ para todo $v \in A$, concluímos que $\mathcal{F} \cup\left\{E\left(G_{r}\right)\right\}$ é uma $(A, 2, \ell)$-fatorização fracionária de $G$.

\subsubsection{Bifatorizações}

Para obter uma decomposição de grafos bipartidos altamente aresta-conexos $G$ em caminhos de comprimento fixo $\ell$, precisamos combinar fatorizações fracionárias. Mais especificamente, decompomos $G$ em grafos $G_{1}$ e $G_{2}$ e então combinamos uma fatorização fracionária de $G_{1}$ com uma fatorização fracionária de $G_{2}$. Esse processo, chamado de bifatorização, é definido a seguir. 
Definição 5.20 (Bifatorização). Sejam $\ell$ e $k$ inteiros positivos tais que $\ell-k$ é um número par positivo, e seja $G=\left(A_{1}, A_{2} ; E\right)$ um grafo bipartido. Sejam $\mathcal{F}_{1}, \mathcal{F}_{2}$ famílias de subconjuntos de $E$ e $G_{i}=G\left[\cup_{F \in \mathcal{F}_{i}} F\right]$, para $i=1,2$. Dizemos que $\mathbb{F}=\left(\mathcal{F}_{1}, \mathcal{F}_{2}\right)$ é uma $(k, \ell)$-bifatorização de $G$ se tem as seguintes propriedades.

(i) $\left\{G_{1}, G_{2}\right\}$ é uma decomposição de $G$; e

(ii) $\mathcal{F}_{i}$ é uma $\left(A_{i}, k, \ell\right)$-fatorização fracionária de $G_{i}$, para $1 \leq i \leq 2$.

Se $G$ admite uma $(k, \ell)$-bifatorização, dizemos que $G$ é $(k, \ell)$-bifatorável.

O próximo conceito será usado para garantir que $G_{1}$ e $G_{2}$ tenham graus mínimos suficientemente altos.

Definição 5.21 (Bifatorização forte). Seja $\ell$ um inteiro positivo par. Seja $G=\left(A_{1}, A_{2} ; E\right)$ um grafo bipartido que admite uma $(2, \ell)$-bifatorização $\mathbb{F}=\left(\mathcal{F}_{1}, \mathcal{F}_{2}\right)$. Seja $E_{i}=\bigcup_{F \in \mathcal{F}_{i}} F$ para $1 \leq i \leq 2$. Dizemos que $\mathbb{F}$ é forte se $d_{E_{i}}(v) \geq(\ell / 2)(\ell / 2+1)$ para todo $v$ em $A_{i}$ para $1 \leq i \leq 2$. Se $G$ admite uma $(2, \ell)$-bifatorização forte, dizemos que $G$ é fortemente $(2, \ell)$-bifatorável.

Para facilitar a notação, se $F$ pertence a $\mathcal{F}_{1}$ ou $\mathcal{F}_{2}$, então dizemos que $F$ é um elemento de $\mathbb{F}$. No que se segue, damos condições suficientes para um grafo bipartido ser fortemente bifatorável.

Lema 5.22. Seja $\ell$ um inteiro positivo par. Seja $r=\max \{16(\ell-2),(\ell / 2)(\ell / 2+1)\}$. Se $G$ é um grafo bipartido $(6 \ell+4 r-4)$-aresta-conexo tal que $|E(G)|$ é divisivel por $\ell$, então $G$ é fortemente $(2, \ell)$-bifatorável.

Demonstração. Sejam $\ell, r$ e $G=(A, B ; E)$ como na hipótese. Pelo Lema 5.10 (aplicado com $\ell$ e $r$ ), o grafo $G$ pode ser decomposto em dois grafos geradores aresta-disjuntos e $r$-aresta-conexos $G_{1}$ e $G_{2}$ tais que todos os vértices de $A$ têm grau divisível por $\ell$ em $G_{1}$, e todos os vértices de $B$ têm grau divisível por $\ell$ em $G_{2}$. Mas como $r \geq 16(\ell-2)$, pelo Corolário 5.19 (aplicado com $\ell$ ), concluímos que $G_{1}$ admite uma $(A, 2, \ell)$-fatorização fracionária e $G_{2}$ admite uma $(B, 2, \ell)$-fatorização fracionária. Portanto, $G$ é $(2, \ell)$-bifatorável. Como $G_{1}$ e $G_{2}$ são $r$-aresta-conexos, temos que $d_{G_{1}}(v) \geq$ $r \geq(\ell / 2)(\ell / 2+1)$ para todo $v \in A$, e $d_{G_{2}}(v) \geq r \geq(\ell / 2)(\ell / 2+1)$ para todo $v \in B$, de onde concluímos que $G$ é fortemente $(2, \ell)$-bifatorável.

O resultado que será usado mais adiante é, especificamente, o seguinte corolário.

Corolário 5.23. Seja $\ell$ um inteiro positivo e seja $r=\max \{32(\ell-1), \ell(\ell+1)\}$. Se $G$ é um grafo bipartido $(12 \ell+4 r-4)$-aresta-conexo tal que $|E(G)|$ é divisivel por $2 \ell$, então $G$ é fortemente $(2,2 \ell)$ bifatorável.

\subsection{Decomposições de grafos bifatoráveis em $\ell$-caminhos}

Nesta seção provamos que grafos bipartidos que admitem bifatorizações fortes podem ser decompostos em caminhos de comprimento fixo. Aqui, não fazemos o uso de alta aresta-conexidade. Primeiramente, adaptamos o conceito de balanceamento de uma rastro-decomposição para lidar com tais grafos. As proposições 5.25 e 5.26 lidam com os casos bases da nossa indução. 
Definição 5.24 (Rastro-decomposições balanceadas). Seja $\ell$ um inteiro positivo. Seja $G=(A, B ; E)$ um grafo bipartido que admite uma $(2,2 \ell)$-bifatorização $\mathbb{F}=\left(\mathcal{F}_{1}, \mathcal{F}_{2}\right)$, e seja $G_{i}=G\left[\bigcup_{F \in \mathcal{F}_{i}} F\right]$ para $i=1,2$. Sejam $M_{1}, N_{1}$ os $(A, 1,2 \ell)$-fatores de $\mathbb{F}$, e sejam $M_{2}, N_{2}$ os $(B, 1,2 \ell)$-fatores de $\mathbb{F}$. Dizemos que uma $\ell$-decomposição $\mathcal{B}$ de $G$ é $\mathbb{F}$-balanceada se tem as seguintes propriedades.

- $\mathcal{B}(v)=d_{G_{1}}(v) / \ell+d_{M_{2}}(v)+d_{N_{2}}(v)$, para todo $v \in A ; e$

- $\mathcal{B}(v)=d_{G_{2}}(v) / \ell+d_{M_{1}}(v)+d_{N_{1}}(v)$, para todo $v \in B$.

Nosso objetivo é provar o Teorema 5.27, que garante que é possível obter uma $\ell$-decomposição em caminhos $\mathbb{F}$-balanceada e $\ell$-completa a partir de uma $(2,2 \ell)$-bifatorização forte $\mathbb{F}$. Primeiramente, mostramos que a partir de uma $(2,4)$-bifatorização podemos obter uma 2-decomposição em caminhos balanceada.

Proposição 5.25. Se $G$ é um grafo bipartido que admite uma $(2,4)$-bifatorização $\mathbb{F}$, então $G$ admite uma 2-decomposição em caminhos $\mathbb{F}$-balanceada.

Demonstração. Seja $G=(A, B ; E)$ um grafo bipartido que admite uma $(2,4)$-bifatorização $\mathbb{F}=$ $\left(\mathcal{F}_{1}, \mathcal{F}_{2}\right)$, onde $\mathcal{F}_{i}=\left\{M_{i}, N_{i}, F_{i}\right\}$ para $i=1,2$. Seja $O_{F_{i}}$ uma orientação Euleriana de $G\left[F_{i}\right]$, para $i=1,2$. Seja $\mathcal{C}_{1}$ o conjunto de componentes de $G\left[F_{1}\right]$. Seja $T$ um elemento de $\mathcal{C}_{1}$ e $B_{T}=$ $a_{0} b_{0} a_{1} b_{1} \cdots a_{s} b_{s} a_{0}$ um rastro de $T$, onde $a_{i} \in A$, e $b_{i} \in B$, para $1 \leq i \leq s$. Temos que $\mathcal{B}_{T}^{\prime}=$ $\left\{a_{i} b_{i} a_{i+1}: 0 \leq i \leq s\right\}$, onde $a_{s+1}=a_{0}$, é uma 2-decomposição de $T$ na qual todos rastros têm seus vértices finais e iniciais em $A$. Portanto, $\mathcal{B}_{1}^{\prime}=\cup_{T \in \mathcal{C}_{1}} \mathcal{B}_{T}^{\prime}$ é uma 2-decomposição de $G\left[F_{1}\right]$ na qual todos os rastros têm seus vértices finais e iniciais em $A$. Analogamente, $G\left[F_{2}\right]$ admite uma 2-decomposição $\mathcal{B}_{2}^{\prime}$ na qual todos os rastros têm seus vértices finais e iniciais em $B$.

Seja $v$ um vértice de $A$. Como $M_{1}$ e $N_{1}$ são $(A, 1,4)$-fatores de $G$, temos que $d_{M_{1}}(v)=d_{N_{1}}(v)$. Logo, o número de arestas em $M_{1} \cup N_{1}$ incidentes a $v$ é par, e podemos decompor as arestas em $M_{1} \cup N_{1}$ incidentes a $v$ em caminhos de comprimento 2 tais que cada caminho tem seus vértices finais e iniciais em $B$. Tomando quaisquer rastros desses caminhos, obtemos uma 2-decomposição $\mathcal{B}_{1}^{\prime \prime}$ das arestas em $M_{1} \cup N_{1}$ tal que cada caminho tem seus vértices finais e iniciais em $B$. Analogamente, há uma 2-decomposição $\mathcal{B}_{2}^{\prime \prime}$ das arestas em $M_{2} \cup N_{2}$ tal que cada rastro tem seus vértices finais e iniciais em $A$.

Seja $\mathcal{B}=\mathcal{B}_{1}^{\prime} \cup \mathcal{B}_{2}^{\prime} \cup \mathcal{B}_{1}^{\prime \prime} \cup \mathcal{B}_{2}^{\prime \prime}$. Note que somente os rastros em $\mathcal{B}_{1}^{\prime}$ e em $\mathcal{B}_{2}^{\prime \prime}$ têm vértices finais e iniciais em $A$, e analogamente somente os caminhos em $\mathcal{B}_{2}^{\prime}$ e em $\mathcal{B}_{1}^{\prime \prime}$ têm vértices finais e iniciais em $B$. Portanto, se $v$ é um vértice de $A$, então $\mathcal{B}(v)=\mathcal{B}_{1}^{\prime}(v)+\mathcal{B}_{2}^{\prime \prime}(v)=d_{G}(v) / 2+d_{M_{2}}(v)+d_{N_{2}}(v)$, e se $v$ é um vértice de $B$, então $\mathcal{B}(v)=\mathcal{B}_{2}^{\prime}(v)+\mathcal{B}_{2}^{\prime \prime}(v)=d_{G}(v) / 2+d_{M_{1}}(v)+d_{N_{1}}(v)$. Logo, $\mathcal{B}$ é uma 2-decomposição em caminhos $\mathbb{F}$-balanceada de $G$.

O resultado a seguir se assemelha à parte da prova dada por Thomassen [Tho08a] para decomposição de grafos altamente aresta-conexos em caminhos de comprimento 3, porém aqui precisamos garantir que a decomposição obtida é balanceada.

Proposição 5.26. Se $G$ é um grafo bipartido que admite uma $(2,6)$-bifatorização $\mathbb{F}$, então $G$ admite uma 3-decomposição em caminhos $\mathbb{F}$-balanceada.

Demonstração. Seja $G=(A, B ; E)$ um grafo bipartido que admite uma $(2,6)$-bifatorização $\mathbb{F}=$ $\left(\mathcal{F}_{1}, \mathcal{F}_{2}\right)$, onde $\mathcal{F}_{i}=\left\{M_{i}, N_{i}, F_{i}, H_{i}\right\}$ para $i=1,2$. Seja $\mathcal{C}_{1}$ o conjunto das componentes de $G\left[F_{1} \cup H_{1}\right]$. 
Seja $T$ um elemento de $\mathcal{C}_{1}$ e $B_{T}=a_{0} b_{0} a_{1} b_{1} \cdots a_{s} b_{s} a_{0}$ um rastro de $T$, onde $a_{i} \in A$ e $b_{i} \in B$, para $1 \leq i \leq s$. Temos que $\mathcal{B}_{T}^{\prime}=\left\{a_{i} b_{i} a_{i+1}: 0 \leq i \leq s\right\}$, tomando $a_{s+1}=a_{0}$, é uma 2-decomposição de $T$ na qual todos os rastros têm seus vértices finais e iniciais em $A$. Portanto, $\mathcal{B}_{1}^{\prime}=\cup_{T \in \mathcal{C}_{1}} \mathcal{B}_{T}^{\prime}$ é uma 2-decomposição de $G\left[F_{1} \cup H_{1}\right]$ na qual todos os rastros têm seus vértices finais e iniciais em $A$. Analogamente, $G\left[F_{2} \cup H_{2}\right]$ admite uma 2-decomposição $\mathcal{B}_{2}^{\prime}$ na qual todos os rastros têm seus vértices finais e iniciais em $B$.

Seja $G_{i}=G\left[M_{i} \cup N_{i} \cup F_{i} \cup H_{i}\right]$ para $i=1,2$. Note que, como $M_{1} \cup N_{1}$ é um $(A, 2,6)$-fator e $F_{1} \cup H_{1}$ é um $(A, 4,6)$-fator de $G_{1}$, temos que $d_{F_{1} \cup H_{1}}(v)=(4 / 6) d_{G_{1}}(v)=2 d_{M_{1} \cup N_{1}}(v)$, para todo vértice $v$ em $A$. Note também que o número de rastros em $\mathcal{B}_{1}^{\prime}$ que terminam em um vértice $v$ (note que não estamos contando os rastros que começam em vértices de $A$ ) é igual a $\frac{1}{2} d_{F_{1} \cup H_{1}}(v)=d_{M_{1} \cup N_{1}}(v)$. Logo, podemos estender cada rastro $B$ de $\mathcal{B}_{1}^{\prime}$ com a adição de uma aresta de $M_{1} \cup N_{1}$ no vértice final de $B$, obtendo uma 3 -decomposição em caminhos $\mathcal{B}_{1}$ de $G_{1}$. Analogamente, podemos estender cada rastro $T$ de $\mathcal{B}_{2}^{\prime}$ com a adição de uma aresta de $M_{2} \cup N_{2}$ no vértice final de $T$, obtendo uma 3-decomposição em caminhos $\mathcal{B}_{2}$ de $G_{2}$.

Tome $\mathcal{B}=\mathcal{B}_{1} \cup \mathcal{B}_{2}$. Se $v$ é um vértice de $A$, então o número de rastros tendo $v$ como vértice final ou incial é exatamente $d_{F_{1} \cup H_{1}}(v) / 2+d_{M_{2} \cup N_{2}}(v)$. Portanto, temos que $\mathcal{B}(v)=d_{F_{1}}(v) / 2+$ $d_{H_{1}}(v) / 2+d_{M_{2}}(v)+d_{N_{2}}(v)=d_{G_{1}}(v) / 3+d_{M_{2}}(v)+d_{N_{2}}(v)$. Analogamente, temos que $\mathcal{B}(v)=$ $d_{G_{2}}(v) / 3+d_{M_{1}}(v)+d_{N_{1}}(v)$ para todo vértice $v$ em $B$. Logo, $\mathcal{B}$ é uma 3-decomposição em caminhos $\mathbb{F}$-balanceada de $G$.

Agora estamos prontos para provar o resultado principal desta seção.

Teorema 5.27. Seja $\ell$ um inteiro positivo. Se $G$ é um grafo bipartido que admite uma $(2,2 \ell)$ bifatorização forte $\mathbb{F}$, então $G$ admite uma $\ell$-decomposição em caminhos $\mathbb{F}$-balanceada.

Demonstração. A prova segue por indução em $\ell$. Pela Proposição 5.25, o enunciado é verdadeiro para $\ell=2$; e pela Proposição 5.26, o enunciado é verdadeiro para $\ell=3$. Suponha que $\ell \geq 4$. Seja $G=$ $\left(A_{1}, A_{2} ; E\right)$ um grafo bipartido com uma $(2,2 \ell)$-bifatorização forte $\mathbb{F}=\left(\mathcal{F}_{1}, \mathcal{F}_{2}\right)$. Afirmamos que $G$ admite uma $\ell$-decomposição $\mathbb{F}$-balanceada e $\ell$-pré-completa. Seja $\mathcal{F}_{1}=\left\{M_{1}, N_{1}, F_{1,1}, \ldots, F_{1, \ell-1}\right\}$ e $\mathcal{F}_{2}=\left\{M_{2}, N_{2}, F_{2,1}, \ldots, F_{2, \ell-1}\right\}$, e seja $G_{i}=G\left[\bigcup_{F \in \mathcal{F}_{i}} F\right]$ para $i=1,2$.

Daqui para frente, fixe $i \in\{1,2\}$. Defina $d^{*}(v)=d_{G_{i}}(v) /(2 \ell)$ para todo vértice $v \in A_{i}$. Note que $d_{F_{i, j}}(v)=2 d^{*}(v)=2 d_{M_{i}}(v)=2 d_{N_{i}}(v)$ para todo vértice $v$ em $A_{i}$ e $1 \leq j \leq \ell-1$. Para $j \in\{\ell-2, \ell-1\}$, seja $O_{F_{i, j}}$ uma orientação Euleriana de $G\left[F_{i, j}\right]$. Seja $F_{i, j}=F_{i, j}^{\text {forw }} \cup F_{i, j}^{\text {back }}$, onde $F_{i, j}^{\text {forw }}$

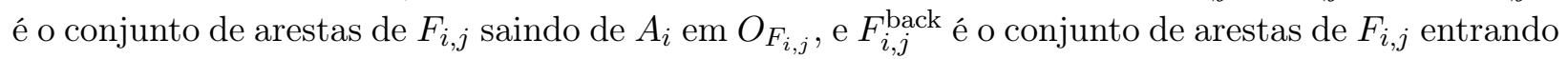
em $A_{i}$ em $O_{F_{i, j}}$. Seja $G^{\prime}=G-M_{1}-N_{1}-M_{2}-N_{2}-F_{1, \ell-2}^{\text {forw }}-F_{1, \ell-1}^{\text {forw }}-F_{2, \ell-2}^{\text {forw }}-F_{2, \ell-1}^{\text {forw }}$, e seja $\mathcal{F}_{i}^{\prime}=$ $\left\{F_{i, \ell-2}^{\text {back }}, F_{i, \ell-1}^{\text {back }}, F_{i, 1}, \ldots, F_{i, \ell-3}\right\}$. Seja $G_{i}^{\prime}=G\left[\bigcup_{F \in \mathcal{F}_{i}^{\prime}} F\right]$. Note que $G_{i}^{\prime}=G_{i}-M_{i}-N_{i}-F_{i, \ell-2}^{\text {forw }}-F_{i, \ell-1}^{\text {forw }}$. Então, para todo $v \in A_{i}$, temos que

$$
d_{G_{i}^{\prime}}(v)=d_{G_{i}}(v)-4 d^{*}(v)=2 \ell d^{*}(v)-4 d^{*}(v)=2(\ell-2) d^{*}(v) .
$$

Afirmação 5.28. $\mathbb{F}^{\prime}=\left(\mathcal{F}_{1}^{\prime}, \mathcal{F}_{2}^{\prime}\right)$ é uma $(2,2(\ell-2))$-bifatorização forte de $G^{\prime}$.

Demonstração. Para provar essa afirmação, devemos provar o seguinte.

(i) $F_{i, \ell-2}^{\text {back }}$ e $F_{i, \ell-1}^{\text {back }}$ são $\left(A_{i}, 1,2(\ell-2)\right)$-fatores de $G_{i}^{\prime}$;

(ii) $F_{i, j}$ é um $\left(A_{i}, 2,2(\ell-2)\right)$-fator Euleriano de $G_{i}^{\prime}$ para $j=1, \ldots, \ell-3$; 
(iii) $d_{G_{i}^{\prime}}(v) \geq(\ell-2)(\ell-1)$ para todo vértice $v \in A_{i}$.

Para provar os itens (i) e (ii), primeiramente note que, para todo $v \in A_{i}$, temos que $d_{F_{i, \ell-2}^{\text {back }}}(v)=$ $d_{F_{i, \ell-1}^{\text {back }}}(v)=d^{*}(v)$ e $d_{F_{i, j}}(v)=2 d^{*}(v)$ para todo $1 \leq j \leq \ell-3$. Por (5.1), concluímos que $F_{i, \ell-2}^{\text {back }}$ e $F_{i, \ell-1}^{\text {back }}$ são $\left(A_{i}, 1,2(\ell-2)\right)$-fatores de $G_{i}^{\prime}$, e $F_{i, j}$ é um $\left(A_{i}, 2,2(\ell-2)\right)$-fator de $G_{i}^{\prime}$. Como $\mathbb{F}$ é uma $(2,2 \ell)$-bifatorização, $F_{1, j}$ e $F_{2, j}$ são grafos Eulerianos para $1 \leq j \leq \ell-3$.

Resta-nos provar o item (iii). Como $d_{G_{i}^{\prime}}(v)=2(\ell-2) d^{*}(v)$ e $d^{*}(v)=d_{G_{i}}(v) /(2 \ell)$ para todo vértice $v \in A_{i}$, temos que $d_{G_{i}^{\prime}}(v)=\frac{2(\ell-2)}{2 \ell} d_{G_{1}}(v)$ para todo $v \in A_{i}$. Como $\mathbb{F}$ é uma $(2,2 \ell)$-bifatorização forte, temos que $d_{G_{i}}(v) \geq \ell(\ell+1)$ para todo $v \in A_{i}$. Logo, $d_{G_{i}^{\prime}}(v) \geq(\ell-2)(\ell+1)>(\ell-2)(\ell-1)$ para todo $v \in A_{i}$.

Como $\mathbb{F}^{\prime}$ é uma $(2,2(\ell-2))$-bifatorização forte de $G^{\prime}$, pela hipótese de indução, $G^{\prime}$ admite uma $(\ell-2)$-decomposição em caminhos $\mathbb{F}^{\prime}$-balanceada $\mathcal{B}^{\prime}$. Como $\mathcal{B}^{\prime}$ é uma $(\ell-2)$-decomposição em caminhos $\mathbb{F}^{\prime}$-balanceada, temos que

- $\mathcal{B}^{\prime}(v)=d_{G_{1}^{\prime}}(v) /(\ell-2)+d_{F_{2, \ell-2}^{\text {back }}}(v)+d_{F_{2, \ell-1}^{\text {back }}}(v)$ para todo $v \in A_{1}$

- $\mathcal{B}^{\prime}(v)=d_{G_{2}^{\prime}}(v) /(\ell-2)+d_{F_{1, \ell-2}^{\text {back }}}(v)+d_{F_{1, \ell-1}^{\text {back }}}(v)$ para todo $v \in A_{2}$.

Agora queremos estender cada $(\ell-2)$-rastro de $\mathcal{B}^{\prime}$ para obter uma $\ell$-decomposição de $G$. Para isso, vamos adicionar arestas de $E(G)-E\left(G^{\prime}\right)$ nos vértices finais dos rastros em $\mathcal{B}^{\prime}$. Para cada vértice $v \in A_{1}\left(v \in A_{2}\right)$, seja $S_{v}$ o conjunto de arestas de $M_{1} \cup N_{1} \cup F_{2, \ell-2}^{\text {forw }} \cup F_{2, \ell-1}^{\text {forw }}\left(M_{2} \cup N_{2} \cup F_{1, \ell-2}^{\text {forw }} \cup F_{1, \ell-1}^{\text {forw }}\right)$ incidentes a $v$. Note que para cada aresta $e$ em $E(G)-E\left(G^{\prime}\right)$ existe exatamente um vértice $v \in V(G)$ tal que $e \in S_{v}$. Então, $\bigcup_{v \in V(G)} S_{v}=E(G)-E\left(G^{\prime}\right)$. Portanto, se provarmos que $\mathcal{B}^{\prime}(v)=\left|S_{v}\right|$ para todo vértice $v \in V(G)$, então podemos estender todo rastro $B$ de $\mathcal{B}^{\prime}$ com a adição de uma aresta em cada um dos vértices finais de $B$.

Afirmação 5.29. $\mathcal{B}^{\prime}(v)=\left|S_{v}\right|$, para todo $v \in V(G)$.

Demonstração. Primeiramente, note que, como $F_{2, \ell-2}$ e $F_{2, \ell-1}$ são Eulerianos, temos que $d_{F_{2, \ell-2}^{\text {back }}}(v)=$ $d_{F_{2, \ell-2}^{\text {forw }}}(v)$ e $d_{F_{2, \ell-1}^{\text {back }}}(v)=d_{F_{2, \ell-1}^{\text {forw }}}(v)$ para todo vértice $v$ em $A_{1}$, e como $F_{1, \ell-2}$ e $F_{1, \ell-1}$ são Eulerianos, temos que $d_{F_{1, \ell-2}^{\text {back }}}(v)=d_{F_{1, \ell-2}^{\text {forw }}}(v)$ e $d_{F_{1, \ell-1}^{\text {back }}}(v)=d_{F_{1, \ell-1}^{\text {forw }}}(v)$ para todo vértice $v$ em $A_{2}$.

Para todo $v \in A_{i}$ e todo $1 \leq j \leq \ell-3$, temos que $d_{G_{i}^{\prime}}(v) /(2(\ell-2))=d_{F_{i, j}}(v) / 2=d^{*}(v)$. Agora, lembremos que para cada vértice $v \in A_{i}$, temos que $d_{M_{i}}(v)=d_{N_{i}}(v)=d^{*}(v)$. Portanto, para todo $v \in A_{1}$, temos que

$$
\begin{aligned}
\mathcal{B}^{\prime}(v) & =d_{G_{1}^{\prime}}(v) /(\ell-2)+d_{F_{2, \ell-2}^{\text {back }}}(v)+d_{F_{2, \ell-1}^{\text {back }}}(v) \\
& =2 d^{*}(v)+d_{F_{2, \ell-2}^{\text {forw }}}(v)+d_{F_{2, \ell-1}^{\text {forw }}}(v) \\
& =d_{M_{1}}(v)+d_{N_{1}}(v)+d_{F_{2, \ell-2}^{\text {forw }}}(v)+d_{F_{2, \ell-1}^{\text {forw }}}(v) \\
& =\left|S_{v}\right| .
\end{aligned}
$$

Similarmente, temos que $\mathcal{B}^{\prime}(v)=\left|S_{v}\right|$ para todo $v \in A_{2}$.

Mostramos que todo rastro $B$ de $\mathcal{B}^{\prime}$ pode ser estendido pela adição de uma aresta em cada um de seus vértices finais. Seja $\mathcal{B}$ a rastro-decomposição obtida com essas extensões. Concluímos que $\mathcal{B}$ é uma $\ell$-decomposição de $G$. 
Afirmação 5.30. $\mathcal{B}$ é $\mathbb{F}$-balanceada.

Demonstração. Seja $x_{0}$ um vértice de $A_{1}$. Primeiramente, vamos mostrar que $\mathcal{B}\left(x_{0}\right) \leq d_{F_{1, \ell-2}^{\text {forw }}}\left(x_{0}\right)+$ $d_{F_{1, \ell-1}^{\text {forw }}}\left(x_{0}\right)+d_{M_{2}}\left(x_{0}\right)+d_{N_{2}}\left(x_{0}\right)$. Se não existe rastro $T=x_{0} x_{1} \cdots x_{\ell}$ em $\mathcal{B}$, onde $x_{0} x_{1}$ é uma aresta de $E(G)-E\left(G^{\prime}\right)$, então $\mathcal{B}\left(x_{0}\right)=0$. Para cada tal rastro $T$, pela construção de $\mathcal{B}$, sabemos que $x_{0} x_{1}$ é um elemento de $S_{x_{1}}$. Como $x_{1}$ é um vértice de $A_{2}$, temos que $S_{x_{1}} \subset M_{2} \cup N_{2} \cup F_{1, \ell-2}^{\text {forw }} \cup F_{1, \ell-1}^{\text {forw }}$. Portanto,

$$
\mathcal{B}\left(x_{0}\right) \leq d_{F_{1, \ell-2}^{\text {forw }}}\left(x_{0}\right)+d_{F_{1, \ell-2}^{\text {forw }}}\left(x_{0}\right)+d_{M_{2}}\left(x_{0}\right)+d_{N_{2}}\left(x_{0}\right) .
$$

Agora vamos provar que $\mathcal{B}\left(x_{0}\right) \geq d_{F_{1, \ell-2}^{\text {forw }}}\left(x_{0}\right)+d_{F_{1, \ell-1}^{\text {forw }}}\left(x_{0}\right)+d_{M_{2}}\left(x_{0}\right)+d_{N_{2}}\left(x_{0}\right)$. Note que se $x_{0} x_{1}$ é uma aresta de $M_{2} \cup N_{2} \cup F_{1, \ell-2}^{\text {forw }} \cup F_{1, \ell-1}^{\text {forw }}$ que é incidente a $x_{0}$ em $A_{1}$ (essas são as únicas arestas de $G$ que podem contribuir para $\left.\mathcal{B}\left(x_{0}\right)\right)$, então, pela construção de $\mathcal{B}$, existe um rastro $Q^{\prime}=x_{1} \cdots x_{\ell}$ de um caminho tal que o rastro $Q=x_{0} x_{1} \cdots x_{\ell} x_{0}$ (de uma trilha peculiar) pertence a $\mathcal{B}$. Portanto, $\mathcal{B}\left(x_{0}\right)=d_{F_{1, \ell-2}^{\text {forw }}}\left(x_{0}\right)+d_{F_{1, \ell-1}^{\text {forw }}}\left(x_{0}\right)+d_{M_{2}}\left(x_{0}\right)+d_{N_{2}}\left(x_{0}\right)$. Logo, para todo vértice $v \in A_{1}$ temos que

$$
\begin{aligned}
\mathcal{B}(v) & =\left|F_{1, \ell-2}^{\text {forw }}(v)\right|+\left|F_{1, \ell-1}^{\text {forw }}(v)\right|+\left|M_{2}(v)\right|+\left|N_{2}(v)\right| \\
& =2 d^{*}(v)+d_{M_{2}}(v)+d_{N_{2}}(v) \\
& =d_{G_{1}}(v) / \ell+d_{M_{2}}(v)+d_{N_{2}}(v) .
\end{aligned}
$$

Analogamente, temos que $\mathcal{B}(v)=d_{G_{2}}(v) / \ell+d_{M_{1}}(v)+d_{N_{1}}(v)$ para cada vértice $v \in A_{2}$. Logo, $\mathcal{B}$ é uma $\ell$-decomposição $\mathbb{F}$-balanceada.

Afirmação 5.31. $\mathcal{B}$ é $\ell$-pré-completa.

Demonstração. Seja $v \in A_{i}$. Vamos mostrar que $q \operatorname{Pend}(v, \mathcal{B})>\ell$. Note que, pela construção de $\mathcal{B}$, o conjunto de arestas quase-pendentes em $v$ na decomposição $\mathcal{B}$ é exatamente $S_{v}$. Então, $\mathrm{q} \operatorname{Pend}(v, \mathcal{B})=\left|S_{v}\right|=\mathcal{B}^{\prime}(v)$. Como $\mathcal{B}^{\prime}$ é $\mathbb{F}$-balanceada, $\mathcal{B}^{\prime}(v) \geq d_{G_{i}^{\prime}}(v) /(\ell-2)$. Portanto,

$$
\mathrm{qPend}(v, \mathcal{B})=\mathcal{B}^{\prime}(v) \geq d_{G_{i}^{\prime}}(v) /(\ell-2)=2 d^{*}(v)=d_{G_{i}}(v) / \ell
$$

Como $\mathbb{F}$ é uma $(2,2 \ell)$-bifatorização forte de $G$, temos que $d_{G_{i}}(v) \geq \ell(\ell+1)$, de onde concluímos que $\mathrm{qPend}(v, \mathcal{B}) \geq \ell+1$. Portanto, $\mathcal{B}$ é uma $\ell$-decomposição $\ell$-pré-completa.

Agora podemos concluir a prova. Tome $k=\lceil\ell / 2\rceil$ e $r=\lfloor\ell / 2\rfloor$. Note que $\ell=k+r$. Pelo Lema 4.14 com $k$, $\ell$, e $r$, temos que $G$ admite uma $\ell$-decomposição $k$-completa $\mathcal{B}^{\prime \prime}$ tal que $\mathcal{B}(v)=\mathcal{B}^{\prime \prime}(v)$ para todo vértice $v$ de $G$. Como $\mathcal{B}^{\prime \prime}(v)=\mathcal{B}(v)$ para todo vértice $v$ de $G, \mathcal{B}^{\prime \prime}$ é $\mathbb{F}$-balanceada. Pelo Lema 4.16, $G$ admite uma $\ell$-decomposição em caminhos $k$-completa $\mathcal{B}^{*}$ tal que $\mathcal{B}^{*}(v)=\mathcal{B}^{\prime \prime}(v)$ para todo vértice $v$ de $G$. Portanto, $G$ admite uma $\ell$-decomposição em caminhos $\mathbb{F}$-balanceada.

\subsection{Decomposição de grafos altamente aresta-conexos em $\ell$-caminhos}

Nesta seção unimos os resultados da Seção 5.2 com o Teorema 5.27, e provamos a Conjectura 5.2 para caminhos de comprimento fixo. Primeiramente, provamos que a Conjectura 5.2 é equivalente à seguinte conjectura.

Conjectura 5.32. Para cada árvore $T$, existe um inteiro positivo $k_{T}^{\prime \prime}$ tal que, se $G$ é um grafo bipartido $k_{T}^{\prime \prime}$-aresta-conexo e $|E(G)|$ é divisivel por $2|E(T)|$, então $G$ admite uma $T$-decomposição. 
Provamos a Conjectura 5.32 para o caso em que $T$ é um caminho. O resultado a seguir mostra a equivalência da Conjectura 5.32 e a Conjectura 5.2.

Teorema 5.33. Seja $T$ uma árvore com $\ell$ arestas, $\ell \geq 3$, e seja $k$ um inteiro positivo. Se $G$ é um grafo $2(k+\ell)$-aresta-conexo tal que $|E(G)|$ é divisivel por $|E(T)|$, então existe um subgrafo $H$ de $G$ tal que

- $H$ admite uma T-decomposição; $e$

- $G^{\prime}=G-E(H)$ é k-aresta-conexo e $\left|E\left(G^{\prime}\right)\right|$ é divisivel por $2|E(T)|$.

Demonstração. Seja $G$ um grafo $2(k+\ell)$-aresta-conexo tal que $|E(G)|$ é divisível por $|E(T)|$. Se $|E(G)|$ é divisível por $2|E(T)|$, então podemos tomar como $H$ o grafo vazio. Caso contrário, temos que $|E(G)|-\ell$ é divisível por $2|E(T)|$. Nesse caso, seja $H$ uma cópia de $T$ em $G$. Pelo Teorema 5.8, $G$ contém $k+\ell$ árvores geradoras aresta-disjuntas. Como $T$ tem $\ell$ arestas, temos que $H$ intercepta no máximo $\ell$ dessas árvores. Logo, $G-E(H)$ contém pelo menos $k$ árvores geradoras arestas-disjuntas. Portanto, $G-E(H)$ é $k$-aresta-conexo.

Teorema 5.34. Seja $\ell$ um inteiro positivo, e seja $r=\max \{32(\ell-1), \ell(\ell+1)\}$. Se $G$ é um grafo bipartido $(12 \ell+4 r-4)$-aresta-conexo tal que $|E(G)|$ é divisivel por $2 \ell$, então $G$ admite uma $P_{\ell-}$ decomposição.

Demonstração. Sejam $\ell, r$, e $G$ como na hipótese. Pelo Corolário 5.23, $G$ admite uma $(2,2 \ell)$ bifatorização forte $\mathbb{F}$. Pelo Teorema 5.27, $G$ admite uma $\ell$-decomposição em caminhos $\mathbb{F}$-balanceada.

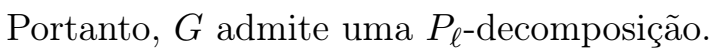

Corolário 5.35. Seja $\ell$ um inteiro positivo, e seja $r=\max \{32(\ell-1), \ell(\ell+1)\}$. Se $G$ é um grafo bipartido $2(13 \ell+4 r-4)$-aresta-conexo tal que $|E(G)|$ é divisivel por $\ell$, então $G$ admite uma $P_{\ell}$-decomposição.

Demonstração. A prova segue aplicando-se o Teorema $5.33 \mathrm{com} k=12 \ell+4 r-r$ e o Teorema 5.34.

Corolário 5.36. Seja $\ell$ um inteiro positivo, $r=\max \{32(\ell-1), \ell(\ell+1)\}$, e tome $k_{T}^{\prime}=2(13 \ell+4 r-4)$. Se $G$ é um grafo $\left(4 k_{T}^{\prime}+16 \ell^{6 \ell+1}\right)$-aresta-conexo tal que $|E(G)|$ é divisivel por $\ell$, então $G$ admite uma $P_{\ell}$-decomposição.

Demonstração. A prova segue diretamente do Corolário 5.35 e do Teorema 5.3. 


\section{Capítulo 6}

\section{Considerações finais}

Decomposição de grafos é um tópico que tem se mostrado rico em conjecturas e problemas desafiadores que têm trazido contribuições relevantes na área de teoria estrutural de grafos. Neste trabalho desenvolvemos uma técnica para tratar decomposições de grafos em caminhos que se mostrou frutífera para atacar problemas atuais e muito estudados (as Conjecturas 1.1, 4.1, e 5.1). Além disso, as ferramentas desenvolvidas neste trabalho têm levado à obtenção de novos resultados, como em [BT], desenvolvido durante a elaboração deste texto.

Ressaltamos que este trabalho se beneficiou bastante dos resultados de Thomassen para decomposições de grafos altamente aresta-conexos; esperamos que essa conexão tenha ficado clara ao leitor familiarizado com esses resultados. Também gostaríamos de mencionar que o resultado de Merker [Mer15] contribui para a literatura como uma alternativa aos resultados de fatorização e bifatorização apresentados neste trabalho. Em particular, se for possível generalizar o Lema de Desemaranhamento para lidar com árvores mais gerais, o resultado de Merker pode ser aplicado para resolver a Conjectura 5.1 para tais árvores.

Enquanto escrevíamos o resultado principal do Capítulo 5 em [BMOWb], fomos informados que Bensmail, Harutyunyan, Le, e Thomassé [BHLT15] obtiveram um resultado similar usando uma abordagem diferente. Isso deixa claro que o estudo de decomposições de grafos permite explorar técnicas bastante diversas da teoria de grafos. Para ilustrar um pouco mais tal fato, recomendamos a leitura de $\left[\mathrm{CKL}^{+} 13\right.$, GKO15].

Pretendemos continuar trabalhando nas Conjecturas 1.1 e 5.1, com o objetivo de estender a família de árvores para as quais elas são válidas. Seria bastante interessante generalizar o Lema de Desemaranhamento para lidar com estruturas mais gerais, pois, como observado acima, isso implicaria numa prova construtiva da Conjectura 5.1. Além disso, uma tal generalização nos permitiria obter resultados análogos aos da Seção 4 para classes mais gerais de árvores.

Em outra direção, acreditamos que podemos melhorar a condição sobre a cintura do grafo no caso da Conjectura 4.1. 


\section{Referências Bibliográficas}

[AK11] Jin Akiyama e Mikio Kano. Factors and factorizations of graphs, volume 2031 of Lecture Notes in Mathematics. Springer, Heidelberg, 2011. Proof techniques in factor theory. 35

[BF83] André Bouchet e Jean-Luc Fouquet. Trois types de décompositions d'un graphe en chaînes. Em Combinatorial mathematics (Marseille-Luminy, 1981), volume 75 of NorthHolland Math. Stud., páginas 131-141. North-Holland, Amsterdam, 1983. 19, 27

[BG14] János Barát e Dániel Gerbner. Edge-decompositions of graphs into copies of a tree with four edges. Electron. J. Combin., 21(1):Research Paper 55 pp. (electronic), 2014. 31, 35

[BHLT15] J. Bensmail, A. Harutyunyan, T.-N. Le e S. Thomassé. Edge-partitioning a graph into paths: beyond the Barát-Thomassen conjecture. ArXiv e-prints, Julho 2015. 31, 45

[BJ] Fábio Botler e Andrea Jiménez. On path decompositions of 2k-regular graphs. Submetido. [Resumo estendido: On path decompositions of 2k-regular graphs. Electronic Notes in Discrete Mathematics, vol. 50 pp 163-168, 2015]. 20

[BKLO16] Ben Barber, Daniela Kühn, Allan Lo e Deryk Osthus. Edge-decompositions of graphs with high minimum degree. Advances in Mathematics, 288:337 - 385, 2016. 1

[BMOWa] Fábio Botler, Guilherme O. Mota, Marcio T. I. Oshiro e Yoshiko Wakabayashi. Decomposing highly connected graphs into paths of length five. Submetido. [Resumo estendido: Decompositions of highly connected graphs into paths of length five. Electronic Notes in Discrete Mathematics, vol. 50, pp 211-216, 2015]. 31, 36

[BMOWb] Fábio Botler, Guilherme O. Mota, Marcio T. I. Oshiro e Yoshiko Wakabayashi. Decomposing highly edge-connected graphs into paths of any given length. Submetido. [Resumo estendido: Decompositions of highly connected graphs into paths of any given length. Electronic Notes in Discrete Mathematics, vol 49, pp 795-802, 2015]. 3, 7, 29, 31,45

[BMOWc] Fábio Botler, Guilherme O. Mota, Marcio T. I. Oshiro e Yoshiko Wakabayashi. Decomposing regular graphs with prescribed girth into paths. Em preparação. [Resumo estendido: Path decompositions of regular graphs with prescribed girth. Electronic Notes in Discrete Mathematics, vol. 49, pp 629-636, 2015]. 3

[BMW15] Fábio Botler, Guilherme O. Mota e Yoshiko Wakabayashi. Decompositions of trianglefree 5-regular graphs into paths of length five. Discrete Math., 338(11):1845-1855, 2015. $3,7,20,22,26$

[Bol98] Béla Bollobás. Modern graph theory, volume 184 of Graduate Texts in Mathematics. Springer-Verlag, New York, 1998. 5

[Bon14] Adrian Bondy. Beautiful conjectures in graph theory. European J. Combin., 37:4-23, 2014. 20 
[BT] Fábio Botler e Alexandre Talon. Decomposing regular graphs into paths of length four. Em preparação. 21, 45

[BT06] János Barát e Carsten Thomassen. Claw-decompositions and Tutte-orientations. J. Graph Theory, 52(2):135-146, 2006. 2, 31

$\left[\mathrm{CKL}^{+} 13\right]$ Béla Csaba, Daniela Kühn, Allan Lo, Deryk Osthus e Andrew Treglown. Proof of the 1-factorization and Hamilton decomposition conjectures. Em The Seventh European Conference on Combinatorics, Graph Theory and Applications, volume 16 of CRM Series, páginas 477-482. Ed. Norm., Pisa, 2013. 45

[Die10] Reinhard Diestel. Graph theory, volume 173 of Graduate Texts in Mathematics. Springer, Heidelberg, fourth edição, 2010. 5

[DT97] Dorit Dor e Michael Tarsi. Graph decomposition is NP-complete: a complete proof of Holyer's conjecture. SIAM J. Comput., 26(4):1166-1187, 1997. 1

[EH06] Michelle Edwards e Lea Howard. A survey of graceful trees. Atl. Electron. J. Math., 1(1):5-30, 2006. 2

[ENV02] M. N. Ellingham, Yunsun Nam e Heinz-Jürgen Voss. Connected $(g, f)$-factors. J. Graph Theory, 39(1):62-75, 2002. 35

[Erd14] Joshua Erde. Decomposing the cube into paths. Discrete Math., 336:41-45, 2014. 2

[FGK10] Odile Favaron, François Genest e Mekkia Kouider. Regular path decompositions of odd regular graphs. J. Graph Theory, 63(2):114-128, 2010. 2, 19

[Fin90] John Frederick Fink. On the decomposition of $n$-cubes into isomorphic trees. J. Graph Theory, 14(4):405-411, 1990. 2

[GKO15] S. Glock, D. Kühn e D. Osthus. Optimal path and cycle decompositions of dense quasirandom graphs. ArXiv e-prints, Março 2015. 45

[Gol72] Solomon W. Golomb. How to number a graph. Em Graph theory and computing, páginas 23-37. Academic Press, New York, 1972. 2

[Häg89] Roland Häggkvist. Decompositions of complete bipartite graphs. Em Surveys in combinatorics, 1989 (Norwich, 1989), volume 141 of London Math. Soc. Lecture Note Ser., páginas 115-147. Cambridge Univ. Press, Cambridge, 1989. 1, 2

[HLY99] Katherine Heinrich, Jiping Liu e Minli Yu. $P_{4}$-decompositions of regular graphs. $J$. Graph Theory, 31(2):135-143, 1999. 7, 21, 24

[Jae88] François Jaeger. Nowhere-zero flow problems. Em Selected topics in graph theory, 3, páginas 71-95. Academic Press, San Diego, CA, 1988. 31

[JKW13] Kyle F. Jao, Alexandr V. Kostochka e Douglas B. West. Decomposition of Cartesian products of regular graphs into isomorphic trees. J. Comb., 4(4):469-490, 2013. 2

[JRP85] M. Jünger, G. Reinelt e W. R. Pulleyblank. On partitioning the edges of graphs into connected subgraphs. J. Graph Theory, 9(4):539-549, 1985. 1

[JTT91] Michael S. Jacobson, Mirosław Truszczyński e Zsolt Tuza. Decompositions of regular bipartite graphs. Discrete Math., 89(1):17-27, 1991. 2

[KL99] Mekkia Kouider e Zbigniew Lonc. Path decompositions and perfect path double covers. Australas. J. Combin., 19:261-274, 1999. 2, 7, 21, 22, 26 
[Kot57] Anton Kotzig. Aus der Theorie der endlichen regulären Graphen dritten und vierten Grades. Časopis Pěst. Mat., 82:76-92, 1957. 2, 19, 27

[Lov68] L. Lovász. On covering of graphs. Em Theory of Graphs (Proc. Colloq., Tihany, 1966), páginas 231-236. Academic Press, New York, 1968. 20

[LTWZ13] László Miklós Lovász, Carsten Thomassen, Yezhou Wu e Cun-Quan Zhang. Nowherezero 3-flows and modulo k-orientations. J. Combin. Theory Ser. B, 103(5):587-598, 2013. 34

[Mad78] W. Mader. A reduction method for edge-connectivity in graphs. Ann. Discrete Math., 3:145-164, 1978. Advances in graph theory (Cambridge Combinatorial Conf., Trinity College, Cambridge, 1977). 33

[Men27] Karl Menger. Zur allgemeinen Kurventheorie. Fundamenta Mathematicae, 10(1):96-115, 1927. 6

[Mer15] Martin Merker. Decomposition of graphs. Tese de Doutorado, Technical University of Denmark, 2015. Em preparação. 31, 45

[NW61] C. St. J. A. Nash-Williams. Edge-disjoint spanning trees of finite graphs. J. London Math. Soc., 36:445-450, 1961. 34

[NW70] C. St. J. A. Nash-Williams. An unsolved problem concerning decomposition of graphs into triangles. Combinatorial Theory and its Applications III, páginas 1179-1183, 1970. 1

[NW85] C. St. J. A. Nash-Williams. Connected detachments of graphs and generalized Euler trails. J. London Math. Soc. (2), 31(1):17-29, 1985. 33

[Pet91] Julius Petersen. Die Theorie der regulären graphs. Acta Math., 15(1):193-220, 1891. 6, 35

[Pyb92] L. Pyber. Covering the edges of a graph by .... Em Sets, graphs and numbers (Budapest, 1991), volume 60 of Colloq. Math. Soc. János Bolyai, páginas 583-610. North-Holland, Amsterdam, 1992. 1

[Rin64] G. Ringel. Problem n.25. Em Theory of Graphs and its Applications (Proc. Sympos. Smolenice, 1963). Publ. House Czechoslovak Acad. Sci., Prague, 1964. 2

[Ros67] A. Rosa. On certain valuations of the vertices of a graph. Em Theory of Graphs (Internat. Sympos., Rome, 1966), páginas 349-355. Gordon and Breach, New York; Dunod, Paris, 1967. 2

[Sne91] Hunter Saint Clair Snevily. Combinatorics of finite sets. ProQuest LLC, Ann Arbor, MI, 1991. Thesis (Ph.D.)-University of Illinois at Urbana-Champaign. 2

[Tho08a] Carsten Thomassen. Decompositions of highly connected graphs into paths of length 3. J. Graph Theory, 58(4):286-292, 2008. 2, 31, 36, 40

[Tho08b] Carsten Thomassen. Edge-decompositions of highly connected graphs into paths. Abh. Math. Semin. Univ. Hambg., 78(1):17-26, 2008. 2, 31

[Tho12] Carsten Thomassen. The weak 3-flow conjecture and the weak circular flow conjecture. J. Combin. Theory Ser. B, 102(2):521-529, 2012. 2, 31, 34

[Tho13a] Carsten Thomassen. Decomposing a graph into bistars. J. Combin. Theory Ser. B, 103(4):504-508, 2013. 2, 31, 34 
[Tho13b] Carsten Thomassen. Decomposing graphs into paths of fixed length. Combinatorica, 33(1):97-123, 2013. 2, 31

[Tut61] W. T. Tutte. On the problem of decomposing a graph into $n$ connected factors. $J$. London Math. Soc., 36:221-230, 1961. 34

[VB37] F. Von Baebler. Über die Zerlegung regulärer Streckenkomplexe ungerader Ordnung. Comment. Math. Helv., 10(1):275-287, 1937. 35 